CIVIL ENGINEERING STUDIES

Illinois Center for Transportation Series No. 20-003

UILU-ENG-2020-2003

ISSN: 0197-9191

\title{
Size and Shape Determination of Riprap and Large-sized Aggregates Using Field Imaging
}

\author{
Prepared by \\ Haohang Huang \\ Jiayi Luo \\ Erol Tutumluer, PhD \\ John M. Hart \\ Issam Qamhia, PhD \\ University of Illinois at Urbana-Champaign
}

Research Report No. FHWA-ICT-20-002

A report of the findings of

ICT PROJECT R27-182

Size and Shape Determination of Riprap and Large-sized

Aggregates Using Field Imaging

https://doi.org/10.36501/0197-9191/20-003

Illinois Center for Transportation

January 2020 

TECHNICAL REPORT DOCUMENTATION PAGE

\begin{tabular}{|l|l|}
\hline 1. Report No. & 2. Government Accession No. \\
FHWA-ICT-20-002 & N/A \\
\hline
\end{tabular}

\section{Title and Subtitle}

Size and Shape Determination of Riprap and Large-sized Aggregates Using Field Imaging

\section{Recipient's Catalog No.} N/A

\section{Report Date}

January 2020

6. Performing Organization Code N/A

8. Performing Organization Report No.

ICT 20-003

UILU-ENG-2020-2003

\section{Work Unit No.}

$\mathrm{N} / \mathrm{A}$

11. Contract or Grant No.

R27-182

205 North Mathews Avenue, MC-250

Urbana, IL 61801

\section{Sponsoring Agency Name and Address}

Illinois Department of Transportation (SPR)

Bureau of Research

126 East Ash Street

Springfield, IL 62704

\section{Supplementary Notes}

Conducted in cooperation with the U.S. Department of Transportation, Federal Highway Administration.

https://doi.org/10.36501/0197-9191/20-003

\section{Abstract}

Riprap rock and large-sized aggregates are extensively used in transportation, geotechnical, and hydraulic engineering applications. Traditional methods for assessing riprap categories based on particle weight may involve subjective visual inspection and time-consuming manual measurements. Aggregate imaging and segmentation techniques can efficiently characterize riprap particles for their size and morphological/shape properties to estimate particle weights. Particle size and morphological/shape characterization ensure the reliable and sustainable use of all aggregate skeleton materials at quarry production lines and construction sites. Aggregate imaging systems developed to date for size and shape characterization, however, have primarily focused on measurement of separated or non-overlapping aggregate particles. This research study presents an innovative approach for automated segmentation and morphological analyses of stockpile aggregate images based on deep-learning techniques. As a project outcome, a portable, deployable, and affordable field-imaging system is envisioned to estimate volumes of individual riprap rocks for field evaluation. A state-of-the-art object detection and segmentation framework is used to train an image-segmentation kernel from manually labeled 2D riprap images in order to facilitate automatic and userindependent segmentation of stockpile aggregate images. The segmentation results show good agreement with ground-truth validation, which entailed comparing the manual labeling to the automatically segmented images. A significant improvement to the efficiency of size and morphological analyses conducted on densely stacked and overlapping particle images is achieved. The algorithms are integrated into a software application with a user-friendly Graphical User Interface (GUI) for ease of operation. Based on the findings of this study, this stockpile aggregate image analysis program promises to become an efficient and innovative application for field-scale and in-place evaluations of aggregate materials. The innovative imaging-based system is envisioned to provide convenient, reliable, and sustainable solutions for the on-site quality assurance/quality control (QA/QC) tasks related to riprap rock and large-sized aggregate material characterization and classification.

\section{Key Words \\ 18. Distribution Statement}

Riprap, Large-sized Aggregates, Field Imaging, Computer Vision, Deep Learning, Object Detection, Image Segmentation, Size and Morphological/Shape Analyses, Stockpile Aggregate Imaging

No restrictions. This document is available through the National Technical Information Service, Springfield, VA 22161.
19. Security Classif. (of this report)
Unclassified
20. Security Classif. (of this page)
Unclassified
21. No. of Pages
22. Price 68 $\mathrm{N} / \mathrm{A}$

Form DOT F 1700.7 (8-72)

Reproduction of completed page authorized 



\section{ACKNOWLEDGMENT, DISCLAIMER, MANUFACTURERS' NAMES}

This publication is based on the results of "ICT-R27-182: Size and Shape Determination of Riprap and Large-sized Aggregates Using Field Imaging." ICT-R27-182 was conducted in cooperation with the Illinois Center for Transportation; the Illinois Department of Transportation; and the U.S. Department of Transportation, Federal Highway Administration.

Members of the Technical Review Panel (TRP) were the following:

- Andrew Stolba, TRP Chair, Illinois Department of Transportation

- Sheila Beshears, Riverstone Group, Inc.; Former TRP Chair with the Illinois Department of Transportation

- Chad Nelson, Illinois Department of Transportation-District 3

- Del Reeves, Illinois Department of Transportation-District 6

- Kevin Tressel, Illinois Department of Transportation

- Megan Swanson, Illinois Department of Transportation

- Ryan Culton, Formerly with Illinois Department of Transportation

- Sean Stutler, Illinois Department of Transportation

- Dennis Bachman, Federal Highway Administration

- Andrew Buck, Vulcan Materials Company

- Dan Barnstable, Vulcan Materials Company

- Jim Sergent, Lehigh Hanson

The authors would like to extend their appreciation to the current and past TRP Chairs and all TRP members for their time and significant contributions to this research project. The help of Andrew Stolba, Sheila Beshears, Chad Nelson, Andrew Buck, and Dan Barnstable in coordinating quarry field visits to sample and image individual riprap rocks and stockpiles is highly appreciated.

The authors would like to express their sincere gratitude to the University of Illinois at UrbanaChampaign (UIUC) and Illinois Center for Transportation (ICT) students who helped on several tasks dealing with materials characterization, testing, image acquisition, and manual labeling of rocks in captured stockpile image datasets, namely: Zixu Zhao, Han Wang, Jiawei Fan, and Zhongyi Liu. Sincere acknowledgements are due to Prof. Maziar Moaveni (Savannah State University, formerly with the University of Illinois) and Huseyin Boler for their insightful advice to the project.

The authors would like to acknowledge the technical support received from the Computer Vision and Robotics Laboratory in the Beckman Institute for Advanced Science and Technology at UIUC. Sincere acknowledgments are also due to ICT research engineer Greg Renshaw for his support throughout the project. 
The authors are particularly grateful for the help with material procurement and technical support given by Timothy Prunkard, Megan Everette, and Suzanne Lowry from the Department of Civil and Environmental Engineering at UIUC. A great thank you is extended to Kristi Anderson, Audrey Donoho, McCall Macomber, and Brian Lorbiecki for ICT project management.

The contents of this report reflect the views of the authors, who are responsible for the facts and the accuracy of the data presented herein. The contents do not necessarily reflect the official views or policies of the Illinois Center for Transportation, the Illinois Department of Transportation, or the Federal Highway Administration. This report does not constitute a standard, specification, or regulation.

Trademark or manufacturers' names appear in this report only because they are considered essential to the object of this document and do not constitute an endorsement of product by the Federal Highway Administration, the Illinois Department of Transportation, or the Illinois Center for Transportation. 


\section{EXECUTIVE SUMMARY}

This research project provides a solution for automating the process of size/shape characterization and classification of riprap and large-sized aggregates during on-site quality assurance/quality control (QA/QC) evaluations. Riprap is rock or other material used to armor shorelines, streambeds, bridge abutments, pilings, and other shoreline structures against scour and water or ice erosion. It is made from a variety of rock types, commonly granite or limestone and occasionally concrete rubble from building and paving demolition. Determination of particle-size distribution or gradation of riprap has always been difficult. The riprap gradation or sizing of the pieces shall be well graded, as outlined in Illinois Department of Transportation (IDOT) specifications. Individual rock pieces are measured for weight, which is a time-consuming and labor-intensive task. In this regard, reliable field-imaging techniques are needed to easily and quickly process stockpile images of riprap for gradation checks and provide actionable data analytics. Over the past two decades, imaging-based analysis techniques have been widely developed and adopted to characterize aggregate particle size and shape properties with high accuracy and efficiency.

This research effort, conducted at the Illinois Center for Transportation (ICT), focused on developing a convenient, nonintrusive field-evaluation system based on imaging. An engineer or inspector can take multiple photos/images of the riprap and large-sized aggregates for the gradation (size distribution) and shape property determination tasks with a field imaging and evaluation kit. The final fieldimaging system was developed to be portable, affordable, and accurate for reliable data compilation under field conditions. The specific goals of the research study were as follows:

- Identify aggregate producers of riprap and large-sized aggregates in Illinois, and acquire image data and representative samples from field visits. Gather information on the types, geological origins, and sources of the materials. Study applicable current manual methods for QA/QC.

- Develop field-imaging algorithms that accomplish user-independent segmentation for robust operation under a wide variety of riprap stockpile field conditions, e.g., natural sunlight and/or shadow considerations.

- Develop software algorithms for size and shape analyses of individual aggregate particles captured from multiple views, and integrate the algorithms into a software application with Graphical User Interface (GUI).

First, a review of riprap $Q A / Q C$ requirements and previous research studies was conducted, including aggregate imaging techniques and developed systems and emerging artificial intelligence-based approaches. The review identified that the state of the practice for assessing riprap geometric properties involves subjective visual inspection and time-consuming hand measurements, whereas achieving the comprehensive in situ characterization of riprap materials remains challenging for practitioners and engineers. Although several advanced aggregate imaging systems utilized computer vision techniques for size and shape characterization, these systems developed to date have primarily focused on measurement of separated or non-overlapping aggregate particles. Image-based evaluations of densely stacked (or stockpile) aggregates require image segmentation of a stockpile to determine the size and morphological properties of individual particles. To accomplish this goal, there 
is an urgent need to develop efficient computer vision algorithms based on traditional computer vision techniques and/or emerging deep-learning techniques.

Riprap and large-sized aggregate samples were collected from quarries, and images of single rocks were acquired first from designated size riprap categories. IDOT size categories for riprap and lists of approved riprap sources were identified, and a series of field visits to different aggregate producer sites were scheduled. The development of a riprap inspection system to image individual rocks as well as riprap stockpiles was considered next. Both laboratory and field measurements provided ground-truth data for validating the imaging system. Images acquired from the field visits were then used in a two-phase research approach: (1) a single-particle imaging study that focused on volumetric reconstruction of individual riprap rocks and (2) a riprap stockpile imaging study that accomplished the task of stockpile image segmentation and morphological analysis.

For the single-particle imaging study, the volumetric properties of riprap rocks were established from a field-imaging system associated with newly developed color image segmentation and volume estimation algorithms. This system consists of a field imaging and evaluation kit whereby an engineer or inspector can take multiple photos under field lighting conditions. An associated imagesegmentation algorithm was developed with the capability to extract object information with shadow effects. Based on multi-view information from the image-segmentation algorithm, the volumetric properties can be estimated. Both algorithms are user-independent with minimal user input required during image-processing stages for ease in implementation and practical use. The field-imaging methods have been tested and evaluated to be portable, deployable, and affordable for efficient image acquisition. The robustness and accuracy of the image segmentation and volume estimation were validated against ground-truth measurements collected at stone quarry sites and compared with state-of-the-practice inspection methods for single-particle volumetric estimation.

For the riprap stockpile imaging study, an innovative approach for automated segmentation and morphological analyses of stockpile aggregate images based on deep-learning techniques was established. A task-specific stockpile aggregate image dataset was compiled of images collected from quarries in Illinois. Individual particles in the stockpile images were manually labeled on each image. A state-of-the-art object detection and segmentation framework called Mask R-CNN (RegionalConvolutional Neural Network) was then implemented to train the image-segmentation kernel to enable segmentation of stockpile aggregate images. The segmentation results showed good agreement with ground-truth labeling and improved the efficiency of size and morphological analyses conducted on images containing densely stacked and overlapping particles.

As the key deliverable, a practical, user-independent, and user-friendly software application was developed in this research study. The software application was developed to identify riprap or largesized aggregates for evaluation of size/shape of individual aggregate particles captured by the fieldimaging kit. Professional software engineering guidelines were reviewed and strictly followed during the developmental stages. The riprap stockpile image analysis algorithms were programmed and integrated into a standalone software application with an easy-to-use user interface. A user manual is provided along with this final report for the developed software program. 
In conclusion, imaging systems for both single-rock riprap and stockpiles were developed, validated, and integrated into a software application with GUI. The single-particle imaging system accomplished the tasks of image segmentation and volumetric reconstruction for inspecting individual riprap rocks. The riprap stockpile imaging system tackled the tasks of dense image segmentation and morphological analyses for the evaluation of riprap stockpiles. Both systems have demonstrated good agreement with ground-truth data and showed future potential as state-of-the-art size and shape characterization techniques for riprap and large-sized aggregates. The findings of this study also indicated a promising future research and development focus on leveraging advanced 3D reconstruction technology based on the point cloud segmentation of stockpile images. 


\section{TABLE OF CONTENTS}

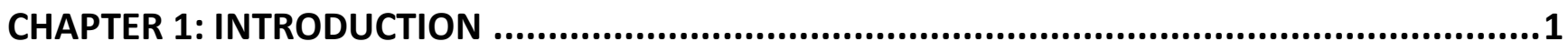

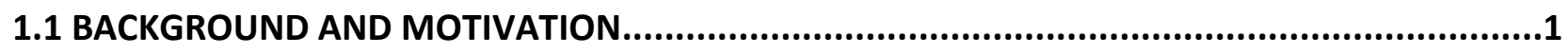

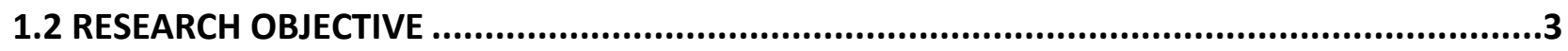

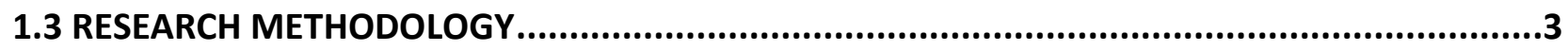

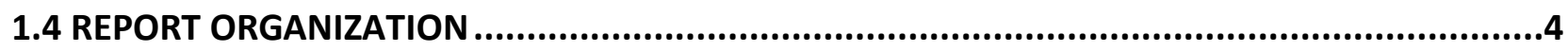

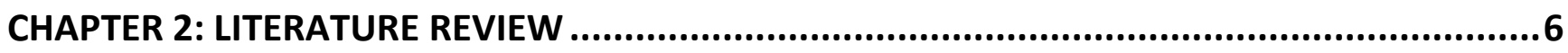

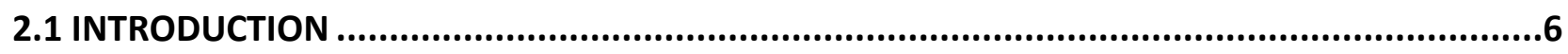

2.2 APPLICATIONS AND SPECIFICATIONS OF RIPRAP AND LARGE-SIZED AGGREGATES ...............6

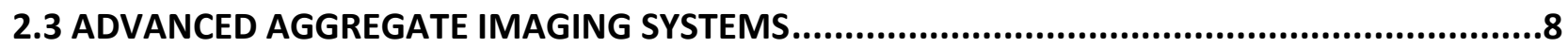

2.4 IMAGE-PROCESSING APPROACHES FOR AGGREGATE EVALUATION ................................9

2.5 MACHINE-LEARNING FACILITATED COMPUTER-VISION TECHNIQUES...............................10

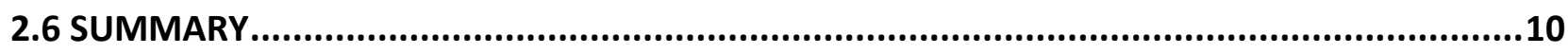

CHAPTER 3: FIELD STUDIES AND SAMPLING OF RIPRAP MATERIAL IN QUARRIES................12

3.1 INTRODUCTION .................................................................................................................12

3.2 OVERVIEW OF IDOT RIPRAP SIZE AND SHAPE REQUIREMENTS ...................................12

3.3 SELECTION OF RIPRAP MATERIAL AND AGGREGATE PRODUCERS ...................................14

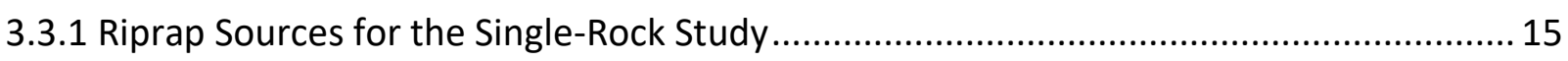

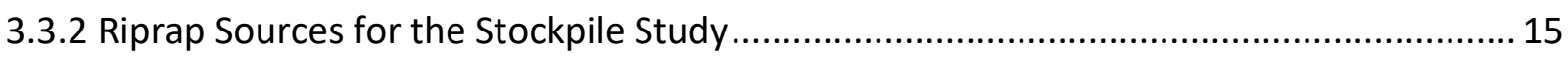

3.4 RIPRAP IMAGE DATA COLLECTION .................................................................16

3.4.1 Single-Rock Image Acquisition, Sampling, and Measurement Procedure...................... 17

3.4.2 Riprap Stockpile Image-acquisition Procedure ....................................................... 18

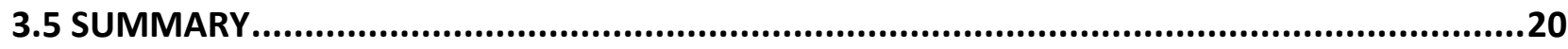

CHAPTER 4: SINGLE-PARTICLE VOLUMETRIC ESTIMATION USING COMPUTER VISION........21

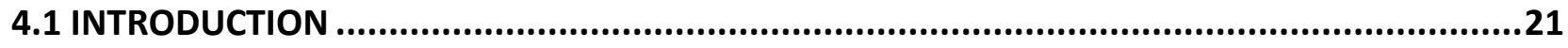

4.2 COLOR-BASED IMAGE-SEGMENTATION ALGORITHM FOR OBJECT DETECTION .................21

4.2.1 Color Representation Using CIE L*a*b* Space ................................................... 21

4.2.2 Foreground and Background Representation Using Pixel Statistics.............................. 23

4.2.3 Contrast Enhancement Based on Color Distance .................................................... 24

4.2.4 Adaptive Thresholding and Morphological De-noising ........................................... 25 
4.3 3D VOLUMETRIC RECONSTRUCTION ALGORITHM ..................................................26

4.3.1 Image Resizing Based on Calibration Ball Reference ................................................... 26

4.3.2 Orthogonality Calibration Using Least Squares Solution ........................................... 27

4.3.3 Spatial Intersection of Multi-View Silhouettes ...................................................... 28

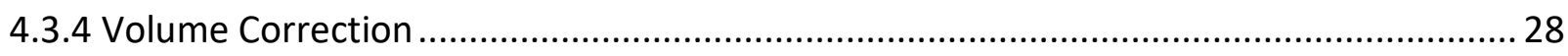

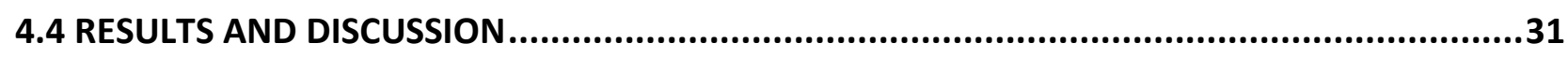

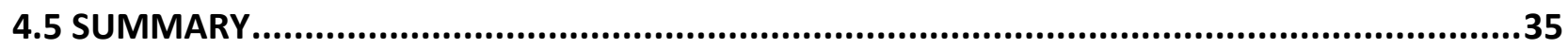

CHAPTER 5: RIPRAP STOCKPILE PARTICLE SIZE AND SHAPE ESTIMATION USING DEEP

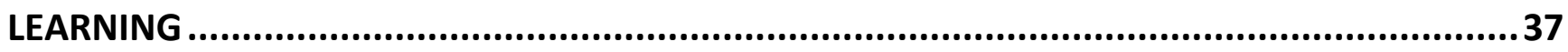

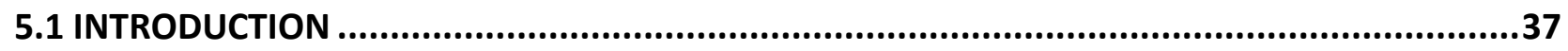

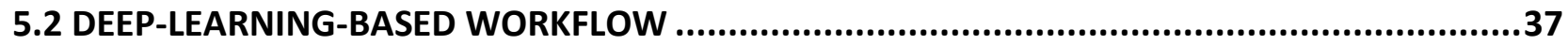

5.3 LABELED DATASET OF RIPRAP STOCKPILE IMAGES................................................38

5.4 DEEP-LEARNING FRAMEWORK FOR AUTOMATED IMAGE SEGMENTATION......................40

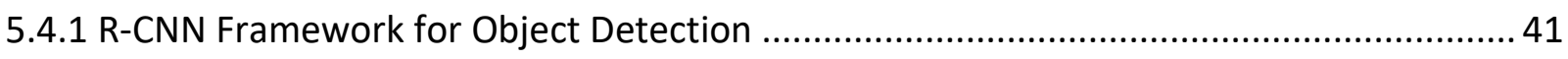

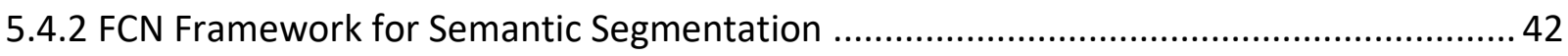

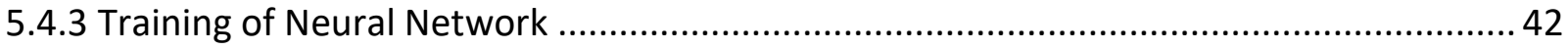

5.5 MORPHOLOGICAL ANALYSIS ALGORITHMS OF STOCKPILE PARTICLES ............................43

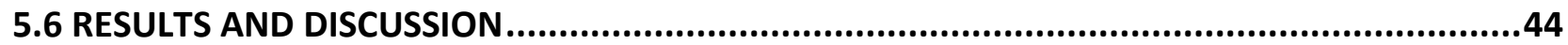

5.6.1 Comparison of Image-Segmentation Results ..................................................... 44

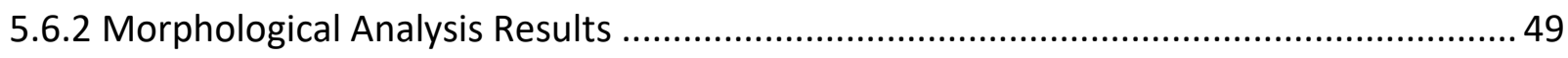

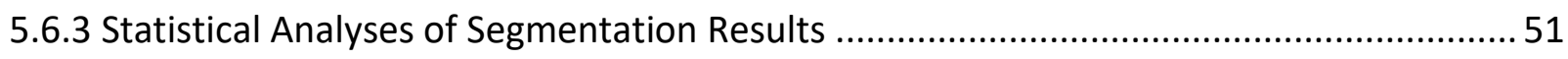

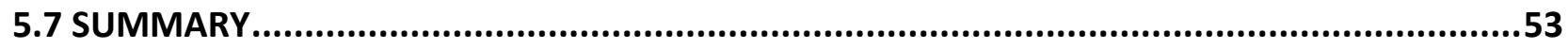

CHAPTER 6: SOFTWARE DEVELOPMENT FOR RIPRAP STOCKPILE CHARACTERIZATION........54

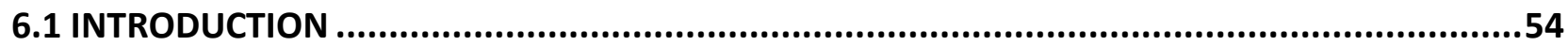

6.2 REVIEW OF SOFTWARE ENGINEERING CONCEPTS..................................................54

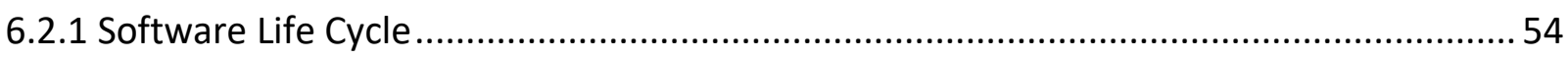

6.2.2 Lifecycle Model of Software Development................................................................. 54

6.3 DESIGN AND CONTROL LOGIC OF GRAPHICAL USER INTERFACE (GUI) .............................55

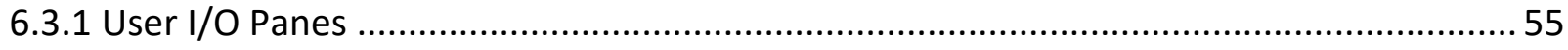

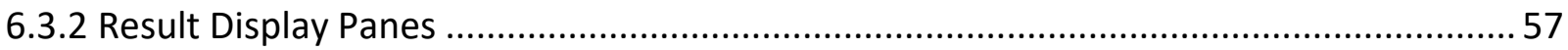

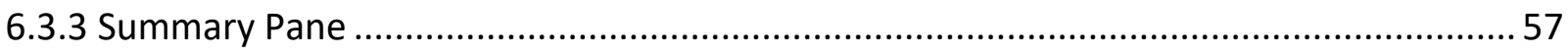

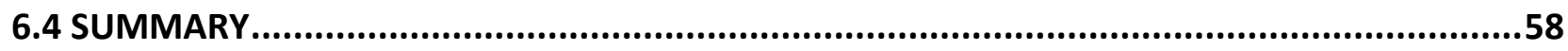




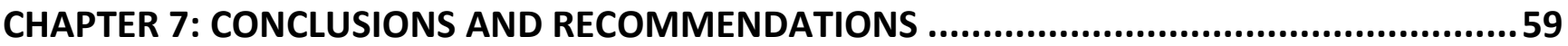

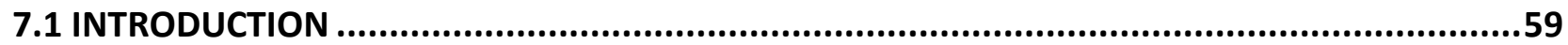

7.2 SUMMARY OF FINDINGS FROM THE SINGLE-PARTICLE STUDY .....................................59

7.3 SUMMARY OF FINDINGS FROM THE RIPRAP STOCKPILE STUDY .....................................60

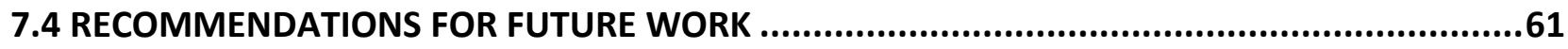

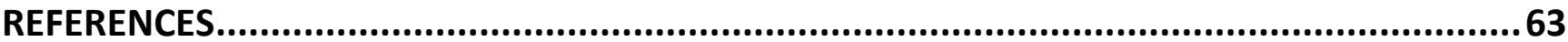




\section{LIST OF FIGURES}

Figure 3.1. Converted particle-size distribution of IDOT riprap categories RR3-RR7.

Figure 3.2. Geographical information for selected IDOT-approved aggregate producers (IDOT's Approved/Qualified Producer List of Aggregate Sources 2018).

Figure 3.3. Geographical information for the aggregate producers that participated in this study. .... 16

Figure 3.4. (a) A conceptual illustration and (b) the actual constructed setup of the field-imaging system for single rocks.

Figure 3.5. (a) Hand measurements of riprap dimensions and (b) tripod scale system used by IDOT for measuring the weight of large riprap rocks.

Figure 3.6. (a) Proper positioning of the camera relative to the stockpile, (b) satisfactory image with a calibration ball, (c) satisfactory image without a calibration ball, and (d) unsatisfactory image of a stockpile.

Figure 4.1. Schema of (a) RGB color space, (b) HSV color space, and (c) CIE L*a*b* color space (Kothari 2018).

Figure 4.2. Channel images of a large riprap rock studied using CIE L*a*b* color space.

Figure 4.3. (a) Pixel histogram of color channel and (b) pixel cumulative distribution function of color channel.

Figure 4.4. (a) Original b* channel image and (b) distance map with foreground representative color as the reference.

Figure 4.5. (a) Adaptive thresholding applied image, (b) morphological de-noising applied image, and (c) image-segmentation result.

Figure 4.6. Flowchart of 3D volumetric reconstruction algorithm.

Figure 4.7. Pixel-scope difference between the detected boundary and actual boundary..... 29

Figure 4.8. Effect of rock/ball ratio and calibration ball size on volume overestimation.

Figure 4.9. Comparisons between image analysis results and ground-truth measurements for:

(a) Source 1 material with rotate repetitions, (b) Source 1 material after averaging, (c) Source 2 material with rotate repetitions, (d) Source 2 material after averaging, (e) all sources with rotate repetitions, and $(\mathrm{f})$ all sources after averaging $(1 \mathrm{lb} .=0.454 \mathrm{~kg})$

Figure 4.10. Comparisons between weights estimated from image analyses and weights estimated from hand measurements on Source 2 and Source 2-Large particles (1 lb. $=0.454 \mathrm{~kg}$ ).

Figure 5.1. Flowchart of deep-learning-based image segmentation and morphological analysis approach.

Figure 5.2. Stockpile aggregate image (a) before labeling and (b) after labeling.

Figure 5.3. Model architecture of the Mask R-CNN framework composed of (a) Region-based Convolutional Neural Network (R-CNN) and (b) Fully Convolutional Network (FCN)..... 
Figure 5.4. Training loss of (a) entire Mask R-CNN network, (b) R-CNN object localization network, (c) R-CNN object classification network, and (d) FCN semantic segmentation network.

Figure 5.5. Raw images of (a) separated particles, (b) contacting or overlapping particles, and (c) densely stacked particles. Watershed segmented images of (d) separated particles, (e) contacting or overlapping particles, and (f) densely stacked particles. Mask R-CNN segmented images of (g) separated particles, (h) contacting or overlapping particles, and (i) densely stacked particles....... 45

Figure 5.6. Additional stockpile image-segmentation results.................................................... 48

Figure 5.7. (a) Mask R-CNN segmented image (enlarged from Figure 4[i]); (b) histogram distribution and (c) cumulative distribution curves for equivalent particle size; and (d) histogram distribution and (e) cumulative distribution curves for flat and elongated ratio. 50

Figure 5.8. Completeness and precision metrics used to compare the segmentation results with the ground-truth labeling 51

Figure 6.1. Schematic drawing of Graphical User Interface (GUI) control logic. 56

Figure 6.2. Screenshot of graphical user interface. .58 


\section{LIST OF TABLES}

Table 3.1. Keystone Requirements for Different Riprap Size/Weight Categories ............................. 12

Table 3.2. Grid Size Requirements for Sampling Different Riprap Gradation Categories .................... 13

Table 3.3. Material Size and Source Information for the Single-Rock Study .................................. 15

Table 3.4. Source Information and Description of Stockpile Aggregate Image Dataset ..................... 16

Table 4.1. Comparisons between Image Analysis Results and Hand Measurements on Source 2

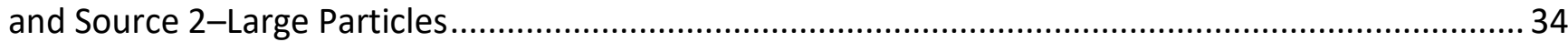

Table 5.1. Source Information and Description of Stockpile Aggregate Image Dataset ...................... 39

Table 5.2. Completeness and Precision Results of Randomly Selected Validation Set Images ............. 52 



\section{CHAPTER 1: INTRODUCTION}

\subsection{BACKGROUND AND MOTIVATION}

Riprap is rock or other material used to armor shorelines, streambeds, bridge abutments, pilings, and other shoreline structures against scour and water or ice erosion. It is made from a variety of rock types, commonly granite or limestone and occasionally concrete rubble from building and paving demolition. Determination of particle-size distribution or gradation of riprap has always been challenging. The riprap gradation or sizing of the pieces shall be well-graded, as outlined in Article 1005.01(c) of IDOT's Standard Specifications for Road and Bridge Construction (IDOT 2016). Riprap rock pieces are often weighed individually to fit into seven riprap weight categories (RR1 to RR7), which is a time-consuming and labor-intensive task that is often hard to calibrate with inspectors. Sample grid systems have been used for gradations of RR3 to RR7. Recently, the use of keystones with predefined weight ranges has been adopted for facilitating visual comparison and dispute resolution through weighed gradation. Article 1005.01 (c) suggests a maximum of $15 \%$ of the total test sample by weight may be oversized material, and each oversized piece shall not exceed the maximum permissible particle size by more than $20 \%$. In this regard, reliable field-imaging techniques are needed to easily and quickly process stockpile images of riprap for gradation checks and provide actionable data analytics.

Over the past two decades, imaging-based analysis techniques have been widely developed and adopted to characterize particle size and shape properties of aggregates from their scanned photos. These techniques have been significantly enhanced to provide high accuracy and efficiency in the characterization (Rao et al. 2002; Al-Rousan et al. 2005; Pan et al. 2006; Wang et al. 2013; Moaveni et al. 2013; Hryciw et al. 2014). Current laboratory image-based analysis approaches mostly focus on the shape analysis using camera(s) attached to table-sized equipment on which a set of prepared particles are placed for photo capture. For example, the Aggregate Imaging System (AIMS) uses a setup consisting of one slide-mounted camera and two lighting sources. The Enhanced-University of Illinois Aggregate Image Analyzer (E-UIAIA) takes three 2D images of particles on a conveyor system from three orthogonal axes where cameras are installed. Among the most widely used techniques in aggregate shape characterization are the Flat and Elongated Ratio (FER), Angularity Index (AI), and Surface Texture Index (STI). They were developed as key indices from national studies, NCHRP 4-30 and 4-34 and the TPF-5(023) pool fund, using E-UIAIA (Tutumluer et al. 2000; Rao et al. 2002; Pan et al. 2006; Moaveni et al. 2013).

Laboratory imaging systems generally use a limited number of 2D images, typically up to three, because of the fixed camera positions. As a result, the generated 3D particles may lack shape details such as local concave curvatures on the surface. Furthermore, the setups are usually not portable and are unwieldy for field inspection. While laboratory imaging equipment has been mostly used for shape characterization, in situ imaging alternatives, e.g., WipFrag (Maerz et al. 1996), have been developed to obtain the grain size distribution data in the field. For example, the WipFrag system was tried by IDOT in the field for riprap imaging with limited success because image quality and accuracy

were affected by shadows and overlaps between particles in the image. Therefore, the existing 
laboratory or in situ methods do not provide a complete set of analysis data for both particle size and shape characterization.

Because the size and shape properties of riprap and large-sized aggregates cannot be obtained with standard sieve analysis or laboratory-based image analyzer setups, a newly developed field-imaging technique was first implemented and used in recent IDOT-ICT study R27-124 (Kazmee et al. 2016). For this effort, representative particles of each aggregate subgrade material (RR01, CS01, and CSO2 per IDOT specs) with particles greater than $3 \mathrm{in} .(7.6 \mathrm{~cm})$ in size were spread out on a blue tarp in the field. High-resolution (e.g., $1292 \times 964$ pixels) images of these large aggregate particles were captured with a Digital Single-Lens Reflex (DSLR) camera. A white-colored calibration ball placed beside the aggregate particles was introduced to scale the spatial resolution properly. The final outcome of the segmentation is a simplified black and white binary image of each particle where the black pixels are the background and the white pixels are the object. Further details on this image-processing technique can be found elsewhere (Moaveni et al. 2013). The morphological indices FER, Al, and STI were computed from the extracted binary images. The dimensions of the particles were determined in reference to the spatial resolution of the calibration ball.

In view of recent advances in the area of computer vision and machine learning, machine visionbased inspection systems trained using sample images have the potential for directly identifying individual particles for shape and size analysis from stockpile or constructed layer trench views showing aggregate assemblies. For example, image segmentation has been implemented at UIUC to study individual railroad ballast particles collected in the field in terms of their shape and size variability versus depth of sampling (Moaveni et al. 2013). Through preliminary image analysis and algorithm development, individual particles can be segmented from images with overlapping particles and their size and shape properties can be quantified from the cut sections imaged from field ballast trenches. Nevertheless, the image analysis algorithm entails heavy interaction with the user for fine-tuning segmentation parameters, and the quality of the segmentation results needs to be further enhanced.

The conceptual scheme of a field-imaging-based particle size and shape analysis framework for riprap or large-sized aggregates was proposed in this project. The motivation is to devise a field evaluation procedure for capturing riprap stockpile photos and a software program to facilitate expedited processing of the particle photos. Using this evaluation tool, a single inspector can collect multiple camera views and conduct analyses for determining individual aggregate particle size and shape properties from the stockpile. The evaluation tool has been envisioned to run the proposed userindependent image analysis software on a laptop computer for obtaining robust and repeatable results.

The main benefit of this implementation project includes the ability to conduct riprap and large-sized aggregate size and shape property testing in the field, at a quarry or construction site, with improved accuracy in determining the size/shape properties from imaging without having to weigh rock pieces. This is a pressing need because IDOT engineers and field inspectors spend considerable time and effort in riprap sizing at approximately 50 sites in Illinois annually. 
Implementation of advanced field-imaging technology intended to be fully developed in this project will help to accurately determine if riprap material meets gradation specifications. The proposed system will be sustainable for field application by utilizing readily available imaging-based size and shape indices and the stockpile image-segmentation techniques to be developed. Better property characterization and optimized material selection will be achieved to improve designs through effective quality control, reduced costs, increased life cycle and sustainability with less environmental impacts, and minimum labor and energy consumption. Major cost savings in terms of personnel time, transportation, and laboratory equipment and facility use will be realized because no samples will be collected from the field for detailed laboratory analysis.

\subsection{RESEARCH OBJECTIVE}

The primary objective of this research project is to develop a convenient, nonintrusive fieldevaluation system based on imaging. An engineer or inspector can then take multiple photos of the riprap and large-sized aggregates and determine the gradation (size distribution) and shape properties with the help of calibration balls used in image analyses. The following specific goals were determined for the study:

- Identify aggregate producers of riprap and large-sized aggregates in Illinois, and acquire image data and representative samples from field visits. Gather information on the types, geological origins, and sources of the materials. Study applicable current manual methods for QA/QC.

- Develop field-imaging algorithms that accomplish user-independent segmentation for robust operation under a wide variety of riprap stockpile field conditions, e.g., strong sunlight and/or shadow considerations.

- Develop software algorithms for size and shape analyses of individual aggregate particles captured from multiple views, and integrate the algorithms into a software application with Graphical User Interface (GUI).

The final field-imaging technique was intended to be portable, affordable, and accurate for reliable data compilation under different field conditions.

\subsection{RESEARCH METHODOLOGY}

To fulfill the above-stated research objective, the following tasks and methodologies are considered in this study:

- Gathering information on the types, geologic origins, and representative sources of riprap and large-sized aggregates materials, as well as information on the statewide locations of the approved lists of these materials in Illinois. The research team worked closely with IDOT's Central Bureau of Materials (CBM) and district engineers on several quarry field visits to identify the approved aggregate sources (from RR3 to RR7 size categories as per IDOT specifications) and to collect representative samples.

- Establishing a database of riprap and large-sized aggregate images in accordance with the laboratory and field samples identified/acquired. The image database includes different 
settings of large-sized aggregates common in quarry scenes, i.e., single particle, adjacent/overlapping particles, and particle stockpiles. Representative samples are tested in the laboratory, with their volumes and weights reported as the ground-truth validation.

- Developing automated, user-independent image segmentation and morphological analysis algorithms for robust operation under a wide variety of riprap or large-sized aggregate field conditions, e.g., strong sunlight and/or shadow considerations. To achieve reliable and accurate segmentation of aggregate particles, a comprehensive literature review and trials are conducted to evaluate and determine the use of traditional computer vision techniques as well as emerging deep-learning artificial intelligence approaches.

- Validating the accuracy and robustness of the developed algorithms by comparing with measured ground-truth data. Comparisons are made for good performance and reliable accuracy of the algorithms from engineering practice.

- Designing and developing a software application as the integrated riprap aggregate evalution module for size/shape analyses of individual particles captured in stockpile images. A graphical user interface (GUI) is designed for the ease of user interaction.

\subsection{REPORT ORGANIZATION}

This report consists of seven chapters, including this introductory chapter.

Chapter 2, titled "Literature Review," provides a review of riprap standards and specifications, past aggregate studies and equipment that leverage imaging techniques, and the applications of artificial intelligence and deep-learning techniques.

Chapter 3, titled "Field Studies and Sampling of Riprap Material in Quarries," provides an overview of field studies and the laboratory component of this project. This chapter includes riprap source information from the riprap production sites visited by the research team, material selection and image-acquisition criteria, and laboratory tests for measuring the ground-truth data of collected samples.

Chapter 4, titled "Single-Particle Volumetric Estimation Using Computer Vision," provides the algorithmic details of the single-particle segmentation, volumetric reconstruction approach, and the related validation results. This chapter introduces the development of a computer vision-based approach for the volumetric measurement of individual riprap particles, which uses a riprap database as ground-truth data for development as well as future research.

Chapter 5, titled "Riprap Stockpile Particle Size and Shape Estimation Using Deep Learning," provides the algorithmic details of the stockpile segmentation and morphological analyses approach and the verification results with ground-truth manual labeling. This chapter also includes the established stockpile labeled image dataset, the training of a deep-learning-based automated segmentation approach, the development of morphological analysis modules, and the completeness and accuracy analyses of the results. 
Chapter 6, titled "Software Development for Riprap Stockpile Characterization," provides a detailed description of the software development stages undertaken in this project, which includes the software engineering concepts adopted, the graphical design and control logic of the software application, and the back-end implementation details of the cross-platform software.

Chapter 7, titled "Conclusions and Recommendations," provides a summary of research findings and the main recommendations and conclusions from this study. This chapter also discusses the next steps to further extend this study and recommendations for future research. 


\section{CHAPTER 2: LITERATURE REVIEW}

\subsection{INTRODUCTION}

This chapter presents a review of the riprap quality assurance/quality control (QA/QC) requirements and previous research studies related to the imaging techniques and developed systems used in aggregate research and emerging artificial intelligence-based approaches. An overview of the current practices for characterizing riprap and large-sized aggregate materials is also discussed.

\subsection{APPLICATIONS AND SPECIFICATIONS OF RIPRAP AND LARGE-SIZED AGGREGATES}

Riprap rock and large-sized aggregates, quarried from undisturbed, consolidated rock deposits or recycled from demolished concrete construction, have been used extensively in geotechnical and hydraulic engineering to armor streambank revetment, bridge piers, and abutments, as well as other coastal structures. They serve as an important functional component by providing water/ice erosion control, sediment control, rockfill, and scour protection against hydraulic and environmental stresses (IDOT 2016). As a natural material, the reliable and sustainable use of riprap as an integrated system requires quality control throughout the design, production, transport, installation, inspection, and maintenance stages (Lagasse et al. 2006). Apart from the structural geometry, slope stability, and hydraulic analyses of the structures, case studies on riprap failure in stream channels and bridge piers indicate that undersized particles and open-graded riprap often cause insufficient resistance to hydraulic shear stress (Blodgett and McConaughy 1986; Chiew 1995; Richardson and Davis 2001; Lagasse et al. 2001).

During the material selection and QA/QC process, characterizing particle size and shape properties has become a focal point for aggregate studies. Particle size and morphological/shape properties of aggregates primarily influence the macroscopic behavior and performance of aggregate skeleton assemblies of constructed layers in transportation infrastructure, e.g., asphalt concrete and Portland cement concrete (Quiroga and Fowler 2004; Polat et al. 2013), unbound/bound layers in highway and airfield pavements (Liu et al. 2019; Tutumluer and Pan 2008; Bessa et al. 2014), the ballast layer in railway tracks (Huang 2010; Wnek et al. 2013), and riprap materials for erosion control and hydraulic applications (Lutton et al. 1981; Lagasse et al. 2006). Across all size ranges, aggregate shape properties in terms of form (e.g., flatness and elongation), angularity, and texture have been used to characterize their morphology (Barrett 1980). Information on aggregate morphology greatly facilitates the quality control process and in-depth understanding of aggregate layer behavior linked to particle composition and packing.

For producers and practitioners, the size and shape of aggregate sources are important for QA/QC requirements throughout the production line and mix design (ASTM D448 2017; ASTM D2940 2015; ASTM D6092 2014). Different quarrying processes and rock mineralogy introduce randomness to the quality of produced aggregates. Therefore, convenient and continuous monitoring of quarry products is important for efficient material selection and construction. On the other hand, particulate mechanics dealing with discrete aggregate particle interactions and realistic modeling of assembly behavior of granular materials require properly characterizing the morphological properties of 
aggregates. Through recently focused research efforts on modeling the aggregate layer behavior using the Finite Element Method (FEM) and Discrete Element Method (DEM), aggregate morphological properties have gained increased importance, after grain size distribution, for establishing an accurate composition and packing that enable the modeling scheme to capture complex behaviors of granular materials. This is especially challenging for most stone skeleton layers in constructed road pavements, e.g., surface course mixtures such as hot-mix asphalt (HMA) and Portland cement concrete (PCC) and unbound aggregate base/subbase, which are subjected to vehicular dynamic loading conditions (Huang 2010; Chen 2011; Ghauch 2014; Qian 2015). Therefore, uniform guidelines, specifications, or techniques that ensure reliable and efficient characterization of weight, size, shape, and gradation of different aggregate categories are critical in quarry production lines and construction sites (Lagasse et al. 2006).

Despite the ongoing development of guidelines for size selection of riprap in design, the practical procedures for characterizing riprap size and shape properties in the field are still subjective and qualitative, primarily because of difficulties associated with measuring sizes of these large particles/rocks. Determination of particle-size distribution or gradation of riprap and other large-sized aggregate is continually difficult in the field. The riprap gradation or sizing of the pieces shall be wellgraded, as outlined in Article 1005.01(c) of IDOT's Standard Specifications for Road and Bridge Construction (IDOT 2016). Individual rock pieces are measured for weight, which is a time-consuming and labor-intensive task. Sample grid systems have been used for gradations of RR3 to RR7 with less success. As compared to coarse aggregates used in transportation engineering, the sizes of which

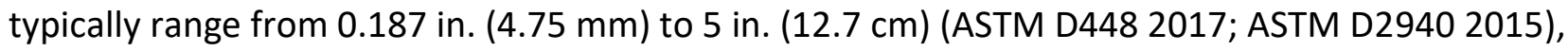
individual riprap rock can weigh up to $1,150 \mathrm{lbs}$. $(522 \mathrm{~kg}$ ) with nominal sizes up to $24 \mathrm{in}$. $(61.0 \mathrm{~cm}$ ) (IDOT 2016; ASTM D6092 2014). Laboratory sieve analysis is usually conducted to determine the gradation of small- to medium-sized aggregates, but large-sized riprap material makes this task impractical. Because there is no uniform way to define the size dimension of individual rocks, standards or guidelines usually specify riprap gradation requirements in terms of weight.

At the current state of the practice, a nationwide AASHTO survey of transportation agencies in the United States and Canada has indicated that riprap characterization is mostly based on visual inspection and hand measurements (Sillick 2018). Visual inspection depends greatly on the experience and expertise of practitioners. In this method, certain gauges or keystones and sample stockpiles are usually used as a reference to assist the judgement (Lippert 2012). To better estimate size distribution, the Wolman count method is applied by statistically sampling and measuring rocks within a stockpile (Lagasse et al. 2006). For hand measurement, transportation agencies either weigh individual particles directly or use size-mass conversion after measuring particle dimensions. The piece size-mass conversion proposed in ASTM D5519 (2015) requires measurement of the midway dimension or circumference from three orthogonal axes and estimates the volume based on a cuboid assumption or averaged sphere-cube assumption. However, visual inspection and hand measurement provide rough estimations that do not necessarily represent realistic riprap properties, and an objective and efficient approach for quantitatively characterizing the size and shape of riprap has yet to be established. 


\subsection{ADVANCED AGGREGATE IMAGING SYSTEMS}

Over the past two decades, imaging-based analysis has been widely developed and adopted to characterize particle size and shape properties from the digital images of aggregates. Related imagesegmentation techniques have been significantly enhanced to provide high accuracy and efficiency in practice (Rao et al. 2002; Al-Rousan et al. 2005; Pan et al. 2006; Wang et al. 2013; Hryciw et al. 2014). Most imaging techniques for image analysis are developed and applied to aggregates with maximum particle sizes less than 6 in. $(15.2 \mathrm{~cm})$ using a fixed-position camera setup for image acquisition in the laboratory. Masad (2003) and Gates et al. (2011) developed the Aggregate Imaging System (AIMS), which consists of one slide-mounted camera and two lighting sources visualizing a maximum particle size up to 6 in. $(15.2 \mathrm{~cm})$. Tutumluer et al. (2000) originally developed and later Moaveni et al. (2013) improved the Enhanced-University of Illinois Aggregate Image Analyzer (E-UIAIA). This system takes three $2 \mathrm{D}$ images of particles on a conveyor system, where cameras are installed along three orthogonal axes, and processes the set of orthogonal views for each particle, with a maximum particle size up to 3 in. $(7.6 \mathrm{~cm})$. Komba et al. (2013) established a 3D laser-based aggregate system that scans an individual particle and analyzes the generated 3D mesh model, with a maximum detected sample size of $0.75 \mathrm{in}$. $(1.9 \mathrm{~cm})$. Hryciw et al. (2014) used a Translucent Segregation Table (TST) and evaluated the effect of particle size and morphology on the shear strength mechanical properties of the material. Zheng and Hryciw (2014) introduced an algorithm to accurately determine the thickness as well as the size characterization of particles ranging from sand to gravel (maximum size of $1.2 \mathrm{in.}[3.0 \mathrm{~cm}])$ simultaneously. Obaidat et al. (2017) established a smartphone-based scheme that utilizes image-processing techniques and ImageJ commercial software to extract coarse aggregate properties, with a maximum particle size up to $2 \mathrm{in}$. $(5.1 \mathrm{~cm})$. Jin et al. (2018) developed an algorithm to perform aggregate shape characterization and volume estimation based on a 3D solid model constructed from X-ray CT images.

Although these imaging systems were developed and validated with ground-truth measurements under laboratory conditions, their capabilities for field application has not been verified. First, these systems are designed with a laboratory-scale setup for object and camera positioning. Thus, they may not be easily transported, assembled, and deployed for field applications, especially for those involving advanced devices such as a 3D-laser scanner or an X-ray CT scanner. Moreover, most of the systems have a maximum particle-size restriction, limiting their application for handling large-sized aggregates. Further, the lighting conditions for these systems are controlled using backlighting or multiple light sources to minimize the shadow and reflection effects. In addition, the imagesegmentation algorithm originally developed for laboratory conditions may also have difficulties with accuracy and robustness under field lighting conditions. Consequently, laboratory imaging systems are not directly applicable or adaptable for field inspection.

Furthermore, most laboratory imaging systems focus on 2D particle size and shape analysis instead of 3D volumetric information, although the weight of individual rocks is needed for determining size distribution of riprap material. Note that the WipFrag software developed by Maerz et al. (1996, 1999) and commercialized by WipWare Inc. is the only imaging-based system found in the literature that was used to provide riprap characterization based on field images. It was integrated with mobile devices to allow for convenient on-site use to roughly estimate the particle sizes and gradation in a stockpile image. Nevertheless, the image-segmentation procedure used in this software is highly user 
dependent and its gradation property estimation is based on a single-view stockpile image of riprap, without accounting for detailed 3D particle shape or volumetric information.

Among these emerging image-based techniques, a field-imaging system with robust and efficient algorithms for obtaining comprehensive 3D volumetric information of aggregate particles-especially riprap and large-sized aggregates - has not yet been developed.

\subsection{IMAGE-PROCESSING APPROACHES FOR AGGREGATE EVALUATION}

Traditional methods for aggregate evaluation include visual inspection, geometry measurements, and sieve analysis, while the accurate characterization of particle shape is challenging and labor-intensive for humans to visually or manually determine. In this regard, computer vision techniques have been widely adopted to characterize particle size and shape properties, and a variety of aggregate imaging systems have been developed.

Aggregate image analysis typically consists of an image-segmentation module followed by a morphological analysis module based on computational geometry (Al-Rousan et al. 2007). Image segmentation extracts the interested region of target particle(s) from the image background, which is a key step for extracting information from the image-acquisition step. The setups of these systems are usually configured to ensure spacing among particles such that the effort required to separate overlapping or touching particles during the image segmentation is minimized. The AIMS system (Masad et al. 2007; Gates et al. 2011) can capture multiple aggregates manually spread onto a tray. Coarse aggregates are spread as separated particles, while fine aggregate particles may touch each other. Further post-processing is required by conducting the convex hull test. The E-UIAIA system (Tutumluer et al. 2000; Moaveni et al. 2013) acquires aggregate photos from orthogonal views of individual particles only. Huang et al. (2019) recently developed a field-imaging system for large-sized aggregates following a similar design concept. These variations of UIAIA deal with single-particle imaging with no touching or overlapping involved. Other imaging systems such as a 3D laser-based system by Anochie-Boateng et al. (2013) and a stereo photography-based system by Zheng and Hryciw (2017) mainly focused on aggregate particles with minimal contact or overlap.

The above aggregate imaging systems manually control the arrangement of particles and achieve high-precision measurements of separated or non-overlapping aggregates. However, when aggregates are in a densely stacked or stockpile form, which are the more practical scenarios, their capability to simultaneously characterize a large quantity of aggregates may not be sufficient for several reasons. First, these systems manually separate the particles and provide a constant background to simplify the image-segmentation task. This condition can no longer be satisfied when aggregates are in a stockpile background or other field scenes. Second, manually feeding many particles into these systems is inefficient for time-sensitive monitoring applications. Moreover, the application of these advanced imaging systems is further limited when only in-place evaluation is available at production or construction sites or when characterization of large-sized aggregates is needed.

To overcome the challenges of analyzing stockpile aggregate images, more advanced imagesegmentation techniques are required. Traditional 2D image-segmentation methods include three 
major types, region-based, edge detection-based, and watershed, among which the variations of edge-based and watershed segmentation algorithms have been shown to perform better in the presence of mutually touching particles in dense images such as stockpile aggregate views (Wani and Batchelor 1994; Senthilkumaran and Rajesh 2009; Vincent and Soille 1991). In this connection, several research software systems and industrial applications have been developed. For example, Tutumluer et al. (2017) and Huang et al. (2018) applied watershed segmentation to characterize the degradation level in trench-view images of railway ballast by classifying the size distributions of image segments. Similarly, the commercial cross-platform software WipFrag, developed by Maerz et al. (1996, 1999), uses edge-based segmentation to partition rock fragments and estimate particle-size distribution in a stockpile image. Nevertheless, both image-segmentation algorithms used in the software programs are user-dependent. Considerable user interaction by either parameter fine-tuning or interactive editing is required to achieve an acceptable segmented image.

\subsection{MACHINE-LEARNING FACILITATED COMPUTER-VISION TECHNIQUES}

Over the last decade machine learning-based methods have enabled significant advances in many complex vision tasks benefitting from the development of artificial intelligence and computer vision techniques (Prince 2012). Dense image-segmentation tasks, along with many object classification and detection tasks, are difficult in the sense that the features in the image are usually implicit and thus cannot be easily extracted and represented by human means. While traditional segmentation methods are not effectively applicable to identify these features, machine learning methods may better handle such tasks by capturing the underlying features based on data-driven mechanisms. Zheng and Hryciw (2018) proposed an approach using pattern recognition to identify particles in images of sand assemblies. The approach followed the Voila-Jones object detection framework, which is a combination of traditional feature extraction techniques and machine learning algorithms. However, this approach still requires careful design of a feature extractor that transforms the raw image into effective internal representation.

During recent developments in the computer vision domain, a deep-learning framework proposed by LeCun et al. (2015) exhibits several advantages over conventional machine learning techniques because of its better capability of discovering intricate structure in large datasets with minimal human-guided interaction. With multiple levels of abstraction in the neural network, deep learning has dramatically improved the state of the art in many complicated tasks in computer vision, such as image classification, object detection, semantic segmentation, etc. Considering this fact, applying deep-learning techniques to stockpile aggregate image-segmentation tasks is promising and has not yet been given its due consideration.

\subsection{SUMMARY}

This chapter provided a review of riprap standards and specifications, findings from previous aggregate studies, relevant equipment that leveraged imaging techniques, and the applications of machine learning-based technology. 
Traditional methods for assessing riprap geometric properties involve subjective visual inspection and time-consuming hand measurements. As such, achieving comprehensive in situ characterization of riprap materials remains challenging for practitioners and engineers. In this regard, several advanced aggregate imaging systems developed over the years utilized computer vision techniques to approach this task in a quantitative, objective, and efficient manner.

In terms of aggregate imaging, particle size and morphological/shape properties ensure the reliable and sustainable use of all aggregate skeleton materials placed as constructed layers in transportation applications. Composition and packing of these aggregate assemblies rely heavily on particle size and morphological properties, which impact layer strength, modulus and deformation response under vehicular loading, and therefore facilitate the QA/QC process. Aggregate imaging systems developed to date for size and shape characterization, however, have primarily focused on measurement of separated or non-overlapping aggregate particles. The development of efficient computer vision algorithms based on traditional computer vision techniques and/or emerging deep-learning techniques is urgently needed for image-based evaluations of densely stacked (or stockpile) aggregates, which requires image segmentation of a stockpile for the size and morphological properties of individual particles. 


\section{CHAPTER 3: FIELD STUDIES AND SAMPLING OF RIPRAP MATERIAL IN QUARRIES}

\subsection{INTRODUCTION}

This chapter presents details for the acquisition of samples used for studying the shape and size properties of IDOT riprap materials. An overview of IDOT's riprap gradation requirements and size/weight categories are first introduced. Information on the field visits to aggregate producer sites to characterize, image, and collect riprap particles of different particle sizes and shape properties are given next. Lastly, the details for riprap imaging and data acquisition were discussed for single-rock and stockpile image acquisition. An explanation of imaging setups, data acquisition procedures, and required measurements are discussed in this chapter.

\subsection{OVERVIEW OF IDOT RIPRAP SIZE AND SHAPE REQUIREMENTS}

The current IDOT specification for riprap classification into different "RR" categories is based on the grain size distribution, which is determined by the weight distribution of the riprap stones. IDOT published a policy memorandum in 2012 for the classification of riprap based on weight (Policy Memorandum No. 14-08.1). The latest revision of this memorandum on July 24, 2018, requires a visual inspection of the riprap stockpiles, including inspections for flat and elongated pieces (Policy Memorandum No. 14-08.2). A collection of riprap keystones shall be maintained by the producers for all produced riprap gradations, as outlined in Table 3.1, to assist with the visual gradation. IDOT requires that the set of keystones shall be representative of the stockpile gradation and be replaced with a new set if they become nonrepresentative.

Table 3.1. Keystone Requirements for Different Riprap Size/Weight Categories

\begin{tabular}{cccc}
\hline Gradation & Keystone \#1 (lbs.) & Keystone \#2 (lbs.) & Keystone \#3 (Ibs.) \\
\hline RR3 & $50( \pm 5)$ & $10( \pm 1)$ & $1( \pm 0.1)$ \\
RR4 & $150( \pm 15)$ & $40( \pm 4)$ & $1( \pm 0.1)$ \\
RR5 & $400( \pm 40)$ & $90( \pm 13)$ & $3( \pm 0.1)$ \\
RR6 & $600( \pm 60)$ & $170( \pm 17)$ & $6( \pm 0.5)$ \\
RR7 & $1000( \pm 100)$ & $300( \pm 30)$ & $12( \pm 1)$ \\
\hline
\end{tabular}

Note: $1 \mathrm{lb} .=453.6$ grams

If the gradations by visual inspection were disputed by the producer, then a second visual inspection is conducted by Central Bureau of Materials (CBM). If the second visual inspection is again disputed by the producer, then a representative sample is excavated from the working face of the stockpile and spread over the length of a marked grid to a one-rock thickness and weighed piece by piece for riprap categories RR3-RR7. The rock spalls and fines below the minimum specified weight are collected and included in the calculations for each size range. The grid size for each riprap category is outlined in Table 3.2. The grid length is broken into 5 in.-long blocks ( $127 \mathrm{~mm}$ ). Note that for riprap 
categories RR1 and RR2, the grain size distribution is performed by conventional sieve analysis in accordance with Illinois Test Procedure 27, outlined in IDOT's Manual of Test Procedures for Materials (2017).

Table 3.2. Grid Size Requirements for Sampling Different Riprap Gradation Categories

\begin{tabular}{ccc}
\hline Gradation & Grid Size (ft) & (Min. Number of Tested Blocks) \\
\hline RR3 & $2^{\prime}$ by $25^{\prime}$ & 2 \\
RR4 & $3^{\prime}$ by $25^{\prime}$ & 2 \\
RR5 & $4^{\prime}$ by $25^{\prime}$ & 3 \\
RR6 & $5^{\prime}$ by $30^{\prime}$ & 3 \\
RR7 & $5^{\prime}$ by $35^{\prime}$ & 3 \\
\hline
\end{tabular}

Note: $1 \mathrm{ft}=30.48 \mathrm{~cm}$

Based on IDOT policy memorandum, the procedure for riprap size characterization entails using three keystone particles (used as control points) to identify gradations. Figure 3.1 shows the upper, lower, and midpoint gradation lines for IDOT's riprap categories RR3-RR7. This plot assumes a flat and elongated ratio of $2: 1$, a specific gravity of 2.5 , and an ellipsoidal particle shape for a standardized weight to volume/size conversion. Note that the maximum dimension of a particle is used to indicate size on the horizontal axis.

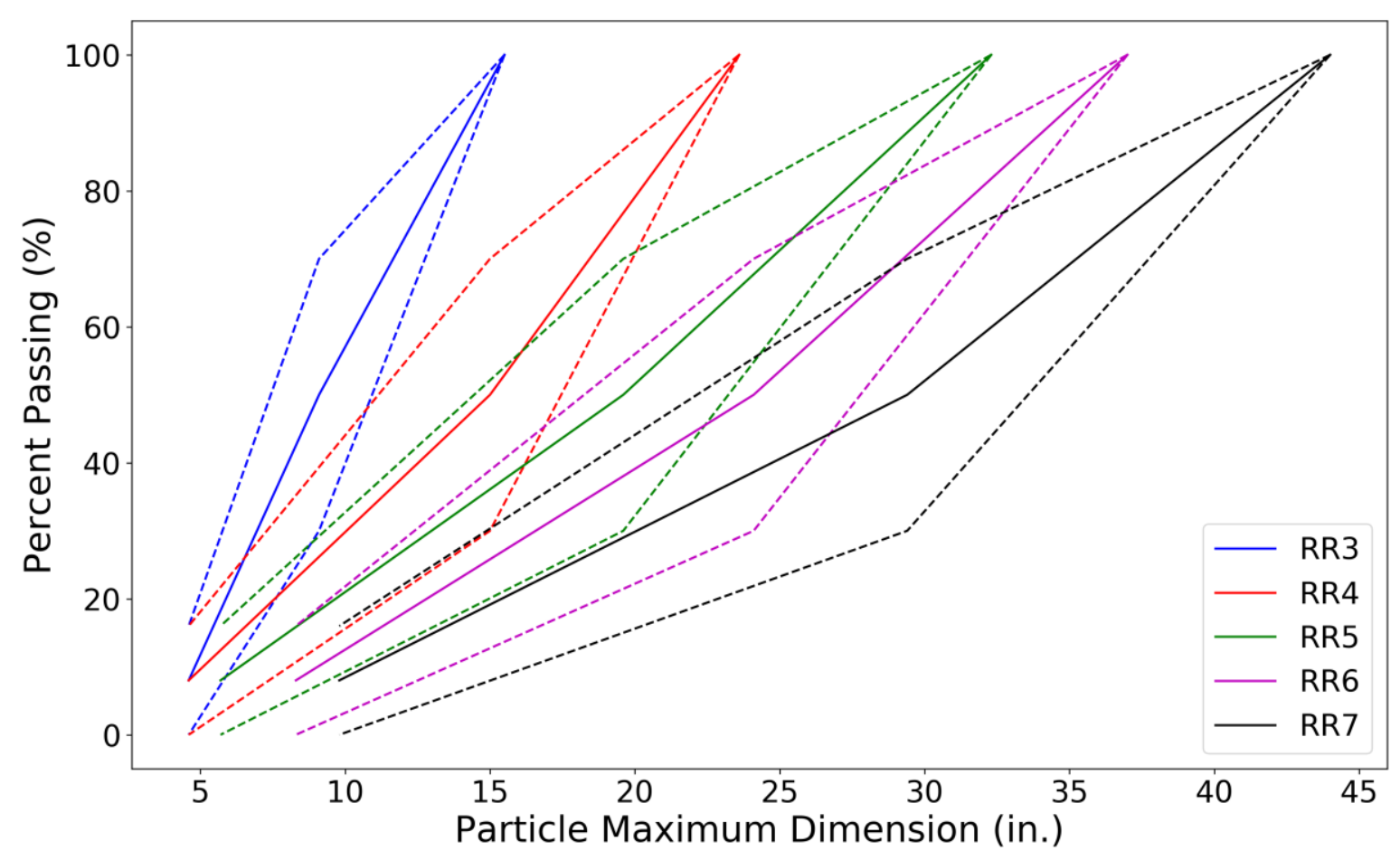

Figure 3.1. Converted particle-size distribution of IDOT riprap categories RR3-RR7. 


\subsection{SELECTION OF RIPRAP MATERIAL AND AGGREGATE PRODUCERS}

Several field visits were made to aggregate quarries in Illinois to sample and image riprap rocks and stockpiles in order to establish a robust and reliable database for determining their realistic size and shape properties. In addition, existing image libraries of riprap stockpiles from quarries in Illinois were collected from aggregate producers. These image libraries were mostly established by quarries from previous QA/QC activities.

To develop robust imaging algorithms progressively, the study was carried out in two stages. At first, shape and size properties were collected and validated for individual large-sized rocks. Once a good correlation was established between the imaged particles and the ground-truth measurements, the second stage was to image and segment stockpiles of riprap rocks, with touching and overlapping particles. During the field visits, riprap images specifically purposed for each stage should be collected, requiring a careful selection of riprap aggregate producers for the visits. Information gathered on the selected IDOT-approved riprap aggregate producers, locations, gradations, and quality (A or B) were collected and plotted in Figure 3.2. This map provides geographic information for site selection and data acquisition of riprap sources and images.

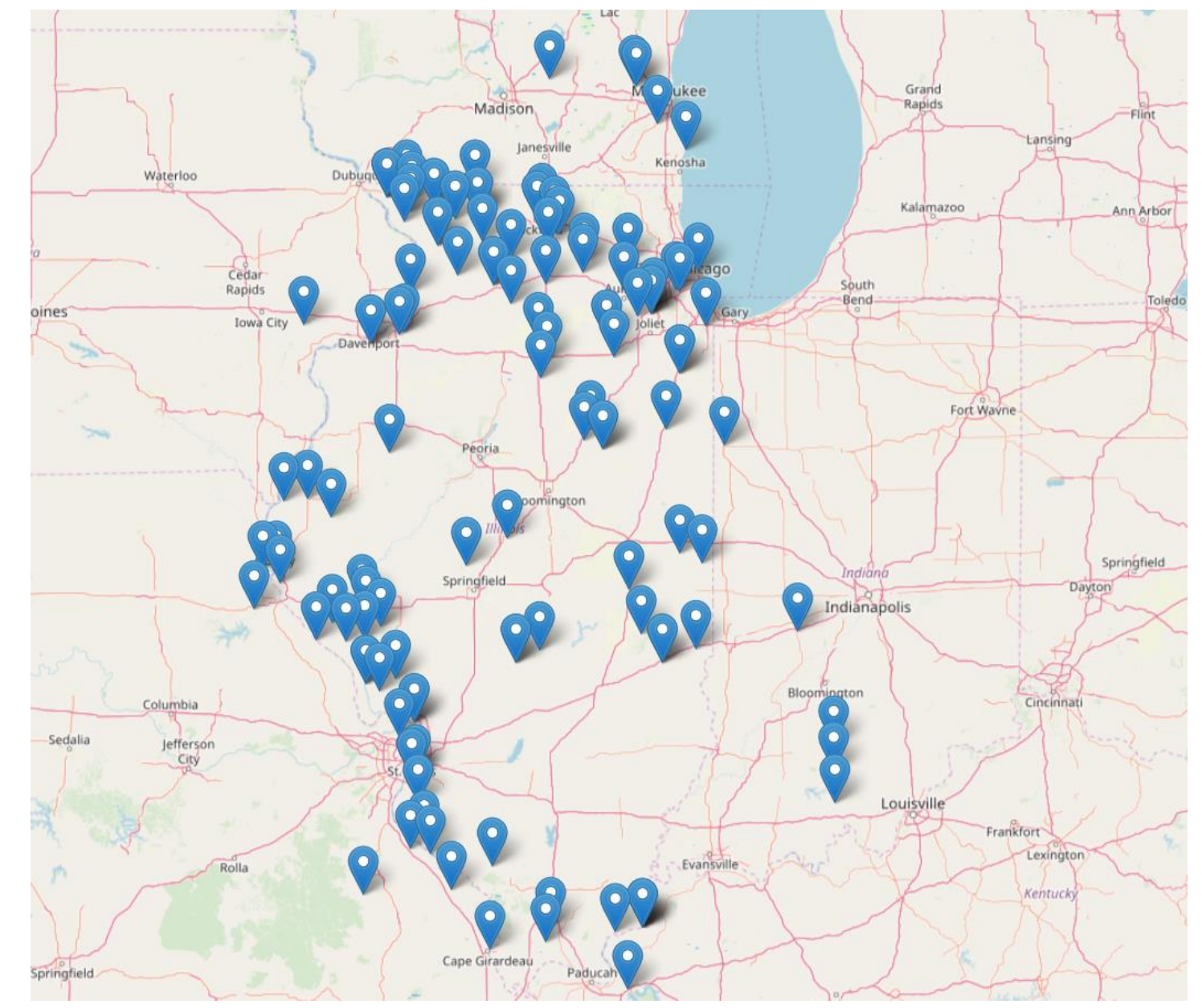

Figure 3.2. Geographical information for selected IDOT-approved aggregate producers (IDOT's Approved/Qualified Producer List of Aggregate Sources 2018). 


\subsubsection{Riprap Sources for the Single-Rock Study}

For the first stage, i.e., the single-rock study, images of individual large rocks were taken. In total, 85 particles were selected and analyzed from two sources. All particles were analyzed using an inspection system (detailed later in this chapter), and ground-truth volume/weight information was measured. The purpose of this study was to evaluate the feasibility of the inspection system and validate the robustness of the associated segmentation and reconstruction algorithms.

The first source was a stockpile of aggregate subgrade material conforming to IDOT CSO2 gradations obtained from Vulcan materials in Lisbon, Illinois. The stockpile was previously used in the recent project ICT-R27-168 for rockfill applications. More details about the source properties are given elsewhere (Qamhia et al. 2018). Only particles larger than 3 in. (76.2 mm) were selected for this study. Thus, the collected 40 rocks complied to IDOT's RR3 riprap size category. The second source was RiverStone Group, an aggregate producer in Moline, Illinois. A field trip was arranged to this quarry site to sample and image the individual rocks and collect images of riprap stockpiles. For this source, 40 rocks complying to IDOT's RR3 size category and five rocks complying to the RR5 category were selected for imaging and manual size/weight measurements. The material size and source information for the riprap rocks collected for the single-rock study is summarized in Table 3.3.

Table 3.3. Material Size and Source Information for the Single-Rock Study

\begin{tabular}{lccc}
\hline Aggregate Producer & Source Name & Number of Rocks & Size Range (in.) \\
\hline $\begin{array}{l}\text { Vulcan Materials Company, } \\
\text { Lisbon, IL }\end{array}$ & Source 1 & 40 & {$[3,6]$} \\
RiverStone Group, Milan, IL & Source 2 & 40 & {$[5,16]$} \\
RiverStone Group, Milan, IL & Source 2-Large & 5 & {$[16,26]$} \\
\hline Note: 1 in. $=2.54 \mathrm{~cm}$ & & &
\end{tabular}

\subsubsection{Riprap Sources for the Stockpile Study}

For the second stage, i.e., the riprap stockpile study, images of riprap stockpiles were taken at four Illinois quarries. Sources were selected that covered a wide variety of geological origins, riprap size categories, rock sizes, textures (shape properties), and rock colors. For each aggregate producer, several images were taken for riprap stockpiles of different sizes and from different viewing angles. The details of aggregate producers, riprap size categories, and description of riprap rocks are given in Table 3.4. The number of images reported in this table include multiple images taken at the same stockpile from different viewing angles. Note that different rock types and colors typically quarried in Illinois were chosen so that the stockpile images can be later used to train a neural network to detect different rock shapes, colors, and sizes in riprap stockpile images. Also note that two rock sizes were generally considered: medium-sized riprap rocks, ranging in weight between 1 and $40 \mathrm{lbs}$. (0.45 and $18.1 \mathrm{~kg})$, and large-sized rocks, ranging in weight between 40-600 lbs. (18.1-272.2 kg).

In addition to the stockpile images taken from the field visits, other images for riprap stockpiles conforming to different gradation categories were compiled by this project's TRP members from multiple quarries. These quarries include Prairie Materials, Ocoya; Prairie Materials, Manteno; Vulcan 
Materials, Manteno; and Vulcan Materials, Kankakee. These images were also used to train and/or validate the robustness of the associated segmentation and reconstruction algorithm.

Table 3.4. Source Information and Description of Stockpile Aggregate Image Dataset

\begin{tabular}{lcc}
\hline Aggregate Producer & Riprap Gradation and Categories & Rock Description \\
\hline Prairie Material - Ocoya, IL & RR4, RR5, and RR6 & Limestone, bluish gray, medium-sized $^{*}$ \\
$\begin{array}{l}\text { RiverStone Group, Allied Stone - } \\
\text { Milan, IL }\end{array}$ & RR4, RR5, and RR6 & Dolomite, white, medium-sized \\
$\begin{array}{l}\text { RiverStone Group, Midway Stone } \\
- \text { Hillsdale, IL }\end{array}$ & RR6 $^{+}$ & $\begin{array}{l}\text { Dolomite, yellowish to bluish gray, } \\
\text { fossiliferous, large-sized }\end{array}$ \\
$\begin{array}{l}\text { Vulcan Materials Company - } \\
\text { Kankakee, IL }\end{array}$ & RR4, RR5, RR6, and RR7 & Dolomite, yellowish, large-sized \\
\hline
\end{tabular}

* Note: "Medium sized" refers to riprap aggregates each weighing between $1 \mathrm{lb}$. (0.45 kg) and $40 \mathrm{lbs}$. (18.1 kg);

"Large sized" refers to riprap aggregates each weighing between $40 \mathrm{lbs}$. (18.1 kg) and $600 \mathrm{lbs} .(272.2 \mathrm{~kg})$;

+ Images were taken from separate, adjacent, and stockpile views.

\subsection{RIPRAP IMAGE DATA COLLECTION}

Riprap image data were collected from multiple field visits as well as from QA/QC photo libraries maintained by several aggregate producers. All active Illinois aggregate producers that participated in this study, previously listed in section 3.3, are identified in Figure 3.3. This section describes the procedure for collecting images for the single rocks using an inspection system, the laboratory testing and field size/weight measurements conducted for validating the segmentation and reconstruction algorithms, and the detailed procedure for collecting proper images for riprap stockpiles.

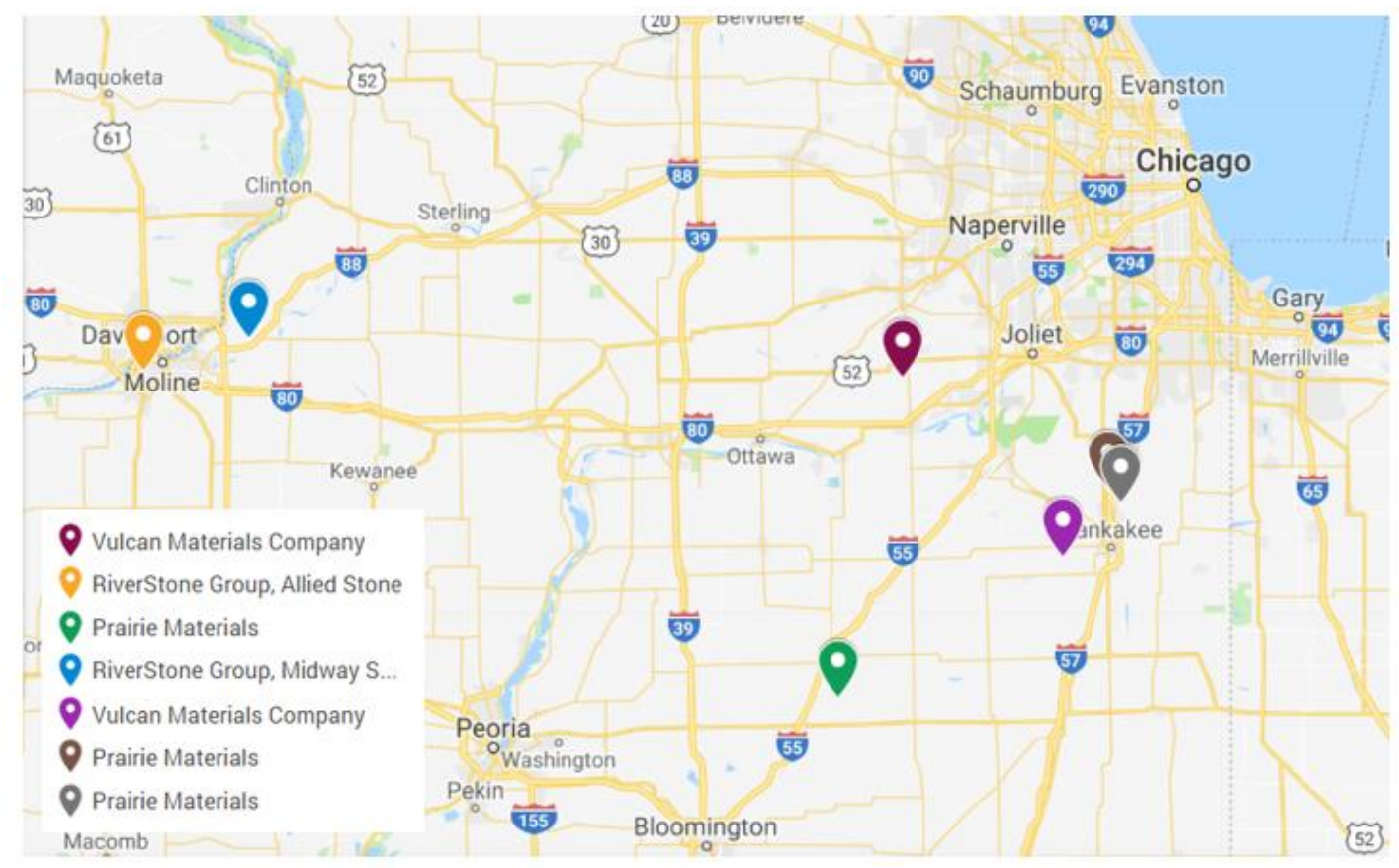

Figure 3.3. Geographical information for the aggregate producers that participated in this study. 


\subsubsection{Single-Rock Image Acquisition, Sampling, and Measurement Procedure}

An imaging-based riprap inspection system was designed and built to acquire field images of individual riprap rocks. The schematic drawing and actual photo of the field-inspection system are shown in Figure 3.4. The system consists of five major parts:

- Three smartphones with high-resolution cameras and remote shutter control.

- Three pieces of $5 \mathrm{ft}$. (1.52 m) copper tubing. The copper-tubing framework was connected by plumbing joints and can be easily assembled and disassembled for mobility.

- A $22 \mathrm{lb} .(10 \mathrm{~kg}$ ) patio umbrella base as an anchorage for the copper tubing framework.

- Three $5 \mathrm{ft}$. by $5 \mathrm{ft}$. (1.52 $\mathrm{m}$ by $1.52 \mathrm{~m}$ ) blue curtains as the background and bottom surfaces.

- Three camera tripods for fixing smartphones to the top/front/side. (One tripod was designed with a cantilever arm for holding the phone from a top view.)

The inspection system was developed to image the selected 85 riprap single rocks. The selected rock samples were intended for a comprehensive database-which covers both medium- and large-sized particles-for a reasonable validation of the algorithms. Note that only a small number of large-sized particles were inspected because of limited access to operational machinery at the quarry site for hauling rocks to the inspection system and rotating them between trials. Therefore, most riprap samples for field validation were medium-sized particles rather than large riprap rocks, such as the one shown in Figure 3.4(b).

In the beginning of the field-imaging procedure, three smartphones were aligned to achieve approximately orthogonal views. A white-colored calibration ball having a $1.5 \mathrm{in}$. $(38.1 \mathrm{~mm})$ diameter was first captured as the standard reference object. Riprap particles were then placed at the same location as the calibration ball and captured in sequence by triggering the shutter. For each riprap sample in Source 1 and Source 2 (see Table 3.3), the top-front-side image triplet was repeated three times, each time rotating the particle to a random angle. The purpose of this rotate-repeat testing is to check the reproducibility of the imaging procedure and to further investigate the variations resulting from viewing the single rocks from different angles.

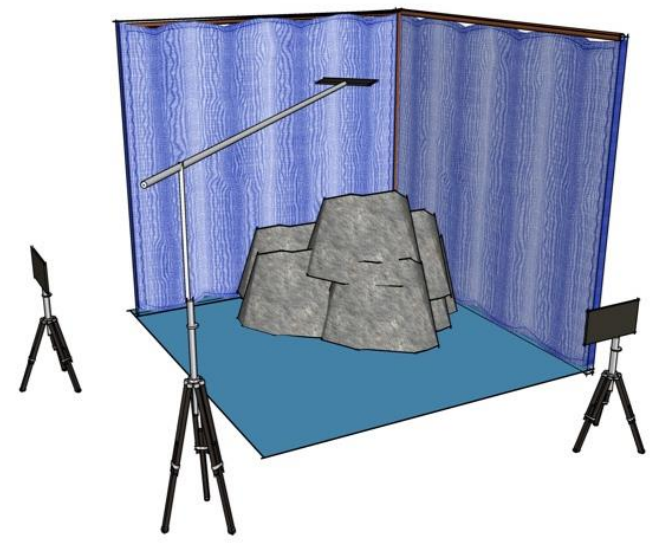

(a)

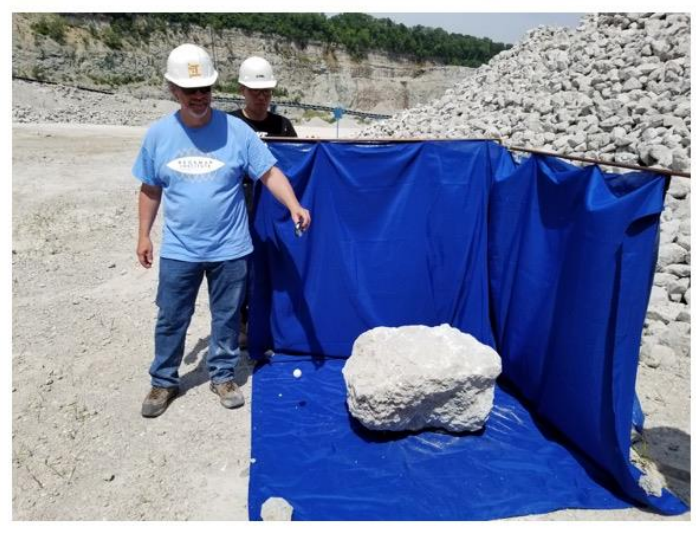

(b)

Figure 3.4. (a) A conceptual illustration and (b) the actual constructed setup of the field-imaging system for single rocks. 
The accuracy of size (and shape) measurements were checked by manual measurements of individual riprap rock dimensions and weights. As the ground-truth data, all particles in Source 1 were collected from the quarry site, and specific gravity tests as per ASTM C127 (2015) were conducted to obtain weight, volume, and specific gravity information. For Source 2 and Source 2-Large riprap rocks, only the weight of individual rocks was measured on-site. Manual measurements for rock dimensions were also conducted by experienced IDOT engineers on 20 randomly selected rocks from Source 2 and on all Source 2-Large rocks as the state-of-the-practice result. The dimension measurements were taken at three midway locations as determined by rough estimates and by the judgment of experienced IDOT engineers and practitioners. Figure 3.5 shows the current practice of measuring riprap size and weight in the field, which was used to establish the ground truth for Source 2 and Source 2-Large riprap rocks. Note that a tripod scale system shown in Figure 3.5(b) was specially designed as the best practice of IDOT for a heavy-duty weight measurement setup.

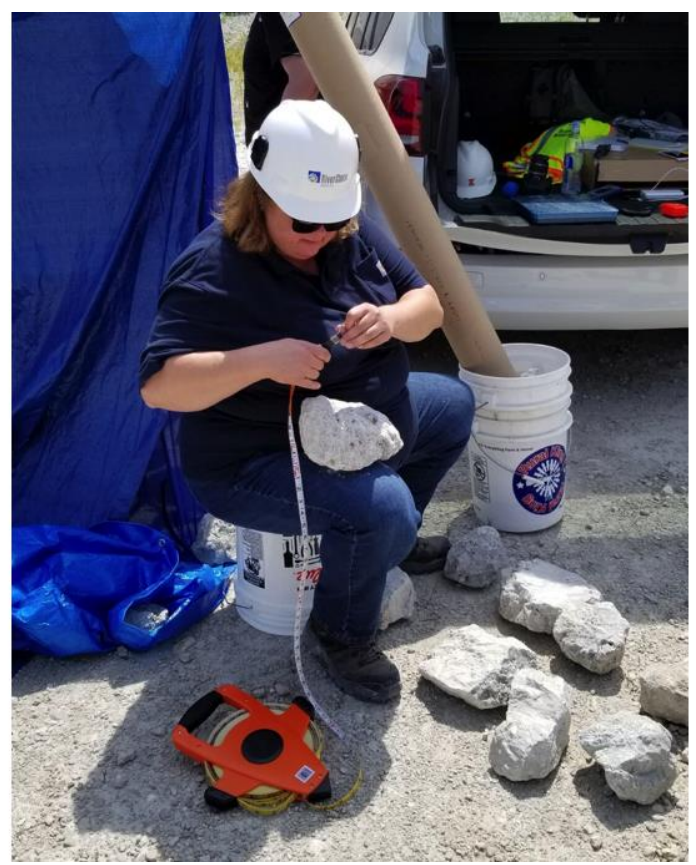

(a)

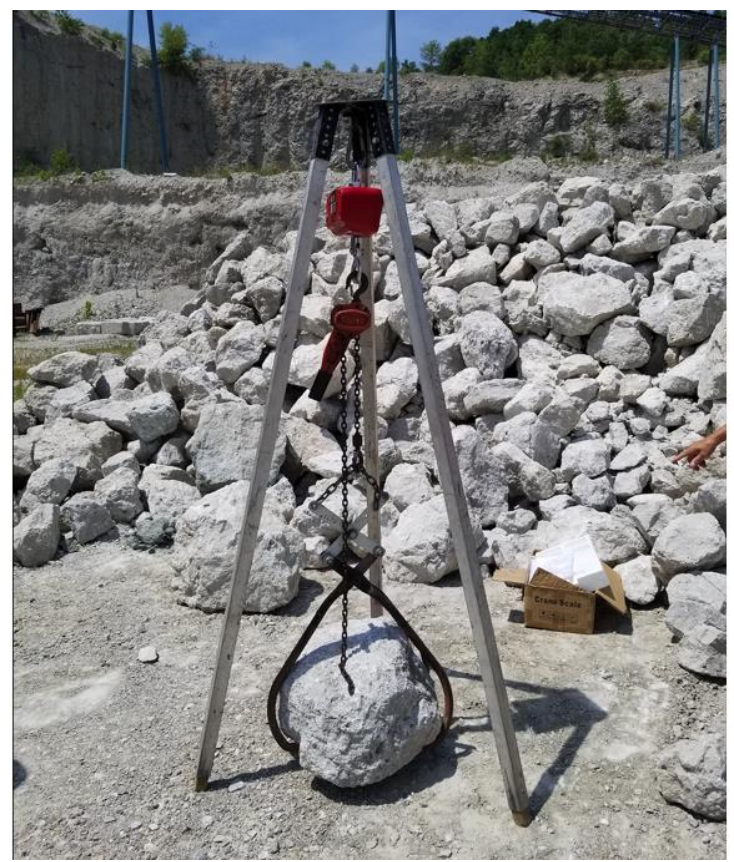

(b)

Figure 3.5. (a) Hand measurements of riprap dimensions and (b) tripod scale system used by IDOT for measuring the weight of large riprap rocks.

\subsubsection{Riprap Stockpile Image-acquisition Procedure}

For the imaging of riprap stockpiles, conventional smartphone cameras were used to obtain the images. Ideally, multiple views of the same stockpiles were taken with a calibration ball and with the camera positioned parallel to the slope of the stockpile. The following guidelines/procedures were closely followed for imaging riprap stockpiles:

- The camera requirement for acquiring an image with a sufficient resolution is $2400 \times 3000$ or higher. Most smartphone cameras will pass this requirement. 
- Stockpile images were taken from a nearly perpendicular direction against the stockpile surface, as illustrated in Figure 3.6(a).

- The stockpile images were taken from a location close to the stockpile so that most of the images were filled with useful rock pixels, and the calibration ball (if present) would not appear too small in the image. Images with and without a calibration ball were taken for the purposes of developing a robust algorithm, particularly for training and segmentation purposes. In Figure 3.6, images (b) and (c) are satisfactory images because they are taken at a close distance, the ball is at the center of the image (c), all image pixels in the image contain a rock, and the view is perpendicular to the stockpile face. Image (d) is less satisfactory because it was taken from a far distance and the camera was not perpendicular to the sloped surface.

- For all images with a calibration ball, the location of the ball was approximately at the center of the image so that the distortion effect is minimized.

- Riprap size groups from RR3 to RR7 categories were all imaged. Additionally, rocks with special colors/textures were selected, as they contributed to the robustness of the dataset.

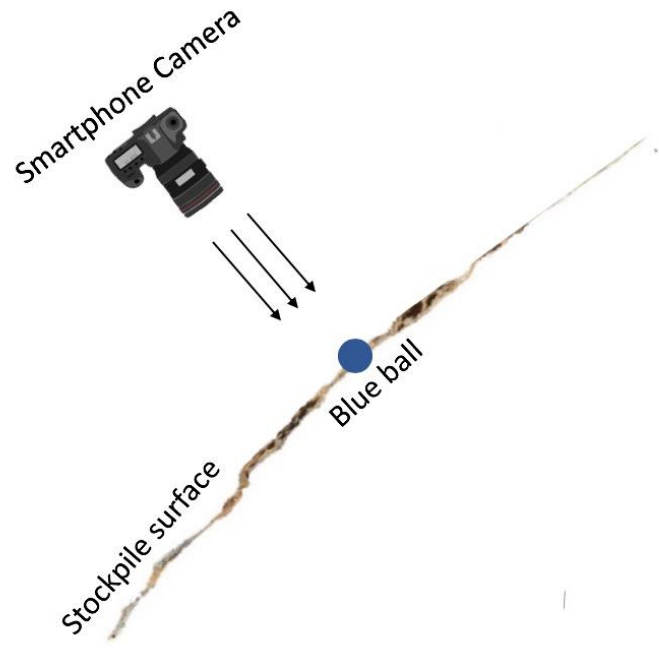

(a)

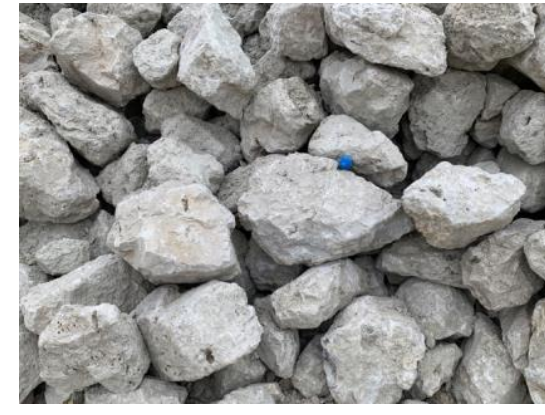

(b)

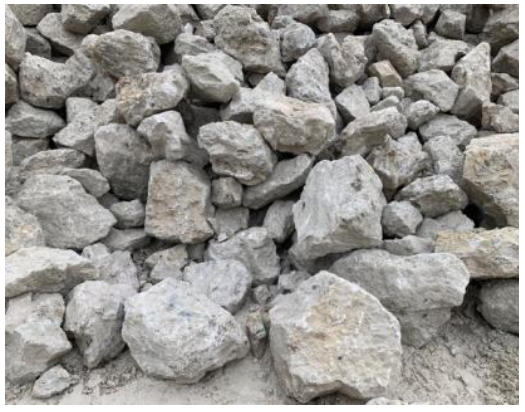

(c)

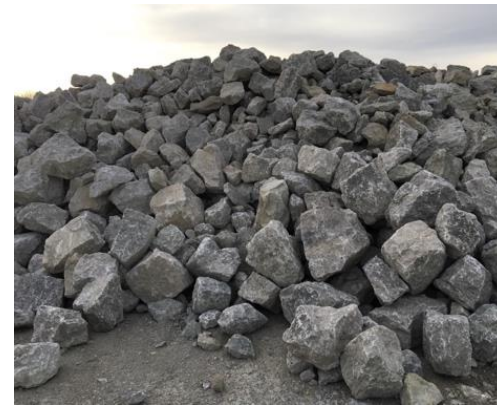

(d)

Figure 3.6. (a) Proper positioning of the camera relative to the stockpile, (b) satisfactory image with a calibration ball, (c) satisfactory image without a calibration ball, and (d) unsatisfactory image of a stockpile. 


\subsection{SUMMARY}

This chapter presented an overview of the field studies and sampling procedures of riprap material in quarries. First, IDOT size categories for riprap and lists of approved riprap sources were identified. Information for the sources selected to image single riprap particles and riprap stockpiles for this project were presented. The development of a riprap inspection system to image and segment individual rocks was detailed, along with the laboratory and field measurements to verify the accuracy of the segmentation results for images taken with the inspection system. Lastly, a procedure for imaging riprap stockpiles was presented. The following two chapters will present detailed results for the segmentation of images with single rocks and with riprap stockpiles. 


\section{CHAPTER 4: SINGLE-PARTICLE VOLUMETRIC ESTIMATION USING COMPUTER VISION}

\subsection{INTRODUCTION}

This chapter describes a volumetric estimation approach developed for the single-particle study. A computer vision-based image-segmentation algorithm was developed for extracting object information while also reducing effects of sunlight and shadowing. Based on multi-view information from the image-segmentation algorithm, a 3D reconstruction algorithm was then integrated for quantifying the volumetric properties of objects. Both algorithms are designed with minimal user input required during image-processing stages for ease in implementation and practical use. The scope of this paper establishes the relevant algorithms needed for field imaging and volumetric reconstruction of riprap and large-sized aggregates. The image analysis results are validated against ground-truth measurements. The full development of this field-inspection system is intended to be portable, affordable, and convenient for data acquisition, with reliable and efficient image-processing algorithms.

\subsection{COLOR-BASED IMAGE-SEGMENTATION ALGORITHM FOR OBJECT DETECTION}

Given an image of individual or multiple rocks under uncontrolled field lighting conditions, the first and foremost task is to accurately recognize and extract the region that comprises the objects. From the perspective of digital image processing in computer vision, this includes partitioning and registering the image pixels into either a foreground object or a background scene, which is often referred to as image segmentation (Gonzalez and Woods 2006). Because color can provide valuable information for the human eye and machine vision systems, color-based image-segmentation techniques are widely used in object detection and recognition. Hence, a color-based imagesegmentation algorithm is developed herein for the reliable and accurate extraction of rock particles from field images. The developed image-segmentation scheme involves color-space representation, foreground-background contrast enhancement, adaptive thresholding, and morphological de-noising. The field-inspection system described in Chapter 3 was used to capture images of single large-sized rocks to develop and test the accuracy of the segmentation algorithm.

\subsubsection{Color Representation Using CIE L*a*b* Space}

In trichromatic theory, color is perceived by humans as a combination of red, green, and blue, i.e., three primary colors. Along with the development of digital image technology, several 3D color spaces are proposed to represent the colors. The most popular color space is Red-Green-Blue (RGB), which is used in nearly all digital camera devices and display screens, where each pixel is represented as coordinate $(R, G, B)$ with $R, G, B \in[0,255]$ and $R, G, B \in \mathbb{N}$. However, for the purpose of color image segmentation, RGB space is not recommended. It fails to satisfy the perceptual uniformity principle of color, namely, two colors that are perceptually similar to the human eye are not closely located in RGB space in terms of Euclidean distance (Cheng et al. 2001; Busin et al. 2008). Instead, approximately uniform Hue-Saturation-Value (HSV) space and, further, uniform International Commission of Illumination (CIE) L*a*b* space are formulated via nonlinear transformations from 
RGB space and can provide better performance in color image representation (Alata and Quintard 2009; Wang et al. 2014; Christine 2013). The conceptual schema of different color representation spaces is shown in Figure 4.1.

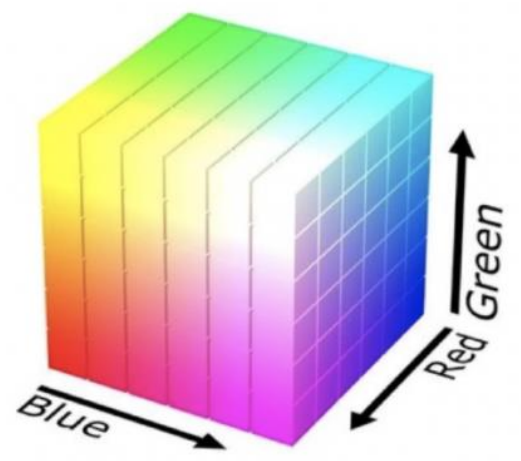

(a)

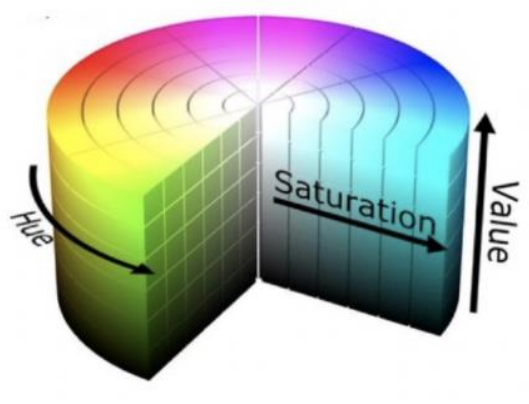

(b)

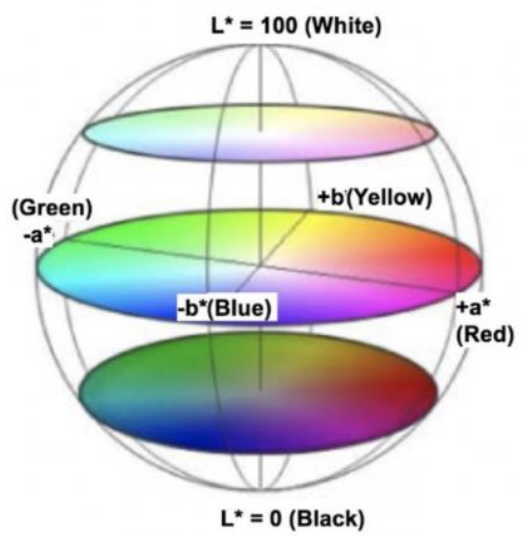

(c)

Figure 4.1. Schema of (a) RGB color space, (b) HSV color space, and (c) CIE L*a*b* color space (Kothari 2018).

In CIE L*a*b* space, the $L^{*}$ channel represents luminance or intensity value, and $a *$ and $b *$ channels track the green-to-red and blue-to-yellow transition, respectively. Particularly, it makes color chroma information less luminance-dependent, which enables effective measurement of small color differences in the shadow and highlights regions of the scene. For the original rock image presented in Figure 4.2, although an artificial blue background has been used for the convenience of segmentation, the background shadow and the large shading area on the rock surface are inevitable under field lighting conditions. Other color spaces become insufficient for object detection in these shadow regions, while $\mathrm{CIE} \mathrm{L}^{*} \mathrm{a}^{*} \mathrm{~b}^{*}$ space can properly eliminate the shadow effect by separating the useful information into its $a^{*}$ and $b^{*}$ channels. Note that the useful object information can be accumulated in $a^{*}$ channel, $b^{*}$ channel, or both, depending on what the object color and background color are. For example, when a bright-colored rock on a blue background is used (see Figure 4.2), there are few green-to-red color components in the image. Therefore, minimal object-background contrast is available in $a^{*}$ channel while most of the useful object information accumulates in $b^{*}$ channel.

The features of CIE L*a*b* space help systematically improve the robustness of the imagesegmentation algorithm under various lighting conditions. Therefore, $\mathrm{CIE} \mathrm{L}^{*} \mathrm{a}^{*} \mathrm{~b}^{*}$ is selected in this paper as the appropriate color space for the following image-segmentation process. Additionally, based on the observation that normal rock colors are rarely blue or green, selection of a background color in the blue-green zone will yield better performance in color segmentation. 


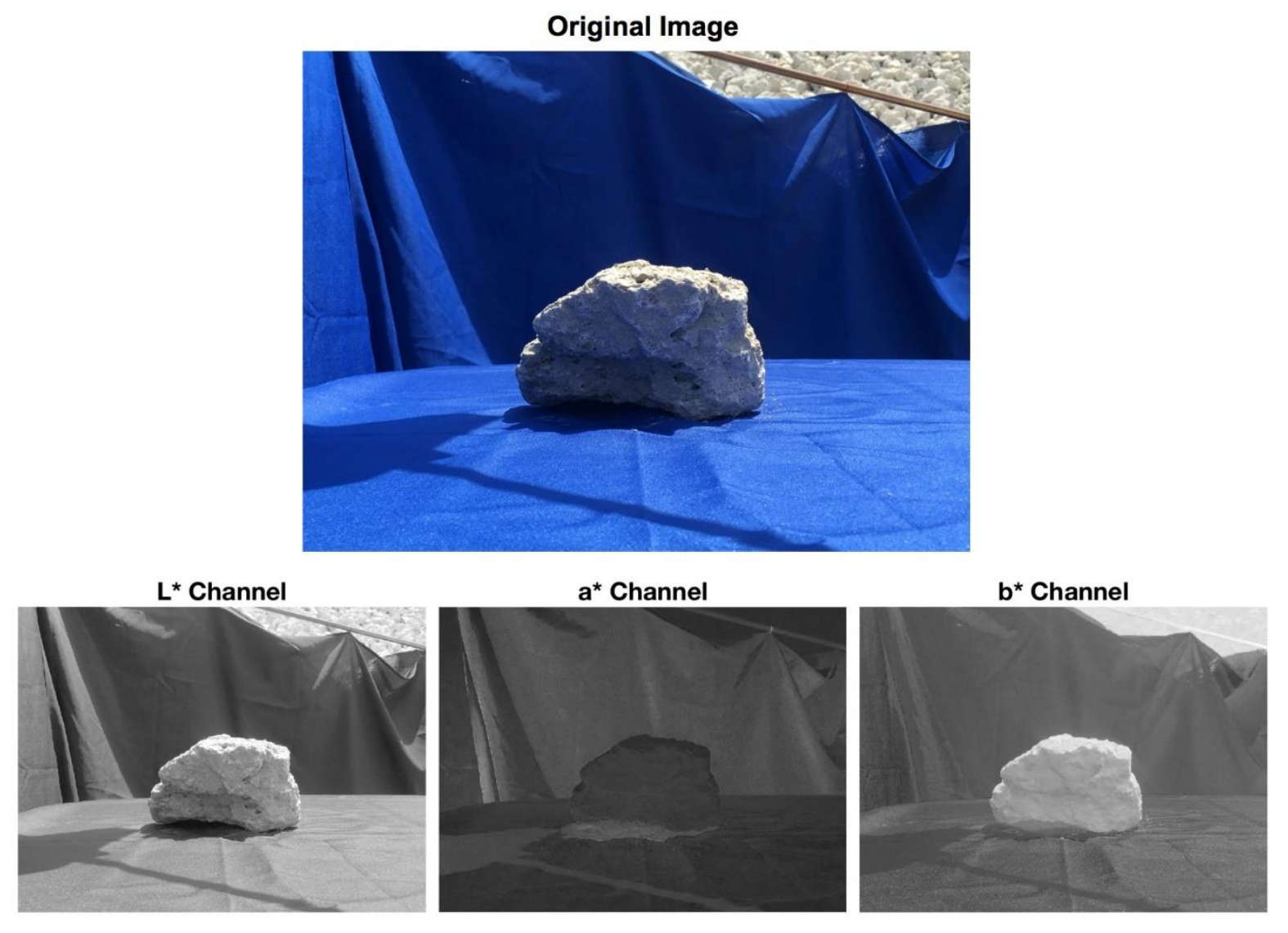

Figure 4.2. Channel images of a large riprap rock studied using CIE $L^{*} a * b *$ color space.

\subsubsection{Foreground and Background Representation Using Pixel Statistics}

To further differentiate the rock from its surroundings, the representative colors of foreground and background are calculated based on pixel statistics. As an example, the $b^{*}$ channel image in Figure 4.2 (bottom-right image) is analyzed. For the reader's convenience, $a^{*}$ and $b^{*}$ channels are denoted as the "color channel" in the following context, as compared to the $L^{*}$ channel, which is the "intensity channel."

A pixel-wise histogram is first obtained for the color channel, as shown in Figure 4.3(a). However, the histogram is comprised of discrete pixel counts, where the representative color values of foreground and background pixels can be visually defined rather than consistently quantified. Therefore, a Cumulative Distribution Function (CDF) that allows numerically characterizing pixel statistics from a continuous curve is calculated based on the pixel histogram, as shown in Figure 4.3(b). Note that the horizontal axis is the pixel value of color channel after scaling into a range $[0,1]$.

Figure 4.3 shows that when a significant number of pixels cluster around the peaks in the histogram, an increasing slope is associated in the CDF. A turning-point detection algorithm proposed in signal processing (Killick et al. 2012) is then used to capture abrupt changes in the CDF. As illustrated in Figure $4.3(\mathrm{~b})$, representative background and foreground colors $b_{\text {background }}^{*}=0.40, b_{\text {foreground }}^{*}=$ 0.89 are detected based on the pixel statistics of the $b^{*}$ channel image in Figure 4.2. 


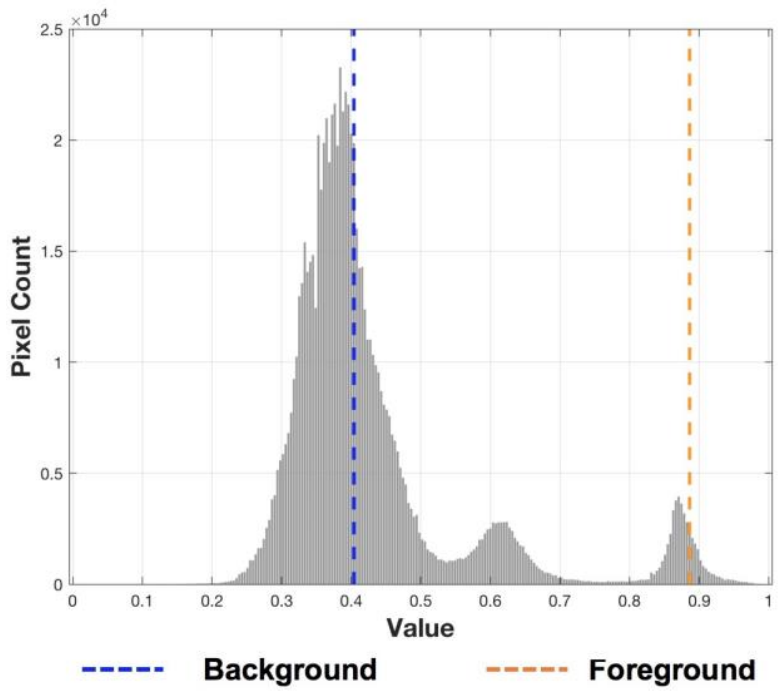

(a)

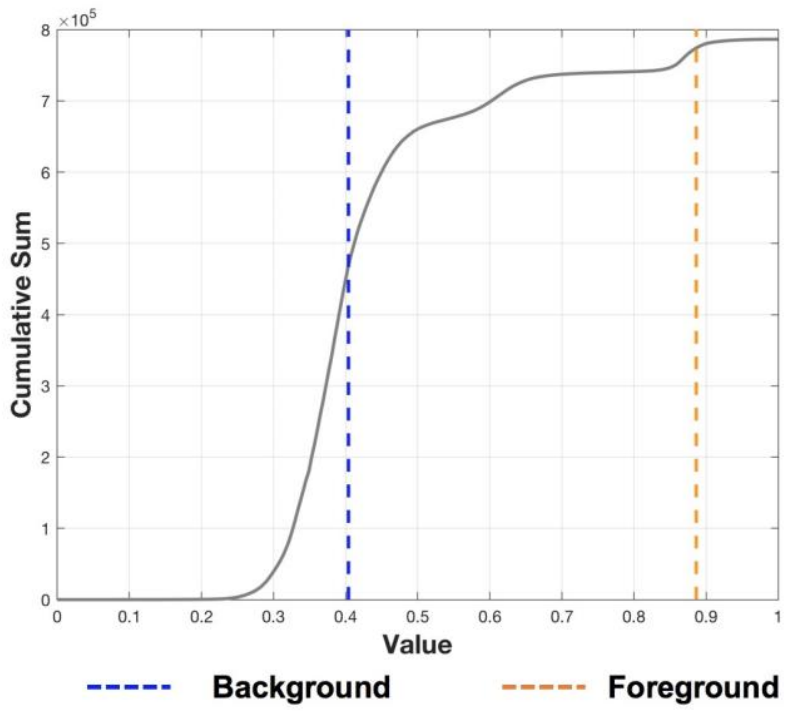

(b)

Figure 4.3. (a) Pixel histogram of color channel and (b) pixel cumulative distribution function of color channel.

\subsubsection{Contrast Enhancement Based on Color Distance}

With the representative colors of the background scene and foreground object, a color difference measure can be designed based on Minkowski distance in color space. Suppose two pixel-color vectors in the $2 \mathrm{D} a^{*}-b^{*}$ space

$$
\boldsymbol{p}_{1}=\left(a_{1}^{*}, b_{1}^{*}\right), \boldsymbol{p}_{2}=\left(a_{2}^{*}, b_{2}^{*}\right)
$$

then the Minkowski distance of order $\mathrm{p}$ (i.e., p-norm distance) between the two colors is

$$
\left\|\boldsymbol{p}_{\mathbf{1}}-\boldsymbol{p}_{\mathbf{2}}\right\|_{p}=\left(\left|a_{1}^{*}-a_{2}^{*}\right|^{p}+\left|b_{1}^{*}-b_{2}^{*}\right|^{p}\right)^{1 / p}
$$

Considering the principle of gamma correction that can bring more contrast between the object and background, the proposed color distance to be calculated at each pixel location is revised from Equation (4.2) and presented in the following generalized form:

$$
d\left(\boldsymbol{p}, \boldsymbol{p}_{\mathbf{0}}\right)=\left|a^{*}-a_{0}^{*}\right|^{\gamma}+\left|b^{*}-b_{0}^{*}\right|^{\gamma}
$$

where

$\boldsymbol{p}$ is the color $\left(a^{*}, b^{*}\right)$ at the current pixel location.

$\boldsymbol{p}_{\mathbf{0}}$ is the reference color $\left(a_{0}^{*}, b_{0}^{*}\right)$ obtained from the pixel statistics (either background or foreground representative color value can be selected as reference).

$\gamma$ is the gamma correction coefficient. $\gamma>1$ is used to contrast the object with the background, typically 2.0 (squared-Euclidean distance). 
Figure 4.4 illustrates the effectiveness of background and foreground contrast enhancement using the proposed color distance approach. In Figure 4.4(b), the pixel grayscale intensity represents the magnitude of the color distance with the foreground's representative color as the reference. Therefore, the closer the pixel color to the foreground representative color, the smaller the color distance and the darker the intensity in the distance map, and vice versa. Note that the measure of color distance helps better contrast the background and foreground and further eliminate the shadow effect.

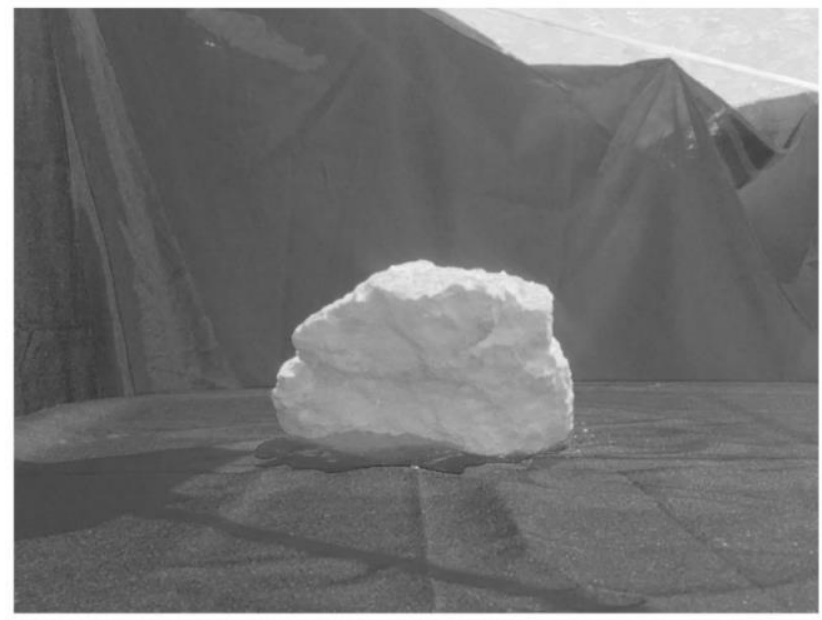

(a)

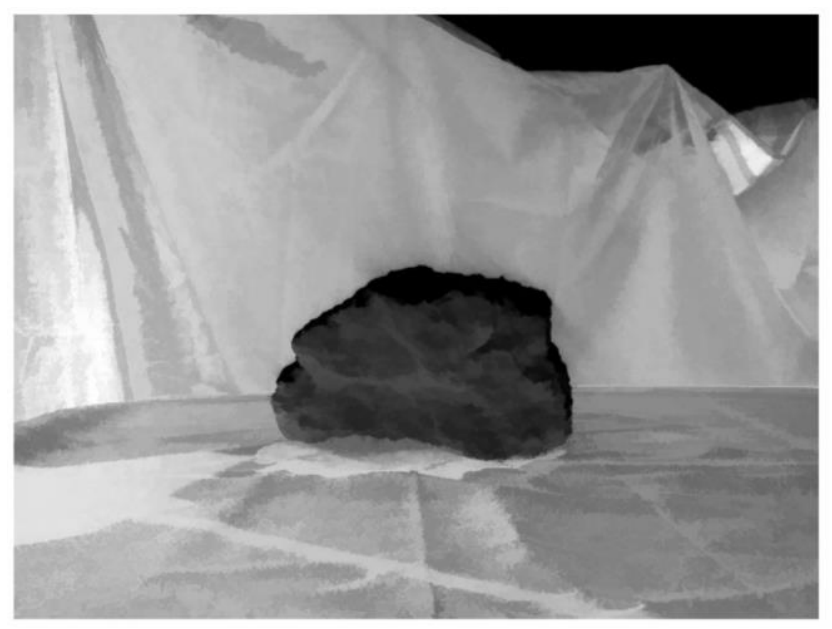

(b)

Figure 4.4. (a) Original $b^{*}$ channel image and (b) distance map with foreground representative color as the reference.

\subsubsection{Adaptive Thresholding and Morphological De-noising}

Based on the enhanced distance map, image thresholding (or binarization) is applied and a binary image can be obtained as follows:

$$
\text { Pixel value }= \begin{cases}1(\text { white }), & \text { if } v \leq v_{\text {threshold }} \\ 0(\text { black }), & \text { if } v>v_{\text {threshold }}\end{cases}
$$

The thresholding algorithm can either follow a fixed threshold value (user-defined or computed based on Otsu's bimodal method [1979]) or a flexible threshold value known as the adaptive thresholding method (Bradley et al. 2007). Because digital images are discretized by pixel, it is common that a binary image can include a significant amount of noise pixels, as shown in Figure 4.5(a). De-noising is then required to clean the noise pixels as well as complement discontinuities along the object's boundary. A series of image morphological operations are applied on the binary image, including image erosion, dilation, hole filling, etc. In addition, regions that are closer to the image border are removed, because they are often unidentified objects such as equipment or field surroundings. Figure 4.5(b) shows the binary image after morphological de-noising, and Figure 4.5(c) illustrates the object boundary detected by the image-segmentation algorithm. The detected and visualized object boundary is accurate under the intervention of strong shadows, surface reflection, unidentified objects, and so on. Furthermore, based on experiments throughout the development of 
this algorithm under both laboratory and field conditions, the robustness and versatility of the proposed image-segmentation algorithm are verified.

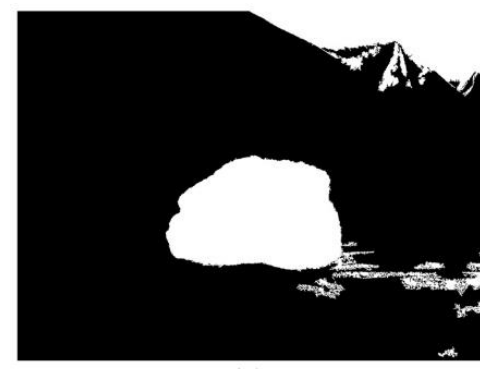

(a)

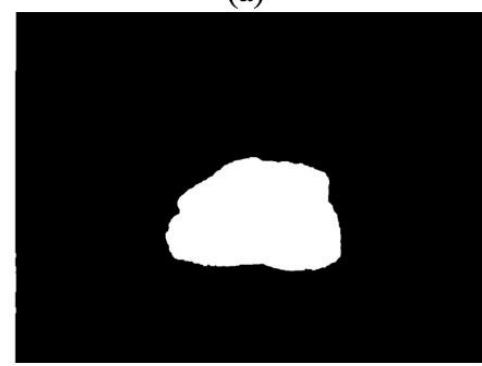

(b)

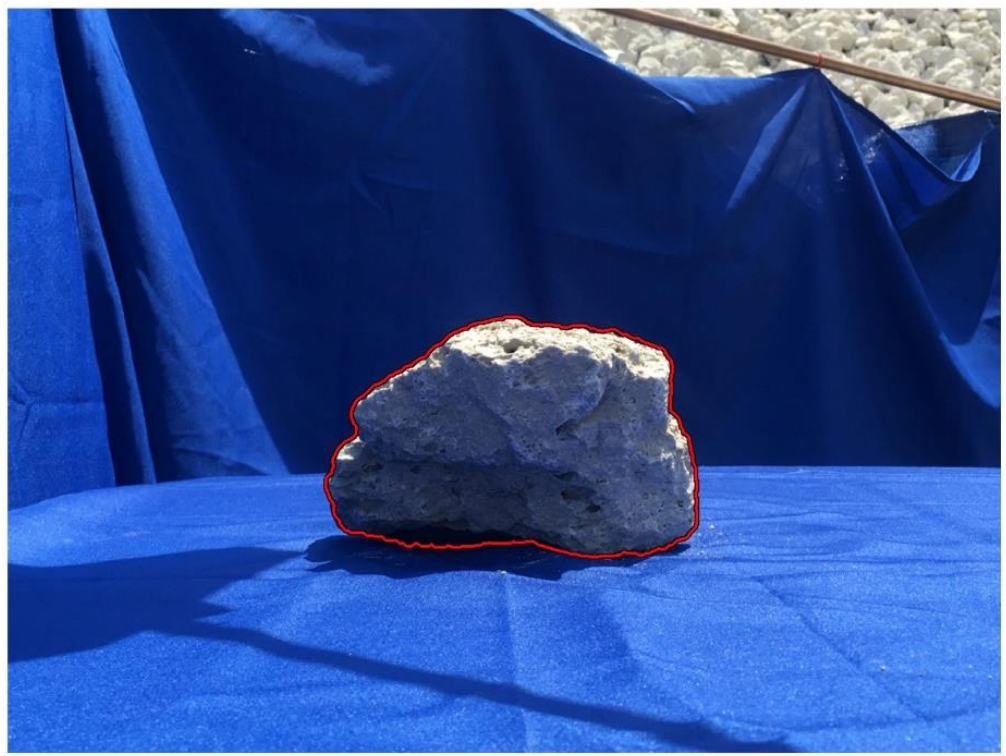

(c)

Figure 4.5. (a) Adaptive thresholding applied image, (b) morphological de-noising applied image, and (c) image-segmentation result.

\subsection{D VOLUMETRIC RECONSTRUCTION ALGORITHM}

For the volumetric reconstruction of a 3D object from 2D images, three orthogonal and equaldistance views are required. The orthogonality of views can be well controlled in a laboratory via precise camera positioning and distance measurement. However, in the field, neither ideal criteria can be easily achieved without time-consuming and lengthy efforts. To efficiently estimate the volume with sufficient precision, a 3D volumetric reconstruction algorithm using orthogonality calibration and volume correction is proposed herein.

\subsubsection{Image Resizing Based on Calibration Ball Reference}

A calibration ball is commonly used as a standard reference object to facilitate the volume/size estimation. Two options are available for using the calibration ball: 1) if the location of camera/smartphone is fixed during image acquisition, a calibration ball can be first captured before any object; and 2) if the location of camera/smartphone keeps changing or only a limited number of devices are available, the calibration ball should be captured together with the object in every image for a consistent reference. The first option usually provides more efficiency, but the latter is more versatile if the ideal condition cannot be achieved. In each case, the user is expected to take three approximately orthogonal views of the object, i.e., from the top, front, and side.

After a successful image segmentation, three silhouettes of an individual rock, ock $_{i}$, are cropped from the corresponding binary images. Accordingly, three silhouettes of the calibration ball, Ball $_{i}$, are also cropped, and their equivalent diameter $r_{i}$ are measured. The information can be paired as 
Rock $_{i}-$ Ball $_{i}-r_{i}, i=1$ (top), 2 (front), 3 (side), as shown in Figure 4.6. The three rock silhouette images $\operatorname{Rock}_{i}$ are then resized based on the equivalent diameter $r_{i}$ of its calibration ball correspondence. The purpose of this step is to counteract the effect of different lens zoom and unequal camera-object distances.

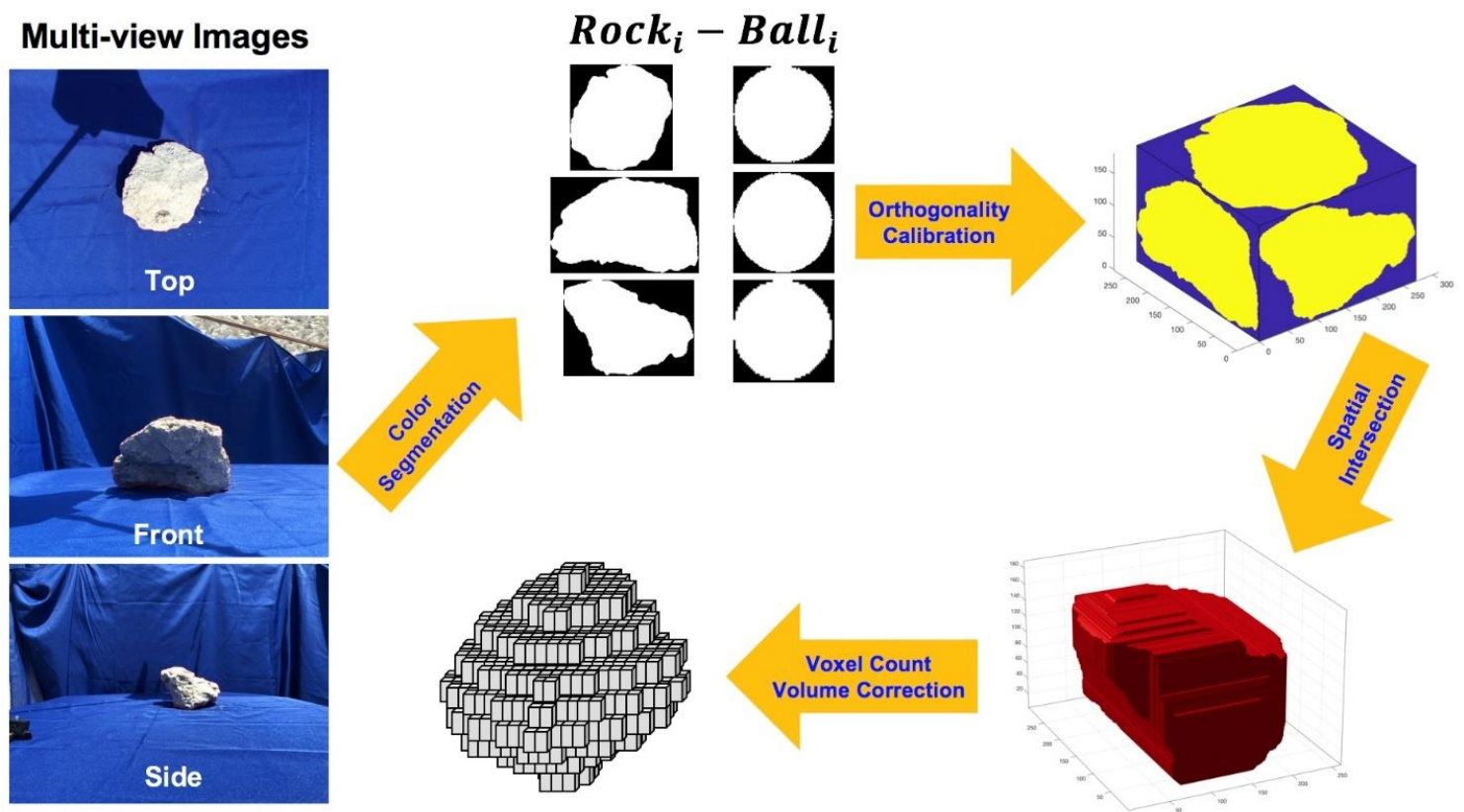

Figure 4.6. Flowchart of 3D volumetric reconstruction algorithm.

\subsubsection{Orthogonality Calibration Using Least Squares Solution}

Although the rock silhouettes have been resized with respect to the calibration ball, the dimension of these silhouettes can rarely achieve an exact match primarily because both lack perfect orthogonality and photogrammetry error. Therefore, a linear system of equations is formed as follows to obtain a standardized dimension for orthogonality correction:

Suppose the target orthogonal dimension is $\left[x_{0} \times y_{0} \times z_{0}\right]$ and each silhouette has an image heightwidth dimension of $\left[h_{\text {top }} \times w_{\text {top }}\right],\left[h_{\text {front }} \times w_{\text {front }}\right],\left[h_{\text {side }} \times w_{\text {side }}\right]$. By aligning each silhouette dimension with the orthogonal dimension (see Figure 4.6 above), the following linear system of equations should be satisfied:

$$
\text { A } \boldsymbol{x}=\boldsymbol{b}
$$

where

$$
\mathrm{A}=\left[\begin{array}{lll}
0 & 0 & 1 \\
1 & 0 & 0 \\
1 & 0 & 0 \\
0 & 1 & 0 \\
0 & 0 & 1 \\
0 & 1 & 0
\end{array}\right], \boldsymbol{x}=\left[\begin{array}{c}
x_{0} \\
y_{0} \\
z_{0}
\end{array}\right], \boldsymbol{b}=\left[\begin{array}{c}
w_{\text {top }} \\
h_{\text {top }} \\
w_{\text {front }} \\
h_{\text {front }} \\
w_{\text {side }} \\
h_{\text {side }}
\end{array}\right]
$$


The linear system in Equation (4.5) can be solved as a least squares problem to minimize the residual error term, i.e.:

$$
\begin{gathered}
\boldsymbol{x}_{*}=\underset{\boldsymbol{x}}{\arg \min _{\boldsymbol{1}}}\|\mathrm{A} \boldsymbol{x}-\boldsymbol{b}\|_{2}^{2} \\
\Uparrow \\
\mathrm{A}^{T} \mathrm{~A} \boldsymbol{x}_{*}=\mathrm{A}^{T} \boldsymbol{b}
\end{gathered}
$$

where the target orthogonal dimension $\boldsymbol{x}_{*}$ is obtained by solving the normal Equation (4.6).

\subsubsection{Spatial Intersection of Multi-View Silhouettes}

Three silhouettes are then calibrated to the orthogonal dimension $\boldsymbol{x}_{*}=\left[x_{0} \times y_{0} \times z_{0}\right]$, and the object solid can now be reconstructed by replicating each binary silhouette along its orthogonal dimension and determining their intersection set, as shown in Figure 4.6 above. The intersection set is represented as a binary matrix where the object solid has a value of 1 . The volume of the intersected body can then be calculated in terms of "voxels," namely a 3D cuboid version of pixel. The reconstructed volume can be obtained from the voxel ratio between the rock and calibration ball.

\subsubsection{Volume Correction}

Note that the reconstruction algorithm based on orthogonal views will always overestimate the volume of the object (Rao 2001). The overestimation mainly results from the following two aspects: systematic overestimation, which is related to the algorithm methodology, and image resolutionbased overestimation, which is limited by the image precision. A detailed analysis is conducted on both aspects, and corresponding corrections are applied to the reconstructed volume result.

\subsubsection{Systematic Correction}

Denoting the actual voxel set of the object as $S$ and the reconstructed one as $S^{\prime}$, then the volume (total number of voxels) of the reconstructed object, $V\left(S^{\prime}\right)$, should always be equal to or greater than the volume of the actual object, $V(S)$ :

$$
V(S) \leq V\left(S^{\prime}\right)
$$

Equation (4.7) can be proven by contradiction as follows:

The reconstructed object $S^{\prime}$ must share an identical silhouette with the actual object $S$ from three orthogonal views, i.e., the following statement must hold during the reconstruction process

$$
\pi_{i}(S)=\pi_{i}\left(S^{\prime}\right)=s_{i}(i=1,2,3)
$$

where

$\pi_{i}$ is the silhouette-projection operation along direction I.

$S_{i}$ is the projected silhouette along direction $\mathrm{i}$. 
Suppose the proposition in Equation (4.7) is false, then $V(S)>V\left(S^{\prime}\right)$. Accordingly, there must be a voxel $M$ in $S$ but not in $S^{\prime}$, i.e., $\exists M(x, y, z), M \in S, M \notin S^{\prime}$. This implies that at least one of the three silhouette projections of $M$ does not lie within the silhouettes of both $S$ and $S^{\prime}$, i.e.

$$
\exists i \in\{1,2,3\}, \pi_{i}(M) \in \pi_{i}(S), \pi_{i}(M) \notin \pi_{i}\left(S^{\prime}\right) \Rightarrow \pi_{i}(S) \neq \pi_{i}\left(S^{\prime}\right)
$$

which contradicts the statement in Equation (4.8). By contradiction, Equation (4.7) is proven.

However, this systematic overestimation is hard to measure quantitatively for two reasons. The first is the high randomness of the riprap shape, which is a natural property of riprap based on the productive process. The second is the insufficient surficial information on dents, hollow portions, or cavities that are not in sight of cameras (Rao 2011). Therefore, a correction factor $c_{1}$ for eliminating the systematic overestimation can only be selected empirically. Based on preliminary laboratory data, $c_{1}=0.95$ is reckoned and used in the volume correction process.

\subsubsection{Resolution-based Correction}

As the first step in the image-segmentation algorithm, images are compressed into $w \times h$ ( $w \geq h$, typically $1024 \times 768$ ) resolution. In a digital image, there is usually a pixel-wise transition from an object to the background. Because the segmentation algorithm is based on foreground-background contrast, the detected boundary will slightly shrink from the actual object boundary. Typically, a onepixel difference can be observed between the detected boundary and actual boundary, as illustrated in Figure 4.7.

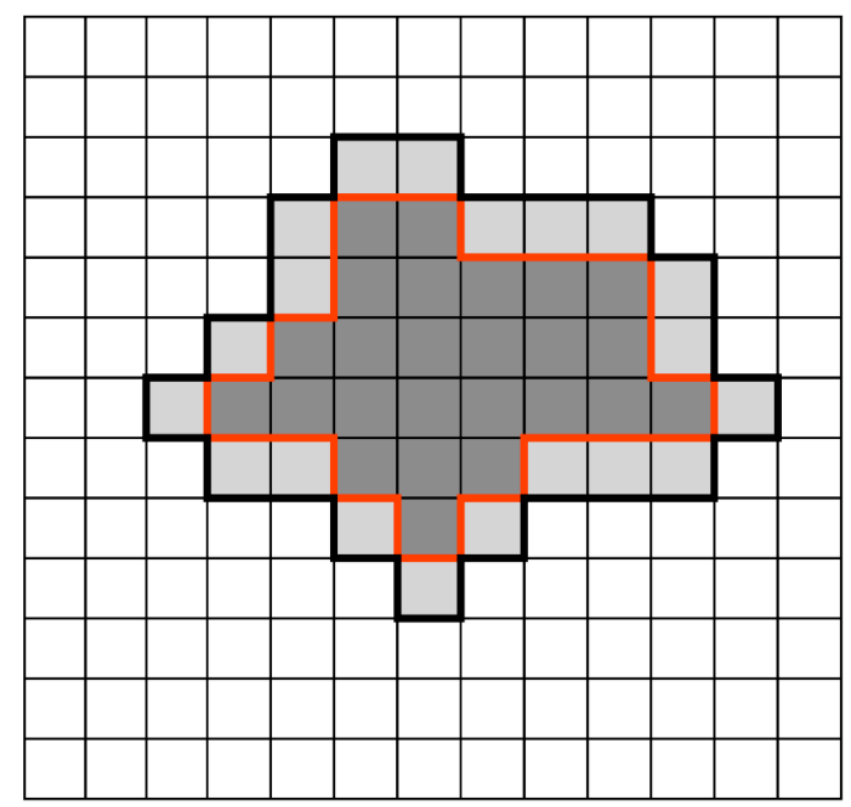

— : Detected boundary

- : Actual boundary

Figure 4.7. Pixel-scope difference between the detected boundary and actual boundary. 
This effect can cause resolution-based overestimation controlled by two factors. The first one is the relative size ratio between the rock and calibration ball, and the second is the absolute pixel occupancy of the calibration ball. The influence of these two factors is presented as follows.

For the convenience of analysis and explanation, sphere-shaped objects are assumed for both the calibration ball and riprap rock. Suppose the actual radii of the calibration ball and rock are $r_{\text {ball }}$ and $r_{\text {rock }}$, respectively. Based on the observation in Figure 4.7, their detected radii in image will be $r_{b a l l}-$ 1 and $r_{\text {rock }}-1$, both in terms of pixel. Denote the volume of reconstructed ball as $V_{\text {ball }}$ and the volume of reconstructed rock (based on detected boundary before applying any volume correction) as $V_{\text {rock_detected }}$, both in terms of voxels. Then, the ratio between the reconstructed rock's volume and the reconstructed ball's volume is given as follows:

$$
\frac{V_{\text {rock_detected }}}{V_{\text {ball }}}=\frac{\left(r_{\text {rock }}-1\right)^{3}}{\left(r_{\text {ball }}-1\right)^{3}}
$$

The actual volume of rock is denoted as $V_{\text {rock }}$ in terms of voxels. The ratio of the actual rock's volume and the actual ball's volume is given as follows:

$$
\frac{V_{\text {rock }}}{V_{\text {ball }}}=\frac{r_{\text {rock }}^{3}}{r_{\text {ball }}^{3}}
$$

Then, the resolution-based correction factor $c_{2}$ is calculated by:

$$
c_{2}=\frac{V_{\text {rock }}}{V_{\text {rock_detected }}}=\frac{\left(r_{\text {ball }}-1\right)^{3} r_{\text {rock }}^{3}}{\left(r_{\text {rock }}-1\right)^{3} r_{\text {ball }}^{3}}
$$

Let $t=\frac{r_{\text {rock }}}{r_{\text {ball }}}$, then Equation (4.12) can be simplified into:

$$
c_{2}=\left(1-\frac{t-1}{t \cdot r_{\text {ball }}-1}\right)^{3}
$$

The correction factor $c_{2}$ is a function of the relative size ratio $t$ and the absolute pixel occupancy $r_{\text {ball }}$, as shown in Figure 4.8. Note that with the increase of $t$, or decrease of $r_{b a l l}$, the value of resolutionbased correction factor $c_{2}$ will decrease. To better illustrate the effect, typical values of $r_{b a l l}=$ $\{45,25,15\}$ and $t \in[1,15]$ are selected for a parametric analysis. For example, in a $1024 \times 768$ image, when the calibration ball has a radius of 25 pixels and the relative size ratio between rock and ball equals 7 , the correction factor to be applied will be $c_{2}=0.90$.

As a result, correction factors $c_{1}=0.95$ for systematic correction and $c_{2}$ from Equation (4.13) for resolution-based correction will be applied to the reconstructed rock volume at the end of the reconstruction algorithm. 


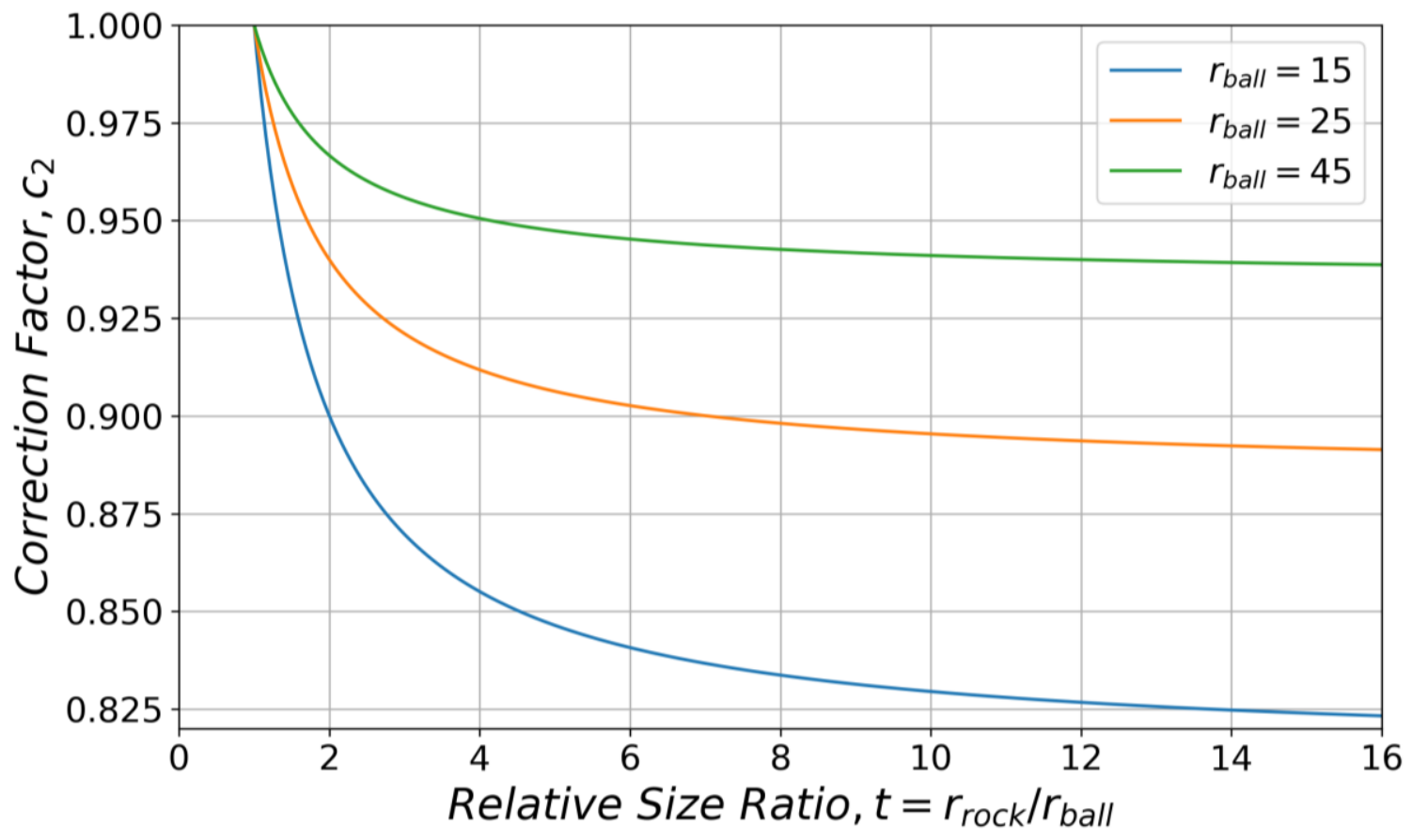

Figure 4.8. Effect of rock/ball ratio and calibration ball size on volume overestimation.

\subsection{RESULTS AND DISCUSSION}

After applying the image segmentation and 3D reconstruction algorithms to all image triplets, the reconstructed volume of each riprap rock was obtained. As described in section 3.4.1, the reconstructed results were validated with ground-truth volume/weight measurements and compared with results from IDOT's hand measurement practice.

For Source 1 particles (see Table 3.3), because the volume was directly measured during the specific gravity test, comparison was made between the reconstructed volume and measured volume. For Source 2 and Source 2-Large particles, because only the weight information was available on-site, the reconstructed volume and the volume calculated from hand measurement were first converted to weight based on a typical specific gravity value $G_{s}=2.66$ and then compared with the measured weight. The results are presented in Figure 4.9, Figure 4.10, and Table 4.1.

Figure 4.9(a) compares all reconstructed volume results (i.e., with three rotate repetitions) with the ground-truth measurements. Figure 4.9(b) compares the averaged volume results with the groundtruth measurements for Source 1 particles. Similarly, Figure 4.9(c) and Figure 4.9(d) compare the reconstructed results with repetitions and after averaging with the ground-truth measurements for Source 2 particles, but in terms of weight. A 45-degree line is plotted as the reference for the groundtruth comparisons. Error bars are used in the averaged results plot to present the standard deviation among three rotate repetitions for individual particles. For consistently quantifying the error for each source, the following statistical indicator Mean-Absolute-Percentage-Error (MAPE) was calculated as follows: 


$$
\operatorname{MAPE}(\%)=\frac{\sum_{i=1}^{N}\left|\frac{E_{i}-M_{i}}{M_{i}}\right|}{N}
$$

where

$E_{i}$ is the estimated result from image analysis or hand measurement of $\mathrm{i}^{\text {th }}$ particle.

$M_{i}$ is the ground-truth measurement of $\mathrm{i}^{\text {th }}$ particle.

$N$ is the total number of particles.

Note that the average results have less deviations from the ground-truth measurements in terms of MAPE, i.e., 3.6\% and 7.9\%, as compared to the 5.1\% and 8.1\% for Source 1 and Source 2 particles, respectively. This indicates that increasing the number of image viewing angles and averaging the results can help reduce the random sampling error by obtaining more comprehensive stereophotography information of the object. Moreover, by comparing Source 1 and Source 2 particles, it is observed that the assumption made on material specific gravity could introduce an additional source of error. Source 1 was compared by accurately measured rock volumes and thereby smaller deviation from ground truth (3.6\%) was achieved, while the assumption of $G_{s}=2.66$ made on Source 2 rocks can bring additional error to the weight comparison (7.9\%). Additionally, the main drawback of the silhouette-based 3D reconstruction approach is its inability to reconstruct concavities (Cremers and Kolev 2011). This is an inherent property of the reconstruction approach and introduces an inevitable source of error.

Figure 4.9(e) and Figure 4.9(f) show the complete database of the image analysis results with Source 1, Source 2, and Source 2-Large particles. Axis breaks are made to better visualize the results that span a large range. Note that most data points lie within $\pm 20 \%$ error band from the reference line, and more than half of them locate within the $\pm 10 \%$ band, for all the 85 particles. Good agreement is achieved between the image analysis results and ground-truth measurements, in terms of either accurately measured volume or converted weight with given specific gravity. 


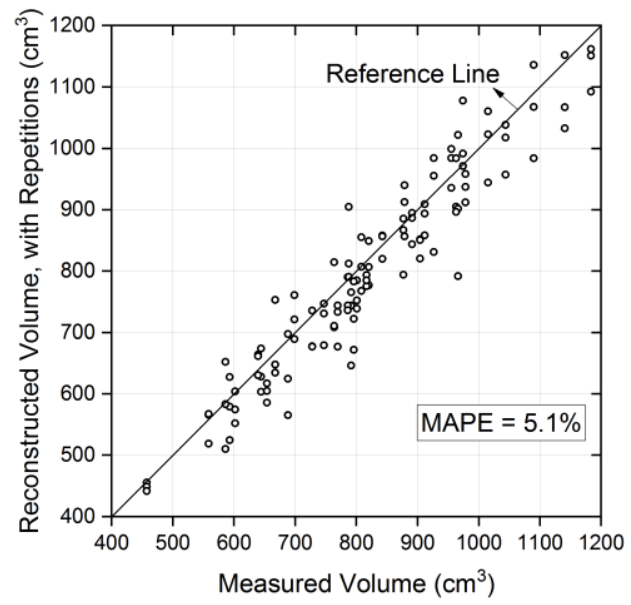

(a)

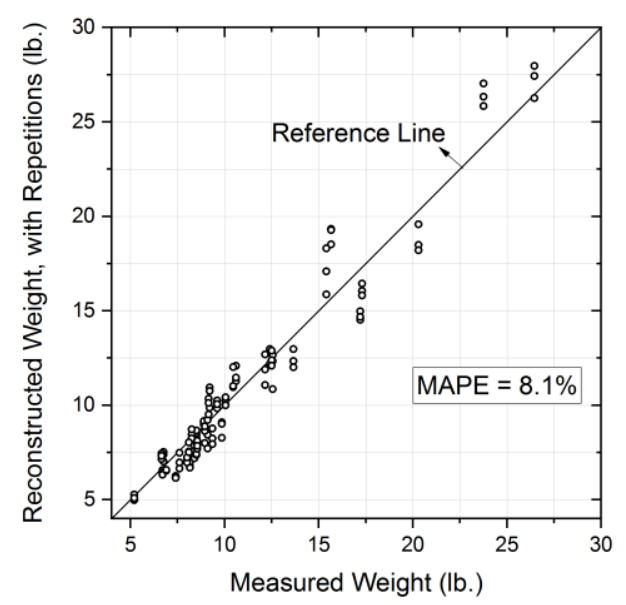

(c)

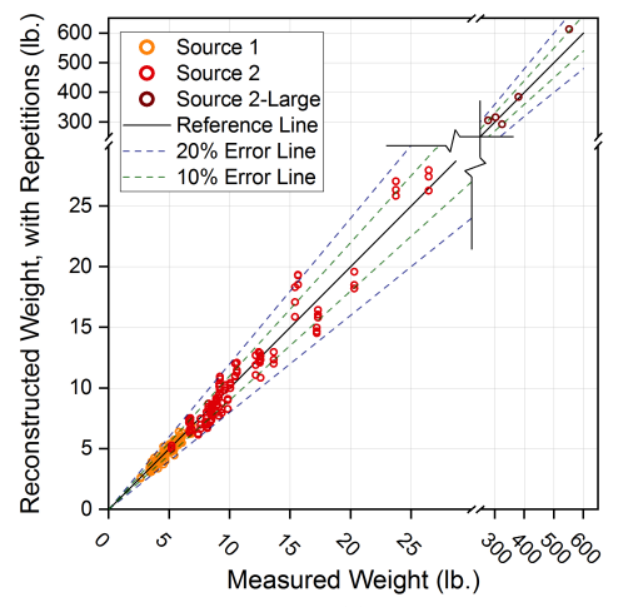

(e)

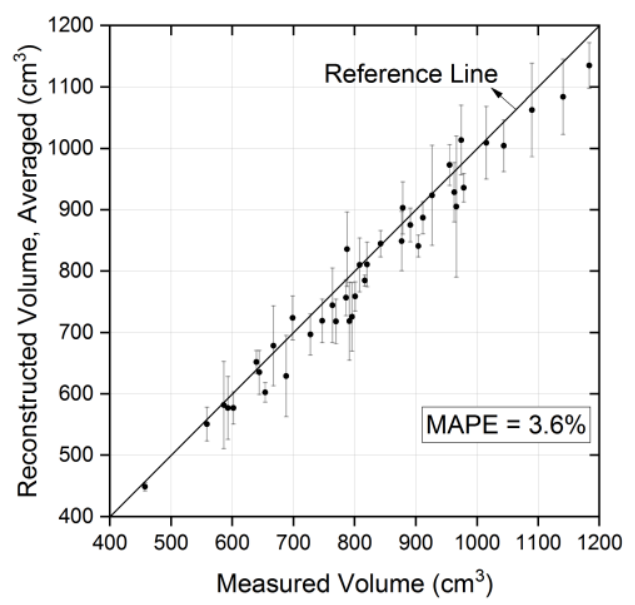

(b)

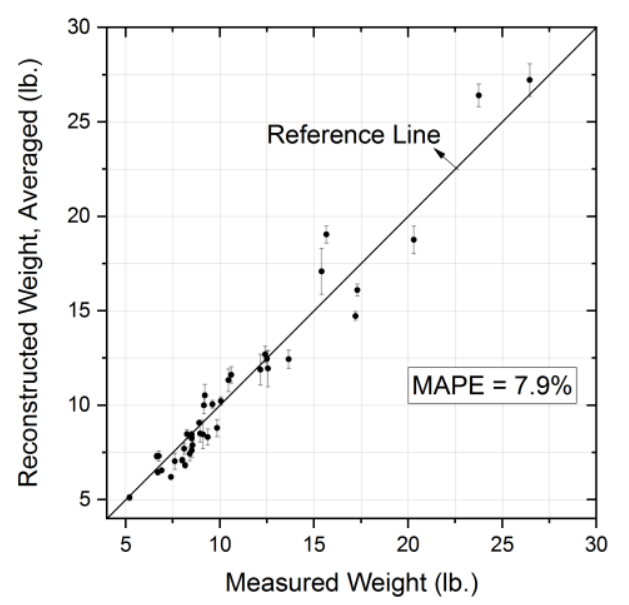

(d)

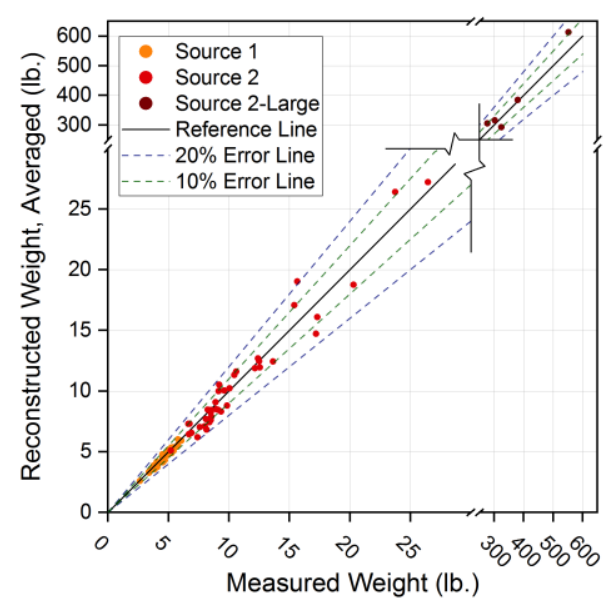

(f)

Figure 4.9. Comparisons between image analysis results and ground-truth measurements for: (a) Source 1 material with rotate repetitions, (b) Source 1 material after averaging, (c) Source 2 material with rotate repetitions, (d) Source 2 material after averaging, (e) all sources with rotate repetitions, and $(\mathrm{f})$ all sources after averaging $(1 \mathrm{lb} .=0.454 \mathrm{~kg})$. 
Table 4.1 presents the hand measurement data and image analysis results. Volume estimation procedure of the hand measurements is based on a cuboid assumption where the volume of riprap is the multiplication of three estimated midway dimensions from roughly orthogonal axes, i.e., $V=$ $a \times b \times c$. The volume results from two approaches are then converted to weight using $G_{s}=2.66$.

Table 4.1. Comparisons between Image Analysis Results and Hand Measurements on Source 2 and Source 2-Large Particles

\begin{tabular}{|c|c|c|c|c|c|}
\hline$(-)$ & $\begin{array}{l}\text { Measured } \\
\text { Weight } \\
\text { (lb.) }\end{array}$ & $\begin{array}{c}\text { Weight } \\
\text { Estimated } \\
\text { from Hand } \\
\text { Measurement } \\
\text { (lb.) }\end{array}$ & $\begin{array}{c}\text { Weight } \\
\text { Estimated } \\
\text { from Image } \\
\text { Analysis } \\
\text { (lb.) }\end{array}$ & $\begin{array}{c}\text { Error } \\
\text { Associated } \\
\text { with Hand } \\
\text { Measurement } \\
(\%) \\
\end{array}$ & $\begin{array}{c}\text { Error } \\
\text { Associated } \\
\text { with Image } \\
\text { Analysis } \\
(\%)\end{array}$ \\
\hline $2-1$ & 9.6 & 10.3 & 10.0 & 7.4 & 4.7 \\
\hline $2-2$ & 9.2 & 17.7 & 10.5 & 91.9 & 14.4 \\
\hline $2-3$ & 15.6 & 50.2 & 19.0 & 220.8 & 21.7 \\
\hline $2-4$ & 10.6 & 18.4 & 11.6 & 73.4 & 9.5 \\
\hline $2-5$ & 9.1 & 16.6 & 10.0 & 82.0 & 9.2 \\
\hline $2-6$ & 15.4 & 17.5 & 17.1 & 13.6 & 11.0 \\
\hline $2-7$ & 17.2 & 21.2 & 14.7 & 23.2 & 14.4 \\
\hline $2-8$ & 10.4 & 33.2 & 11.3 & 217.3 & 8.4 \\
\hline $2-9$ & 6.6 & 16.1 & 7.3 & 142.8 & 9.8 \\
\hline $2-10$ & 12.5 & 19.0 & 11.9 & 51.6 & 4.8 \\
\hline $2-11$ & 8.0 & 16.6 & 7.1 & 108.1 & 11.5 \\
\hline $2-12$ & 17.3 & 25.7 & 16.1 & 48.7 & 6.9 \\
\hline $2-13$ & 8.1 & 15.6 & 7.7 & 92.2 & 4.9 \\
\hline $2-14$ & 10.0 & 22.4 & 10.2 & 122.6 & 1.7 \\
\hline $2-15$ & 7.6 & 9.6 & 7.0 & 26.4 & 7.4 \\
\hline $2-16$ & 8.9 & 6.8 & 8.5 & 23.9 & 5.0 \\
\hline $2-17$ & 6.9 & 6.5 & 6.6 & 6.0 & 5.0 \\
\hline $2-18$ & 12.5 & 15.6 & 12.5 & 24.5 & 0.3 \\
\hline $2-19$ & 26.4 & 32.8 & 27.2 & 24.0 & 2.9 \\
\hline $2-20$ & 23.7 & 31.1 & 27.4 & 31.1 & 15.4 \\
\hline $2 \mathrm{~L}-1$ & 380.0 & 481.1 & 384.7 & 26.6 & 1.2 \\
\hline $2 \mathrm{~L}-2$ & 324.5 & 403.6 & 292.9 & 24.4 & 9.7 \\
\hline $2 L-3$ & 302.0 & 513.7 & 315.9 & 70.1 & 4.6 \\
\hline $2 \mathrm{~L}-4$ & 552.0 & 1034.4 & 613.3 & 87.4 & 11.1 \\
\hline $2 \mathrm{~L}-5$ & 277.0 & 461.3 & 305.3 & 66.5 & 10.2 \\
\hline MAPE (\%) & na & na & na & 68.3 & 8.2 \\
\hline
\end{tabular}

Notes: na $=$ not applicable; $(1 \mathrm{lb} .=0.454 \mathrm{~kg})$ 
Figure 4.10 shows the comparisons between hand measurements and imaging-based reconstructed results. Note that the hand measurement results have much larger errors than the imaging-based results, with MAPE $=68.3 \%$ when compared against the ground truth. Furthermore, a consistent overestimation has been observed that most of the particles fall outside the $\pm 20 \%$ error band. This can be explained because the midway dimensions are based on visual estimation and hand measurement, which have more subjectivity and higher variability. In contrast, imaging-based results have a much better accuracy with a MAPE $=8.2 \%$.

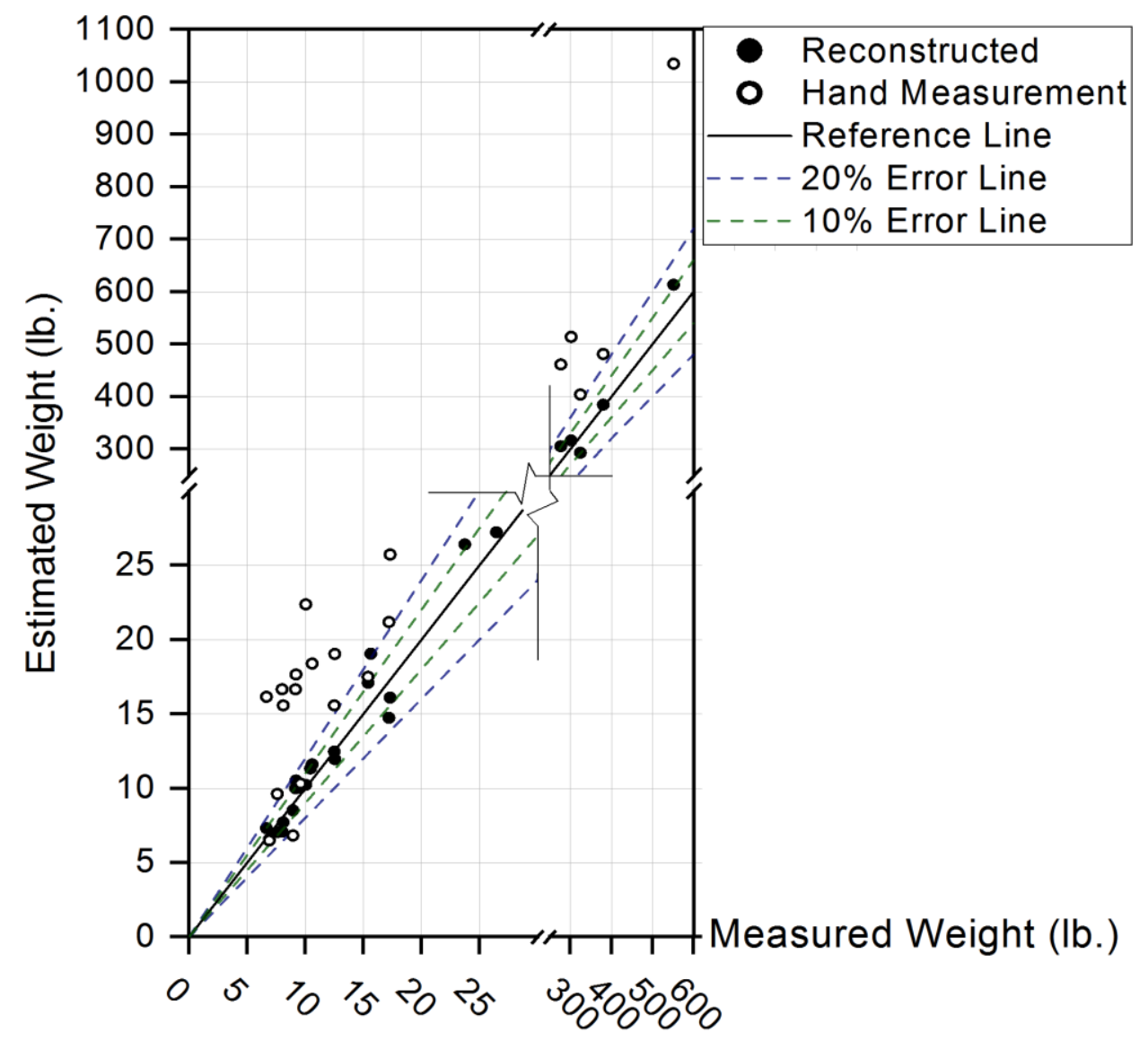

Figure 4.10. Comparisons between weights estimated from image analyses and weights estimated from hand measurements on Source 2 and Source 2 -Large particles ( $1 \mathrm{lb} .=0.454 \mathrm{~kg})$.

Overall, the consistent improvements in estimating volume/weight of medium- to large-sized particles validates the robustness and accuracy of the algorithm and shows great potential of the algorithm for further development and implementation. Moreover, the similarity between the results within three rotate repetitions implies the reproducibility of the algorithm.

\subsection{SUMMARY}

This chapter presented an innovative approach for characterizing the volumetric properties of riprap by establishing a field-imaging system associated with newly developed color image-segmentation 
and 3D reconstruction algorithms. The field-imaging system described in this chapter with its algorithms and field application examples was designed to be portable, deployable, and affordable for efficient image acquisition.

The robustness and accuracy of the image segmentation and 3D reconstruction algorithms were validated against ground-truth measurements collected in stone quarry sites and compared with state-of-the-practice inspection methods. The imaging-based results showed good agreement with the ground truth and provided improved volumetric estimation when compared to currently adopted inspection methods. Based on the results and findings, the innovative imaging-based system is envisioned for full development to provide convenient, reliable, and sustainable solutions for the onsite QA/QC tasks relating to individual riprap rocks and large-sized aggregates. 


\section{CHAPTER 5: RIPRAP STOCKPILE PARTICLE SIZE AND SHAPE ESTIMATION USING DEEP LEARNING}

\subsection{INTRODUCTION}

As compared to the single-particle imaging system developed in Chapter 4, this chapter presents an innovative approach to provide riprap stockpile image segmentation and morphological analysis. Aggregate imaging systems developed to date for size and shape characterization have primarily focused on measurement of separated or slightly non-overlapping aggregate particles. Development of efficient computer vision algorithms is urgently needed for image-based evaluations of densely stacked (or stockpile) aggregates, which requires image segmentation of a stockpile for the size and morphological properties of individual particles. Deep-learning-based techniques are utilized to achieve effective, automated, and user-independent segmentation and morphological analyses.

\subsection{DEEP-LEARNING-BASED WORKFLOW}

To analyze stockpile aggregate images, the objective is to establish an innovative approach consisting of an image-segmentation kernel based on deep-learning framework and a morphological analysis module for particle shape characterization. The flowchart of the research approach follows a preparation-training-analysis pipeline (see Figure 5.1).

The deep-learning-based image-segmentation process is data-driven and thus requires a high-quality labeled training dataset from which to extract and learn the intricate image features needed. In the preparation step, stockpile aggregate images are collected, and individual riprap rock particles in the images were manually labeled. These manual labels, or annotations, commonly serve as the groundtruth data for both training and validation purposes in image-segmentation problems. Furthermore, transfer learning is usually used as a time- and cost-saving solution in deep-learning research by utilizing generalized models that are already pretrained on a large dataset and fine-tuned to taskspecific data (Dietterich et al. 1997). Therefore, a pretrained object recognition model on the Microsoft COCO (Common Objects in Context) image dataset (Lin et al. 2014) is used in the training process together with 164 manually labeled stockpile aggregate images. Twenty additional manually labeled images are used as the validation set to measure the performance of the resulting trained model.

To train the image-segmentation kernel, a state-of-the-art deep-learning framework for object detection and segmentation, Mask R-CNN, is selected as the candidate architecture. Training parameters are tested and tuned to achieve optimal performance of the final image-segmentation kernel. Upon input of a stockpile aggregate image, the segmentation kernel performs object detection and semantic segmentation and outputs the regions of each segmented aggregate. In the analysis step, morphological analysis is conducted on each aggregate with reference to a calibration object, then collective statistics of the particle properties in the stockpile image are illustrated in the form of histogram and cumulative distribution. Completeness and precision on the validation dataset are analyzed to investigate the accuracy and robustness of the approach. 


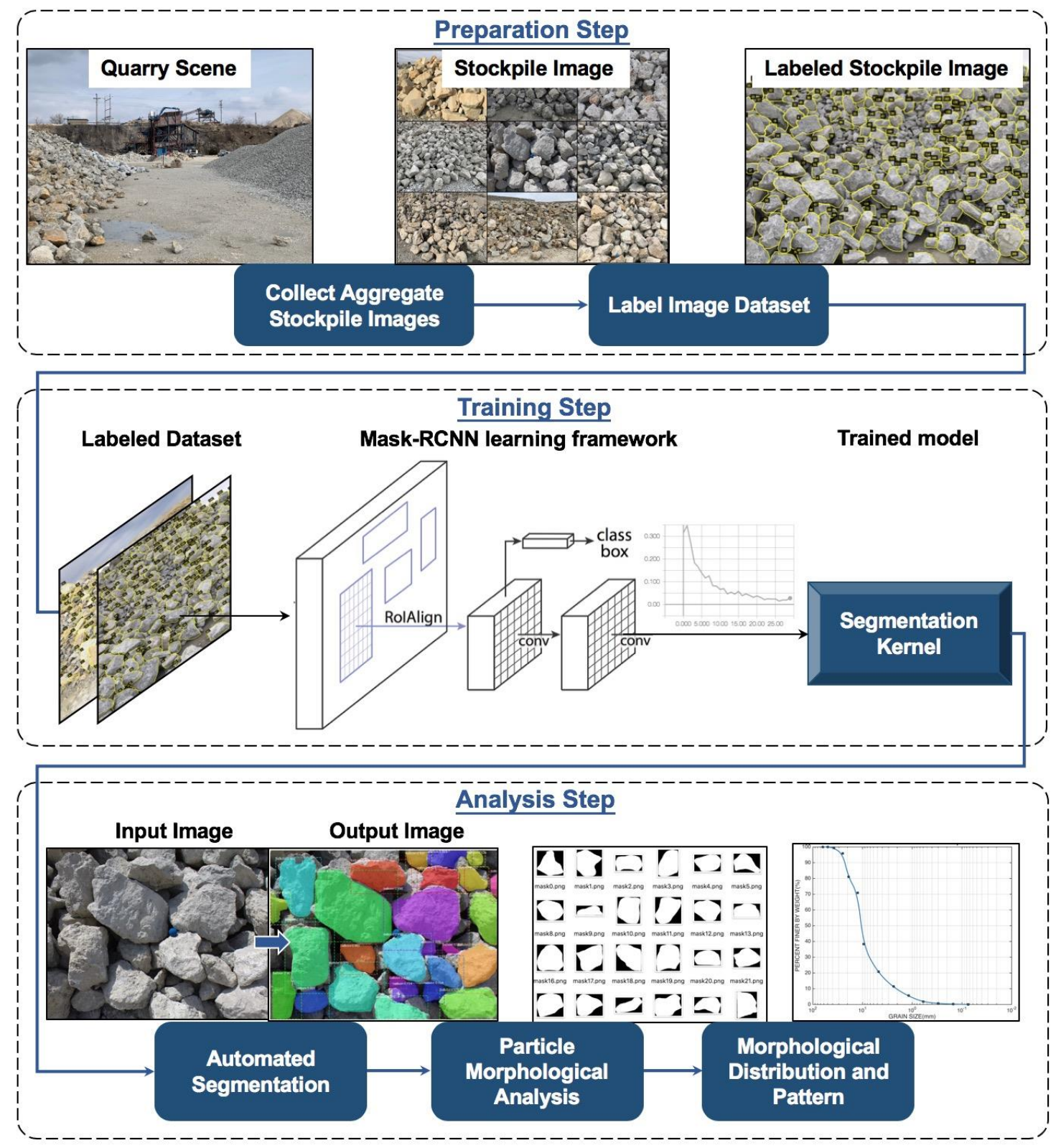

Figure 5.1. Flowchart of deep-learning-based image segmentation and morphological analysis approach.

\subsection{LABELED DATASET OF RIPRAP STOCKPILE IMAGES}

As the task-specific data for the training, stockpile aggregate images in the dataset are collected based on the following criteria: (a) the dataset should include aggregates from various geological origins and aggregate producers, and (b) the dataset should generally cover aggregates with varying size, color, texture, and from different viewing angles. Based on the source information specified in Chapter 3 (Table 3.4), the details of aggregate producer, number of images taken, and number of labeled aggregates in all images for establishing the stockpile image dataset are detailed in Table 5.1. 
Table 5.1. Source Information and Description of Stockpile Aggregate Image Dataset

Aggregate Producer

Prairie Material-Ocoya, IL

RiverStone Group, Allied Stone-Milan, IL

RiverStone Group, Midway Stone-Hillsdale, IL

Vulcan Materials Company-Kankakee, IL
Number of Images

6

14

982

100

6,766

44

3,527

Total

164

11,795

To provide the neural network with ground-truth data for learning, it is necessary to manually identify the locations and regions of all aggregate particles present in each stockpile aggregate image. This manual segmentation process is called "labeling," or "annotation." The VGG Image Annotator (VIA) (Dutta and Zisserman 2019) is selected as the tool to ease the labor-intensive manual labeling process. Each aggregate region is marked by a polygon with all vertex coordinates recorded in pixel dimension. These regions are given a label named "rock" so that when processing this image from the dataset, the neural network will search for this label and locate every aggregate region in the image.

The main idea is to label as many particles as possible in a stockpile image according to the following criteria: (a) the polygonal line should carefully approximate the particle boundary with no large deviation from the real shape; (b) one should try to label all human-identifiable particles, except very tiny ones that the naked eye cannot clearly recognize and those that are indistinguishable in dark areas; and (c) incomplete particles at the image boundary should also be labeled so that the segmentation model can show consistent performance at different locations in an image. Example raw and labeled images are illustrated in Figure 5.2. In this example, a total of 213 aggregate particles were manually labeled in the stockpile image.

Following the above procedure and criteria established, 164 stockpile images containing 11,795 labeled aggregate particles constituted the stockpile image dataset for training. This labeled dataset serves as the human vision ground truth for the deep-learning framework described in detail in the next section. 


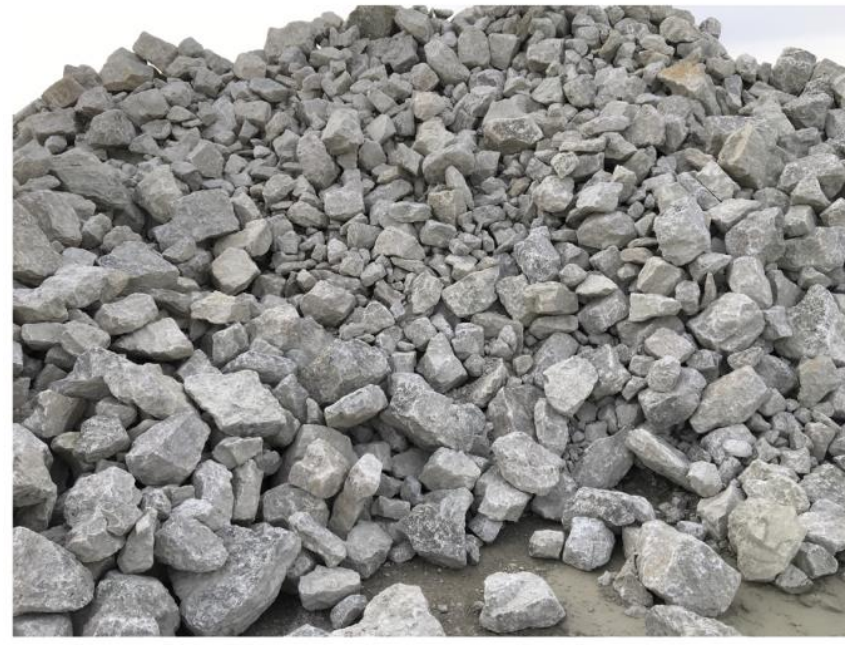

(a)

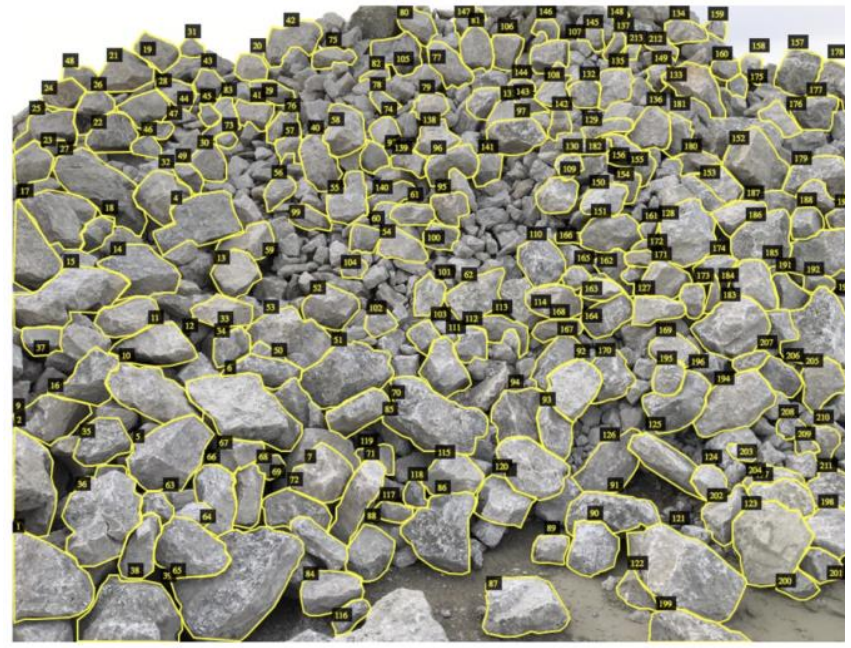

(b)

Figure 5.2. Stockpile aggregate image (a) before labeling and (b) after labeling.

\subsection{DEEP-LEARNING FRAMEWORK FOR AUTOMATED IMAGE SEGMENTATION}

Automated segmentation of stockpile aggregate images aims to identify and extract each aggregate particle, which is essentially an "instance segmentation" problem in the research domain of computer vision. Instance segmentation refers to the general task of detecting and delineating each object of interest appearing in an image, which is a popular area of interest in computer vision (Zhao et al. 2019; Romera-Paredes and Torr 2016). Research developments targeting algorithms related to this task have been applied in many real-life scenarios, such as urban surveillance, autonomous driving, and scene reconstruction.

In the context of a single stockpile aggregate image, each aggregate particle becomes the target instance to be segmented. Inspired by this similarity in concept, this research adopts a recently developed and successful instance segmentation architecture named Mask Region-based Convolutional Neural Network or Mask R-CNN (He et al. 2017), which has proven to be a breakthrough solution for general-purpose instance segmentation tasks. It was first proposed by Facebook AI Research (FAIR) and is recognized as a breakthrough of the instance segmentation task. Mask R-CNN is a flexible and efficient framework for instance-level recognition, which can be applied to other general tasks with minimal modification. For this project's purpose, it was selected for the rock segmentation task. It is an efficient and flexible framework with convenient extensibility for taskspecific applications. The instance segmentation task in Mask R-CNN is divided into an object detection step followed by a semantic segmentation step, and accordingly, Mask R-CNN is composed of two neural networks: Region-based Convolutional Neural Network (R-CNN) for object detection and Fully Convolutional Network (FCN) for semantic segmentation. The model architecture of the neural network is illustrated in Figure 5.3. 


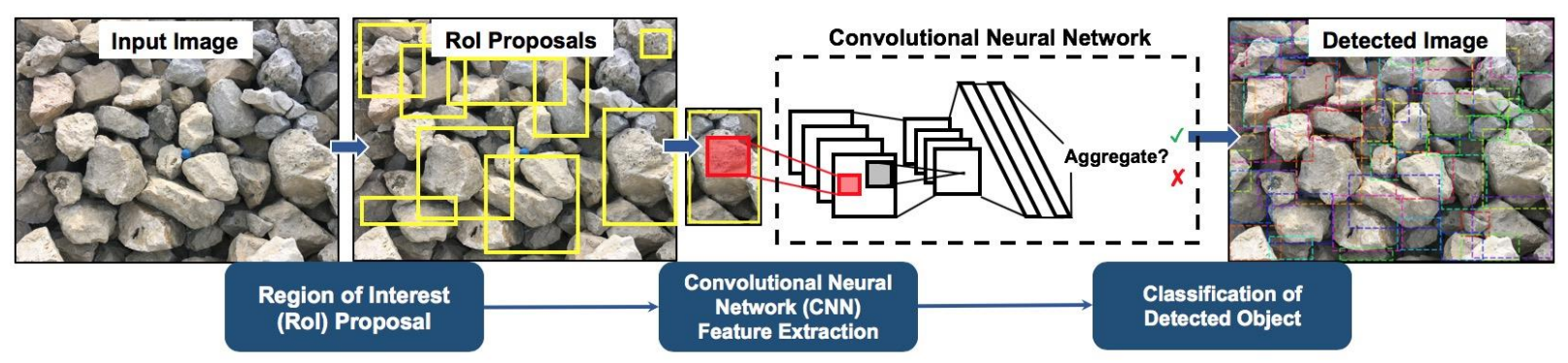

(a)

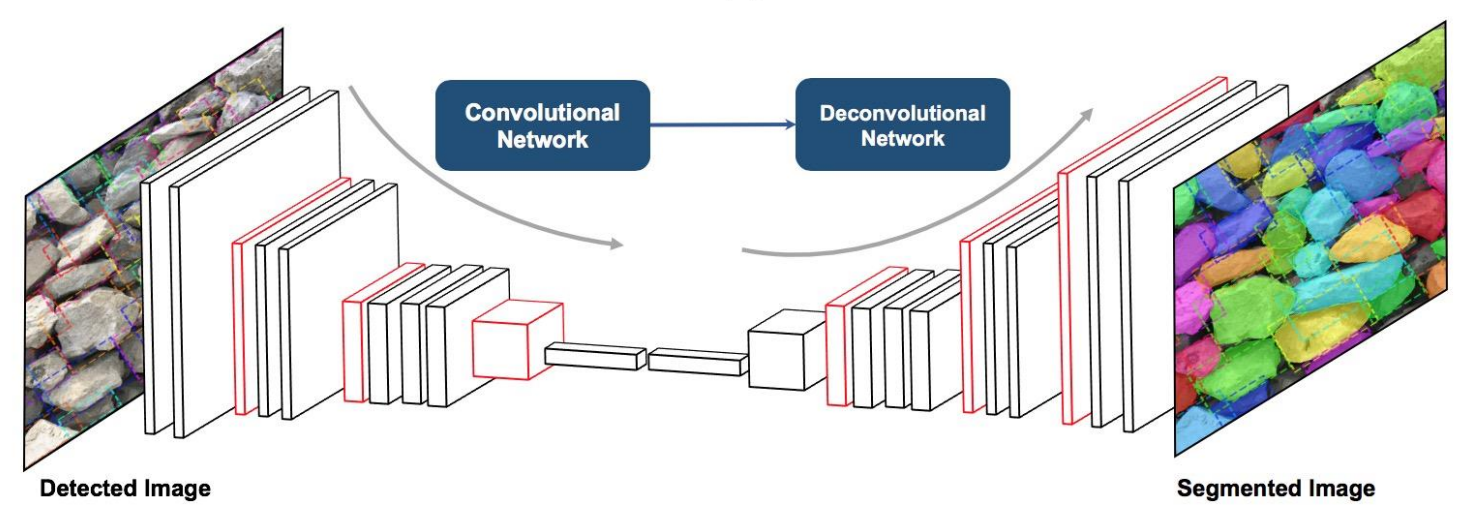

(b)

Figure 5.3. Model architecture of the Mask R-CNN framework composed of (a) Region-based Convolutional Neural Network (R-CNN) and (b) Fully Convolutional Network (FCN).

\subsubsection{R-CNN Framework for Object Detection}

Object detection is employed to estimate the contents and locations of the objects contained in an image. As one of the fundamental problems in computer vision, object detection provides comprehensive information for semantic understanding of the target image. For the stockpile aggregate image segmentation, aggregate particles should be generalized as a target category of the object to be detected. Traditionally, this task involves three stages: region selection, feature extraction, and object classification (Zhao et al. 2019). Following this pipeline, this research adopts the R-CNN architecture that consists of a region proposal scheme and an object classification scheme.

The region proposal scheme simulates the attentional mechanism of the human brain during the object recognition process. The model first generates a large set of Regions of Interest (Rol), or region proposals, using a Region Proposal Network (RPN). The yellow boxes in Figure 5.3(a) are several example region proposals generated during this step. Each region proposal is then condensed into a feature map via the traditional CNN-based feature extraction network. As the next step, the object classification model feeds the feature map into a linear Support Vector Machine (SVM) and reports the object classification and confidence level of each region using non-maximum suppression. At locations with a high confidence level, overlapping bounding boxes are merged into one final bounding box marked as a detected object (He et al. 2017). As a result, R-CNN can efficiently extract high-level features and significantly improve the quality and accuracy of the detected objects. In a general stockpile image, the algorithm is expected to detect, recognize, and locate only valid aggregate particles and distinguish them from other elements such as sky, ground, workers, etc. 
Detected aggregates are marked with colored bounding boxes associated with confidence levels, as illustrated in the detected image in Figure 5.3(a).

\subsubsection{FCN Framework for Semantic Segmentation}

After object detection, semantic segmentation is needed to further extract the valid aggregate pixels inside each bounding box to obtain the particle shape and boundary. During the past few years, significant research effort has been made to accomplish this task accurately and rapidly and has achieved substantial progress. FCN is one of the most powerful models for semantic segmentation; it associates each pixel with an object class description (Long et al. 2015; Arnab and Torr 2017). Fully convolutional, as shown by its name, is a network with pure convolutional and pooling layers, and thereby requires fewer hyper-parameters while preserving high accuracy. The network is composed of a convolutional network followed by a symmetric deconvolutional network. Through the forward inference and backward propagation mechanism, the trained network can take an input image of any arbitrary size and output localized object regions for the designated class. At the pixel level, the network will screen out the invalid non-aggregate pixels and extract the aggregate surface inside the detected bounding box. This semantic segmentation process is illustrated in Figure 5.3(b).

\subsubsection{Training of Neural Network}

The proposed neural network in Figure 5.3 was trained based on the pretrained COCO model using the labeled stockpile image dataset. After the training, a Mask R-CNN model, referred to as the segmentation kernel in the following context, was established for the stockpile image analysis. Following the machine learning concepts, the training of a neural network follows a forward-pass and back-propagation scheme. The forward pass will feed input image(s) to the neural network, and output is generated in the form of segmented image(s). However, because the neural network parameters are randomly initialized at the beginning, these segmentation results can deviate significantly from the ground-truth labeling. This deviation between output and ground truth is calculated by a loss function that quantifies the error. Accordingly, in the back-propagation step, the model parameters of the neural network will update based on the forward pass error. Therefore, the neural network obtains the ability to self-adjust, or "learn," to tackle the segmentation task. The forward pass and back-propagation steps are conducted as an iterative learning process. Note that because of the complexity of the deep-learning framework and the large training dataset, the training process requires a significant allocation of computing power. The training was completed on a desktop workstation with an NVIDIA TITAN Xp Graphical Processing Unit (GPU), 12GB VRAM, and the training process took approximately three days.

The loss of different components of the deep-learning framework is plotted in Figure 5.4 for a total of 50 training epochs. Note that the loss is a unitless metric for the error, and an epoch refers to a complete forward-backward iteration on all the labeled images in the dataset. As seen, each component of the deep-learning framework demonstrates a similar trend of loss decreasing during the training process, indicating that the neural network is learning and improving its capability to complete the segmentation task. Specifically, Figure 5.4(b-d) indicates the neural network has learned to complete the object localization and classification tasks (R-CNN) and the semantic segmentation task (FCN) respectively. Figure 5.4(a) represents the overall learning process of the entire Mask R-CNN network. 


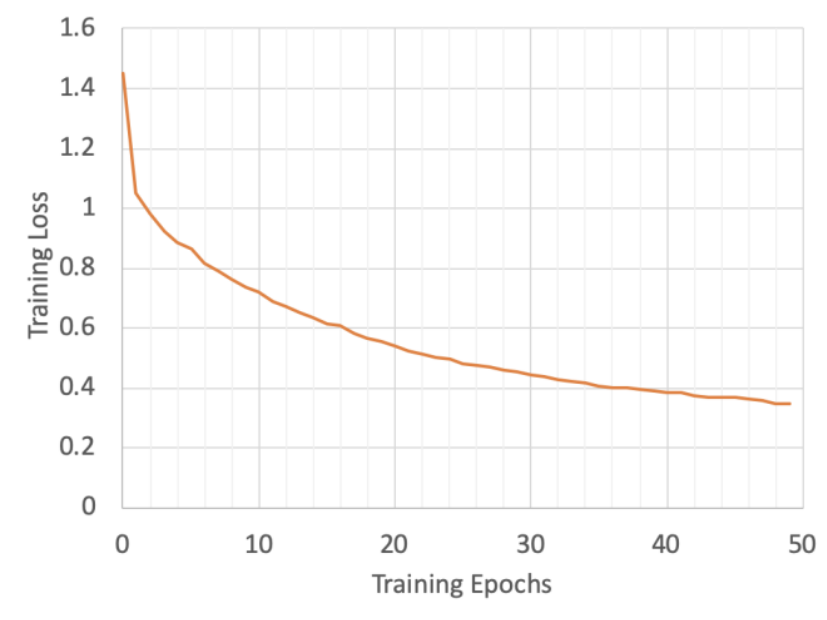

(a)

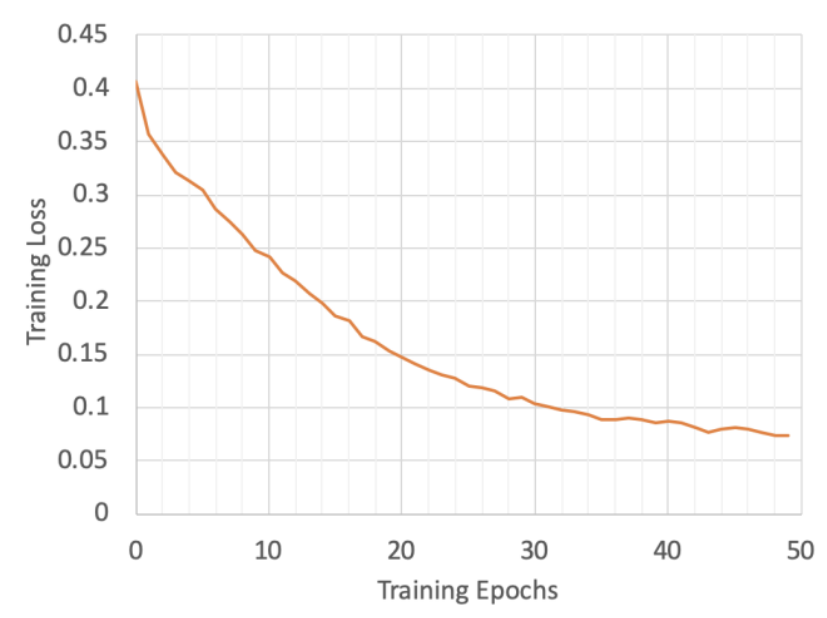

(c)

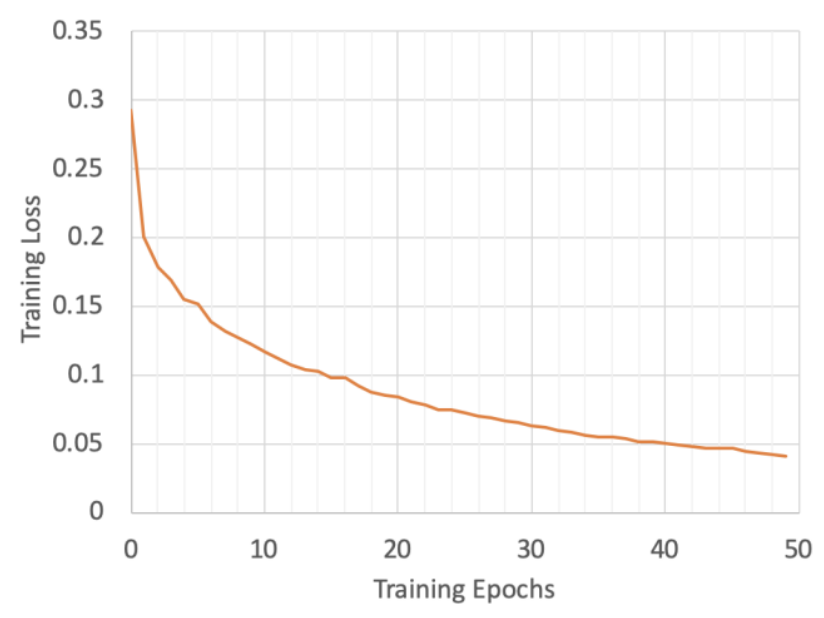

(b)

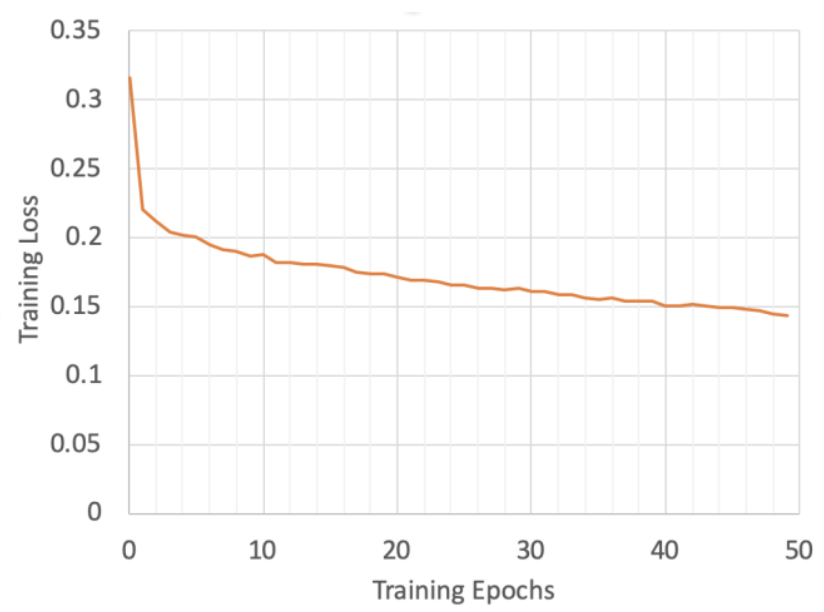

(d)

Figure 5.4. Training loss of (a) entire Mask R-CNN network, (b) R-CNN object localization network, (c) R-CNN object classification network, and (d) FCN semantic segmentation network.

\subsection{MORPHOLOGICAL ANALYSIS ALGORITHMS OF STOCKPILE PARTICLES}

After the successful segmentation, each region that belongs to a different aggregate particle is then fed into the morphological analysis module. The equivalent sizes and Flat and Elongated Ratios (FER) are calculated for these segmented particles and are presented as histogram and cumulative distribution. The unit of length used in the morphological analyses is determined with reference to a 2.25 -in. $(5.7-\mathrm{cm})$ blue calibration ball in the stockpile image.

The equivalent size of a particle used in this research study follows the definition of the Equivalent Spherical Diameter (ESD), which is commonly used to characterize the size of an irregularly shaped object as follows: 


$$
E S D=2 \sqrt{\frac{A}{\pi}}
$$

where $A$ is the measured area of the irregularly shaped object. Users can use other size metrics such as longest, shortest, or intermediate dimension at their discretion.

For the FER calculation, Feret dimensions are used to measure the particle shape along specified directions. Generally, the Feret dimension, also called the caliper diameter, is defined as the distance between two parallel planes restricting the particle perpendicular to the direction of the planes. The calculation of FER needs to find a maximum and a minimum Feret dimension. The maximum, or longest Feret dimension, $L_{\text {max }}$, is first determined by searching for the longest intercept with the particle region in all possible directions. Next, by searching the intercepts along the orthogonal directions against the $L_{\max }$, the minimum or shortest Feret dimension, $L_{\text {min }}$, is obtained. The FER is then defined as the ratio between maximum and minimum dimensions:

$$
F E R=\frac{L_{\max }}{L_{\min }}
$$

Note that an individual particle shape is typically characterized using three morphological factors at three different scales. These are global form (large scale), related to the flatness and elongation or sphericity of a particle; angularity (intermediate scale), linked to crushed faces, corners, and edges of a particle; and, finally, surface texture (small scale), related to the smoothness and roughness of aggregate particles. Other previously developed 2D shape descriptors, i.e., Angularity Index (AI) and Surface Texture Index (STI), quantify the shapes at each scale that are widely considered to be accurate indices in the construction aggregate community. Although characterization of all shape indices is important, this research study limits the focus on the global form using FER, not on the surface texture characterization. This is because (a) such small-scale characterization typically requires an ultra-high resolution that may not be practical; (b) surface texture has been often mechanically characterized in terms of surface roughness (i.e., effect of surface texture) such as friction coefficient (or interparticle friction angle); and (c) surface texture is closely linked to mineralogy and crushed faces.

\subsection{RESULTS AND DISCUSSION}

To validate and visualize the performance of the segmentation kernel, 20 labeled images were randomly selected as the validation set. The validation set typically serves as a benchmark for measuring the performance of trained models, because the images in this set have never been used in the training process. Model performance on the validation set indicates the generality and robustness of the model when processing unseen images.

\subsubsection{Comparison of Image-Segmentation Results}

The kernel takes the images in the validation set as an input and outputs the segmentation results with each aggregate particle marked by a colored mask. Figure 5.5 illustrates the segmentation results on various types of sample images in the validation set as well as comparison with traditional watershed segmentation results. As shown in Figure 5.5(g-i), the segmentation kernel successfully 
completes the image-segmentation task and achieves robust performance on different types of aggregate images, such as separated particles, non-overlapping particles, and densely stacked particles. An interesting phenomenon to note is that although the training dataset contains only stockpile aggregate images, the trained model has gained a more general and consistent skill of segmenting aggregates in different types of backgrounds. This indicates that this segmentation kernel may have learned certain intrinsic morphological features of aggregate particles, and thereby has the potential to process general aggregate images other than the stockpile form.

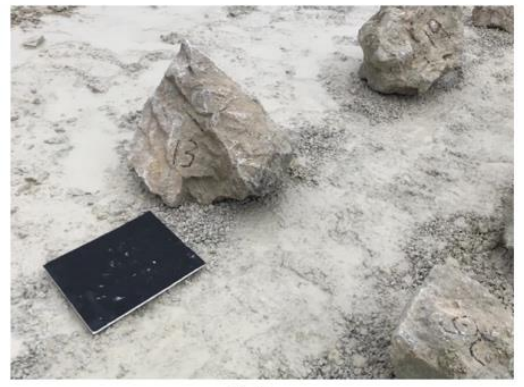

(a)

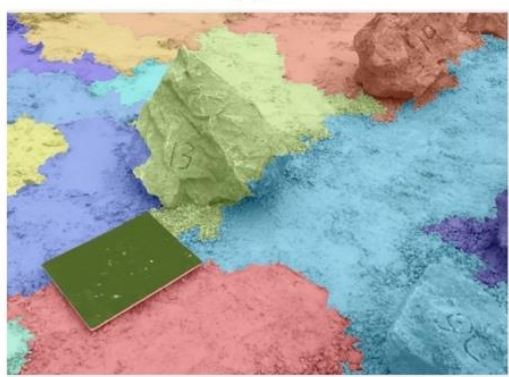

(d)

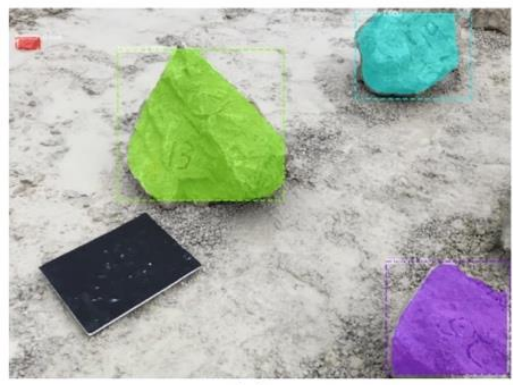

(g)

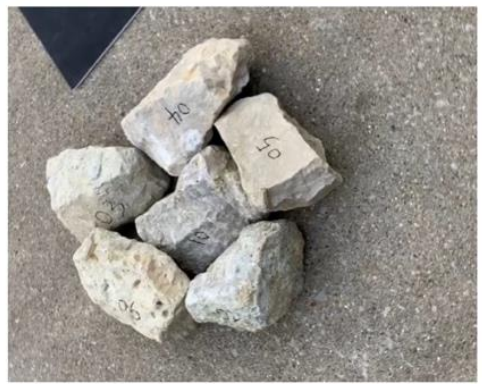

(b)

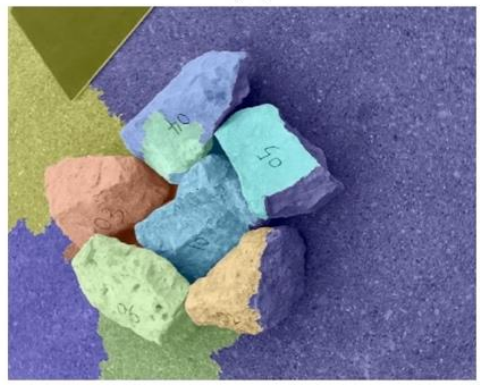

(e)

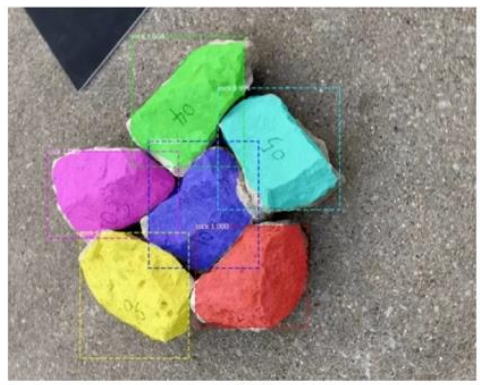

(h)

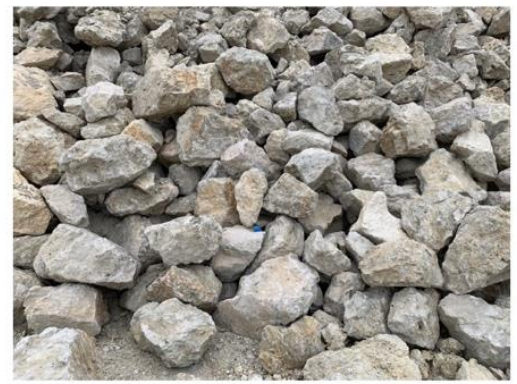

(c)

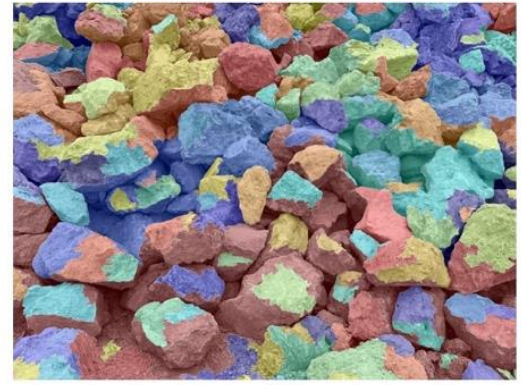

(f)

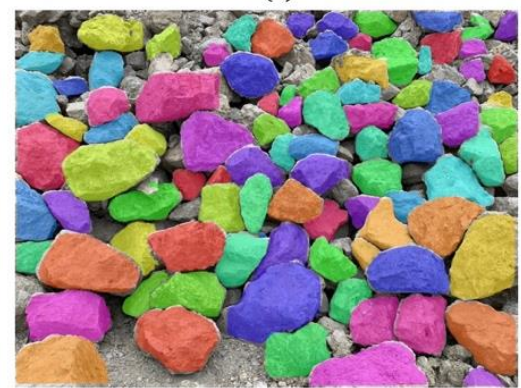

(i)

Figure 5.5. Raw images of (a) separated particles, (b) contacting or overlapping particles, and (c) densely stacked particles. Watershed segmented images of (d) separated particles, (e) contacting or overlapping particles, and (f) densely stacked particles. Mask R-CNN segmented images of (g) separated particles, (h) contacting or overlapping particles, and (i) densely stacked particles.

When compared with the traditional watershed segmentation results, the Mask R-CNN segmentation results have much better partitioning along the aggregate boundary (see Figure 5.5). This can be explained by the different mechanisms behind the watershed and CNN-based methods. The watershed method tries to separate all pixels in the image based on the regional intensity with no clues on the semantic meaning of an object's existence. The CNN-based method, on the other hand, 
first conducts object detection and locates all potential particle regions and then segments the aggregate pixels in detail. This mechanism, along with the confidence value reported for each detected region, ensures that the model would rarely recognize irrelevant pixels as aggregates. Additionally, without the object detection mechanism, the watershed algorithm tends to categorize every pixel in the entire image into one of the regions, which is counterintuitive in the context of aggregate image segmentation. For example, in Figure 5.5(d-f), watershed segmentation results include many non-aggregate fragments such as the ground or blackboard, which are very difficult to eliminate by fine-tuning the algorithm parameters. Additional post-processing steps may be needed to select the valid aggregate regions before conducting the morphological analyses. Conversely, CNNbased segmentation identifies the greatest number of individual particles in visually reasonable shapes and thus requires little or no post-processing, as illustrated in Figure 5.5(g-i).

Another important observation is that the problematic shadow issue is well handled in the CNNbased segmentation results. The shadow effect has always been a challenge during digital image processing, because computer vision algorithms have difficulty distinguishing between an on-surface shadow and a cast shadow, especially when the algorithms rely heavily on human-defined features. In Figure 5.5(d-f), the watershed method is also misled by the shadow such that several aggregate particles are segmented into two adjacent regions along the light-shadow divide. The Mask-RNNbased method - which better emulates the perception of the human vision system-unambiguously extracts the whole particle. This is because the convolutional scheme of this neural network recognizes implicit features among multiple levels of abstraction instead of focusing on local features such as texture or pixel intensity. Such advantage enhances the reliability and precision of each segmented aggregate particle as compared to the watershed method, as clearly illustrated in Figure 5.5(e) and Figure 5.5(h).

Additional selected segmentation results are presented in Figure 5.6. Note that in Figure 5.5(i) and Figure 5.6, not all human-identifiable particles are detected and segmented. These non-segmented regions generally include two types. First, particles that are highly occluded are not detected, because unrecognizable, highly incomplete, or extremely tiny particles were not labelled during the manual labeling process. They are deliberately screened off because such particles may become outliers during the morphological analysis as they affect the accuracy of the total particle statistics. The trained model follows this feature of the labeled dataset and is therefore selective as well. On the other hand, there are valid aggregate particles that are not detected by the segmentation kernel. They are usually particles with special shape, orientation, color, or texture that are quite different from labeled ones in the dataset. This indicates that the dataset should be further enlarged to account for robustness. 
Raw Stockpile Image
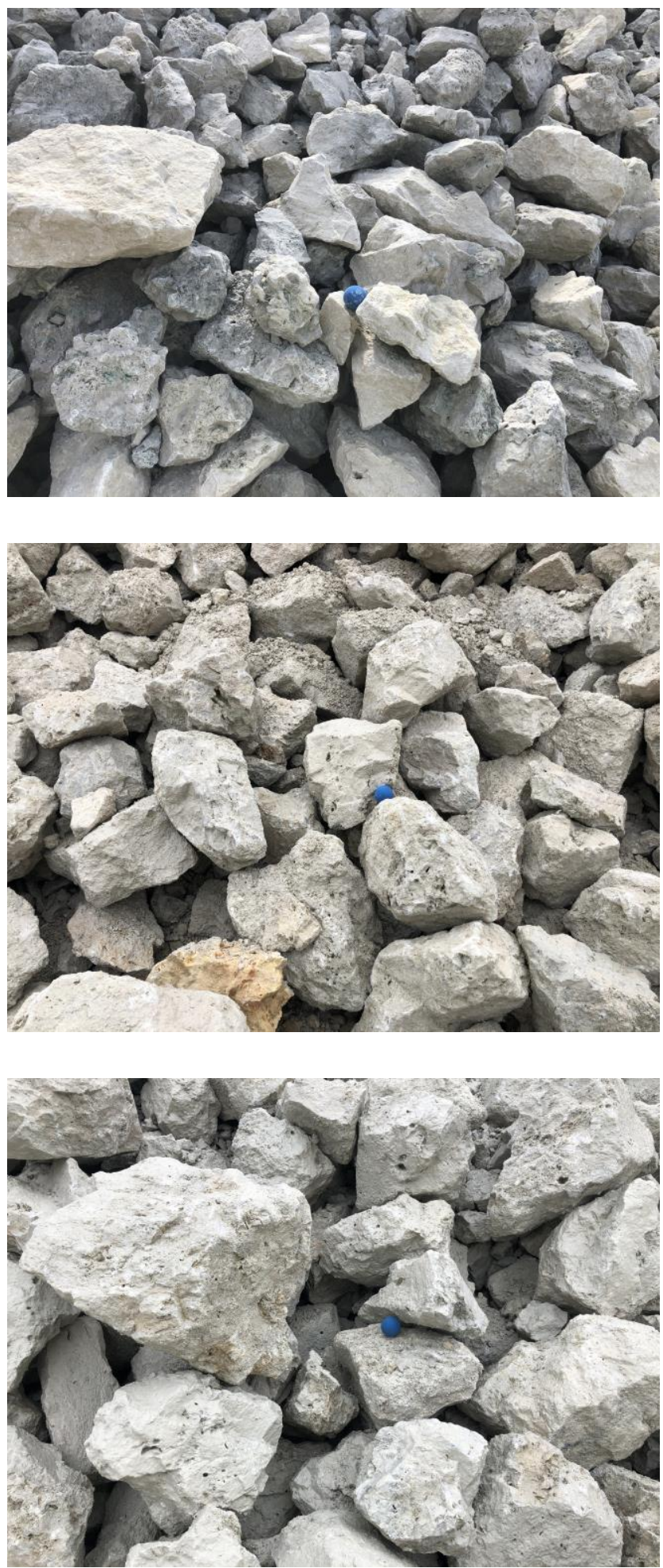

Segmented Image
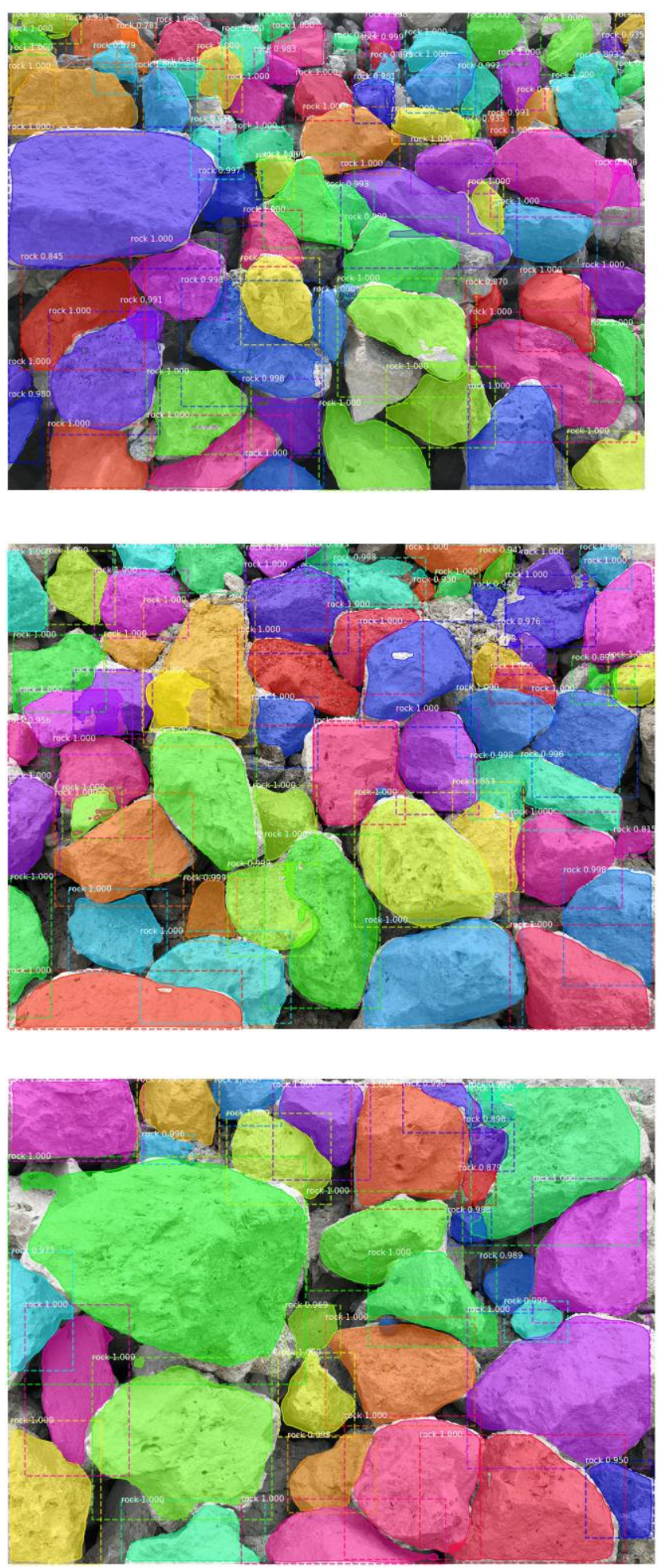

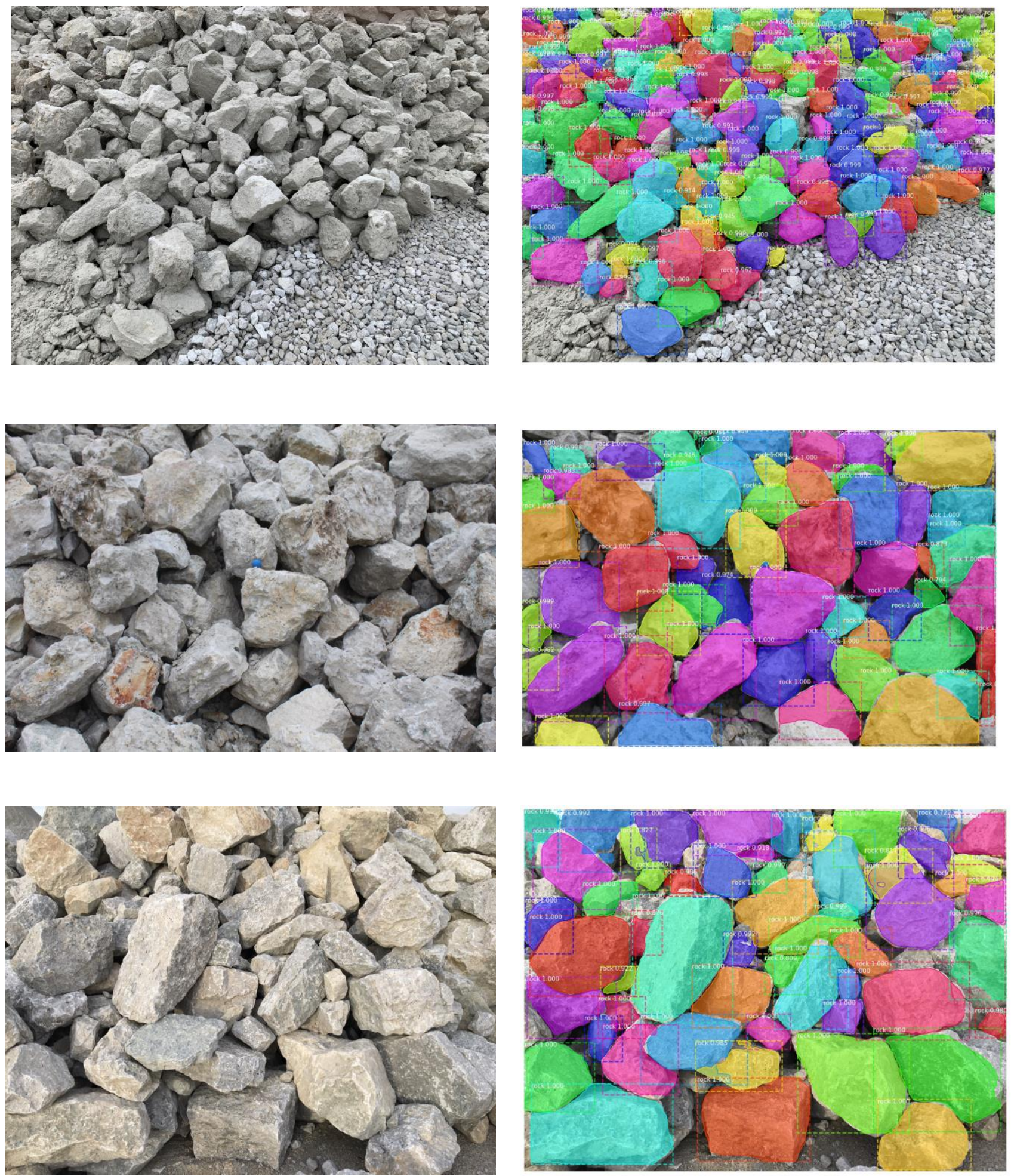

Figure 5.6. Additional stockpile image-segmentation results. 


\subsubsection{Morphological Analysis Results}

After the successful segmentation, each region that belongs to a different aggregate particle is then fed into the morphological analysis module. The equivalent sizes and FERs are calculated for these segmented particles and are presented as histogram and cumulative distribution. The size and shape metrics are also calculated for the corresponding labeled image and are plotted as the ground-truth comparison. The morphological analysis results for an example stockpile image in the validation set (shown in Figure 5.5[i]) are presented in Figure 5.7. The unit of length in the following analyses is determined with reference to a 2.25 -in. $(5.7-\mathrm{cm})$ blue calibration ball in the image.

For the example stockpile image in Figure 5.7(a), 93 particles are segmented by the imagesegmentation kernel, and a total of 100 particles are identified during the ground-truth labeling process. From the particle-size analysis results in Figure $5.7(b-c)$, the sizes of the aggregate particles are between 2 in. and 13 in., with about 70\% of the particle sizes ranging from 3 in. to 8 in. The segmentation results demonstrate good agreement with the ground truth in histogram counts and cumulative distribution. From the FER analysis results in Figure 5.7(d-e), the FERs range from 1.0 to 3.0, and more than $90 \%$ of the particles have FERs less than 2.0 . The segmentation results again capture the trends in the ground-truth histogram and cumulative distribution. Both analyses show reasonable statistical distributions for the morphological properties in an aggregate stockpile and achieve good agreement with the ground-truth labeling. The particle-size distribution curve indicates a uniform gradation of the stockpile. The FER distribution, influenced by the crushing process for this batch of aggregates, implies that more cubical particles were produced instead of long and slender ones.

Note that the morphological analysis presented herein is an example analysis with simplified analytical components. Users should be attentive to the following aspects during a formal and comprehensive morphological analysis step. First, it is highly recommended for users to take images from a perpendicular direction against the stockpile slope. The images in the training dataset have no restrictions on viewing angle, because they are meant for the development of the segmentation kernel. But the images for morphological analysis should be normal facing in order to minimize the perspective distortion (or foreshortening) effect of images. Images taken in this way can ensure the accuracy and reliability of the morphological analysis results. In addition, incomplete particles segmented at the image boundary may be removed from the morphological analysis because such shapes are caused by artifacts at the image boundary. Secondly, advanced morphological analysis modules - such as the ones in existing aggregate imaging systems - can be used for a more comprehensive characterization of particle shape regarding the form, angularity, and texture. Finally, and as a limitation, because only the stockpile surface is visible to the users, note that the morphological analysis results only represent the aggregate statistics for the surface particles in a stockpile. 


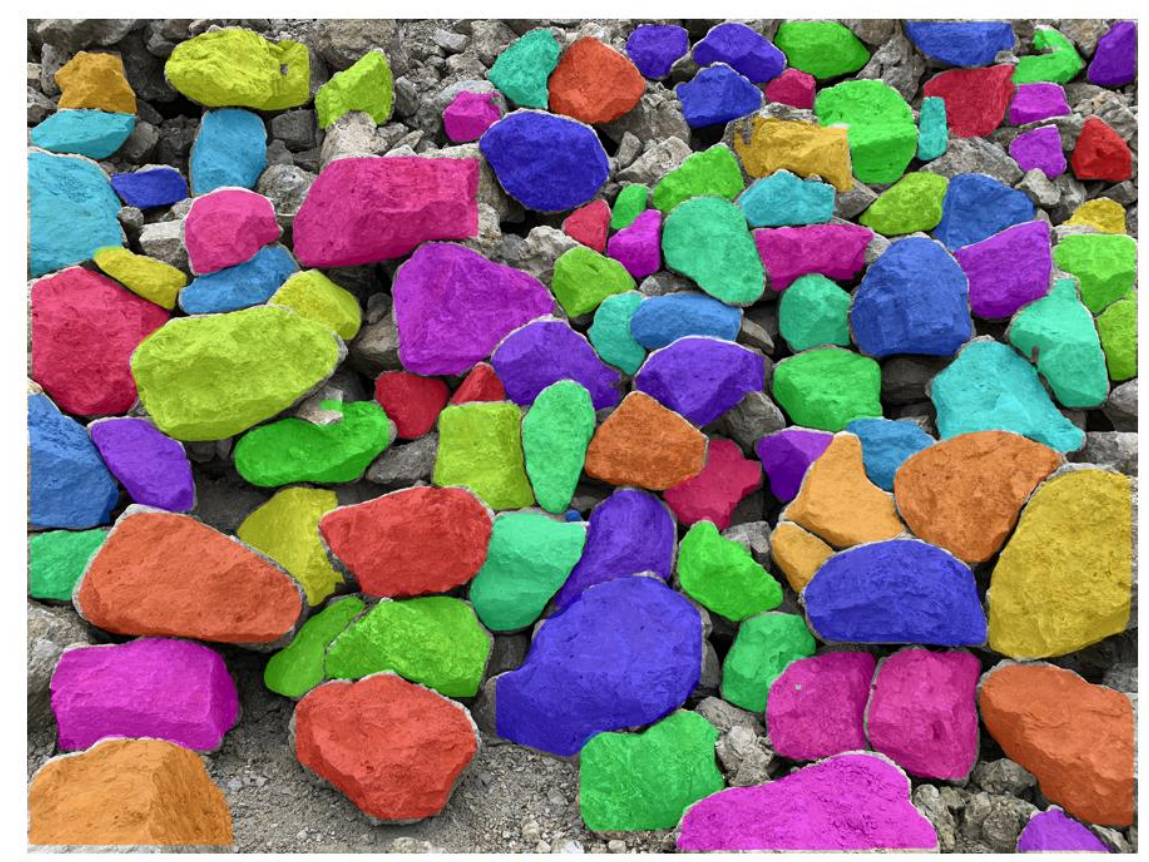

(a)

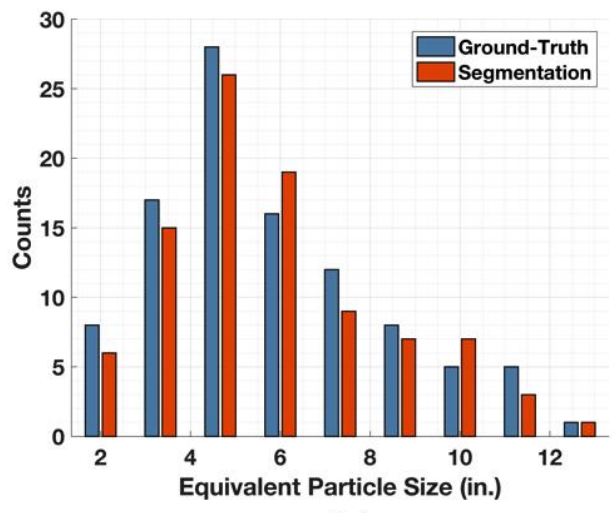

(b)

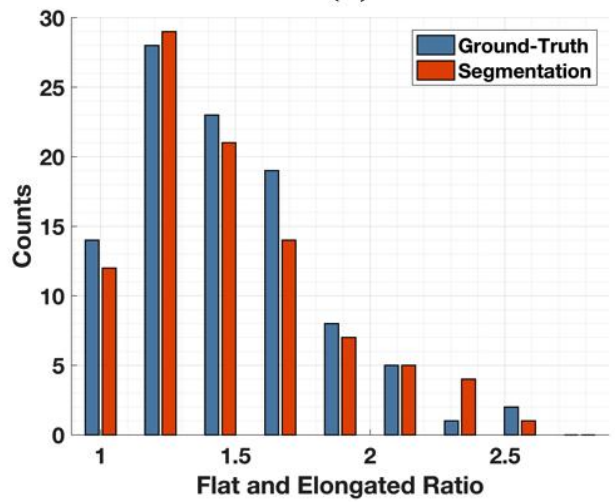

(d)

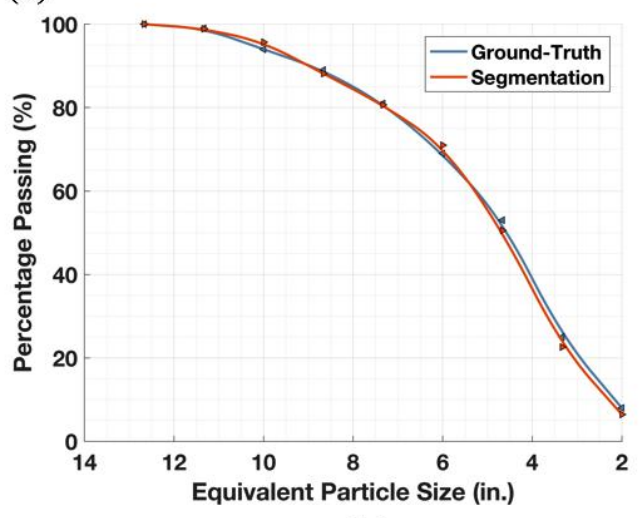

(c)

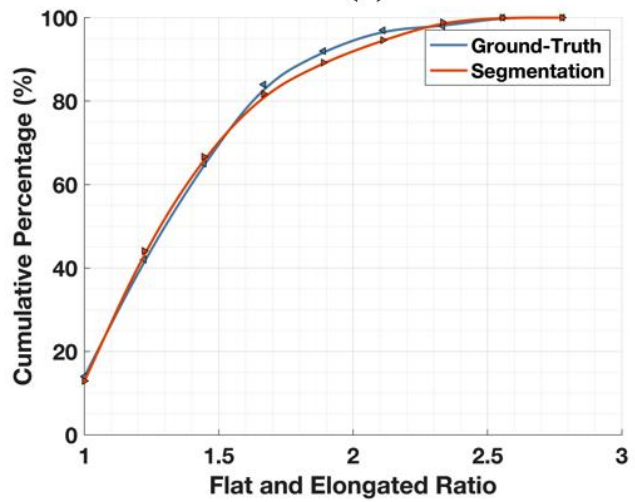

(e)

Figure 5.7. (a) Mask R-CNN segmented image (enlarged from Figure 4[i]); (b) histogram distribution and (c) cumulative distribution curves for equivalent particle size; and (d) histogram distribution and (e) cumulative distribution curves for flat and elongated ratio. 


\subsubsection{Statistical Analyses of Segmentation Results}

To evaluate the performance of the segmentation results, two important statistical indices, completeness and precision, are selected as the performance indicators. They are widely used for model evaluation in image-segmentation problems. The illustration of these two metrics is given in Figure 5.8. To assess the completeness of the segmentation results, the ratio between the number of segmented particles and the number of ground-truth labeled particles is calculated. This defined ratio describes the percentage of particle regions correctly detected as compared to the ground-truth labeling, which measures the overall performance of the object detection step. As for the precision metric, the Intersection over Union (IoU) score calculates the percent overlap between the segmented particle mask and the corresponding ground-truth mask. This metric measures the number of pixels in common between the segmented and ground-truth masks divided by the total number of pixels present across both masks, as given in Equation (5.3).

$$
\text { IoU }(\%)=\frac{\text { Segmented } \cap \text { Ground }- \text { Truth }}{\text { Segmented } \cup \text { Ground }- \text { Truth }}
$$

where "Segmented" denotes the region of segmented mask and "Ground-Truth" denotes the groundtruth labeled mask. The average loU score of all segmented particles in an image measures the overall accuracy of the semantic segmentation step.

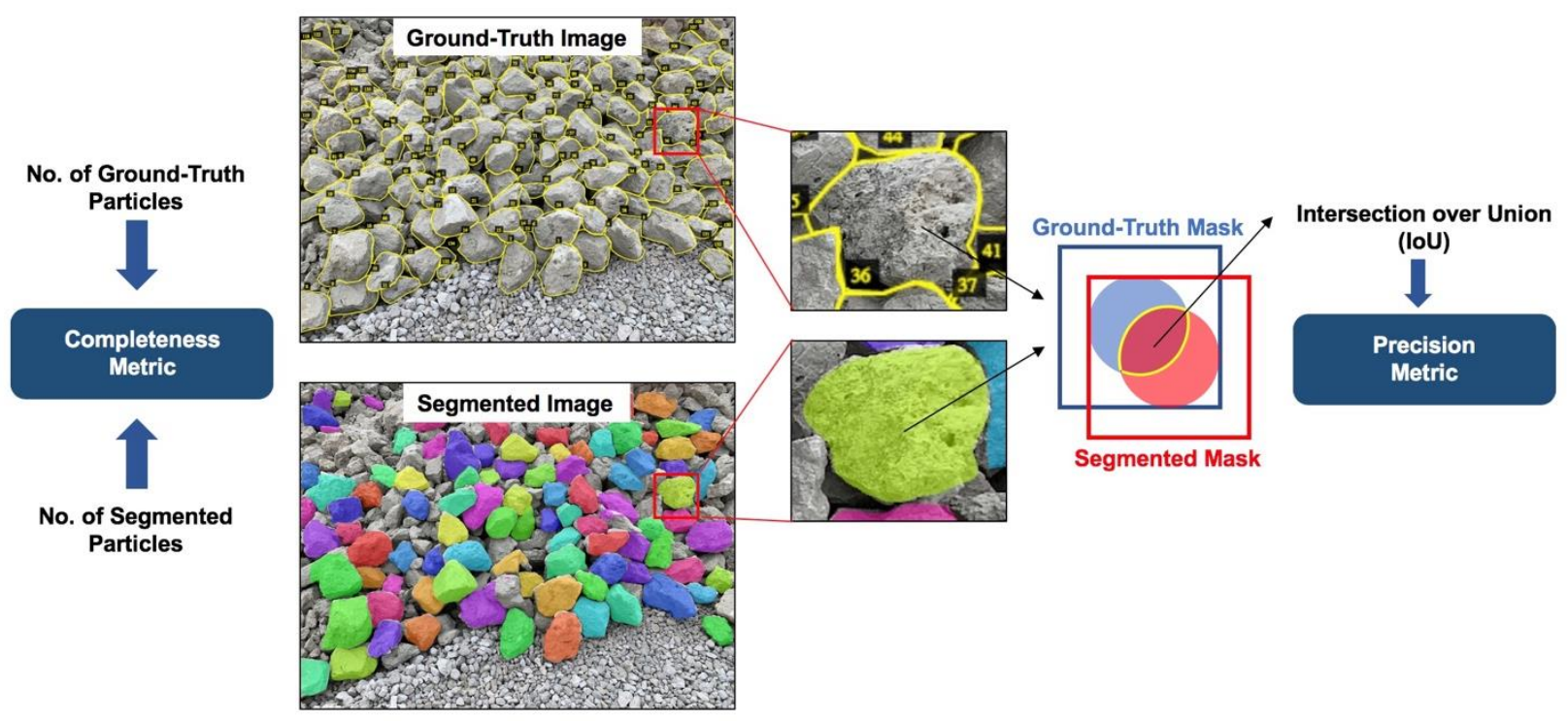

Figure 5.8. Completeness and precision metrics used to compare the segmentation results with the ground-truth labeling.

Following the completeness and precision metrics, the model performance is evaluated on 15 stockpile images from the validation set. Note that there were in total 20 riprap images in the validation set, including five non-stockpile images of separated or non-overlapping particles (see Figure 5.5[a-b]). To better measure the model performance on stockpile images, those five images were excluded from the completeness and precision validation and only stockpile images were selected. As listed in Table 5.2, the average completeness and precision values are $88.0 \%$ and $86.7 \%$, 
respectively, which are both considerably high for dense image-segmentation and analysis tasks. They indicate that the model has been well trained to detect and segment only those "true" aggregate regions instead of reporting ambiguous aggregates with a large error, which is ideal for stockpile image segmentation.

Table 5.2. Completeness and Precision Results of Randomly Selected Validation Set Images

\begin{tabular}{ccccc}
\hline ID & $\begin{array}{c}\text { Number of } \\
\text { Labeled Particles }\end{array}$ & $\begin{array}{c}\text { Number of } \\
\text { Segmented } \\
\text { Particles }\end{array}$ & Completeness & Precision \\
$(-)$ & $(-)$ & $(-)$ & $(\%)$ & $(\%)$ \\
\hline 1 & 70 & 67 & 95.7 & 87.7 \\
\hline 2 & 56 & 53 & 94.6 & 88.3 \\
\hline 3 & 131 & 102 & 77.9 & 87.4 \\
\hline 4 & 73 & 65 & 89.0 & 86.1 \\
\hline 5 & 111 & 92 & 82.9 & 83.2 \\
\hline 6 & 99 & 84 & 84.8 & 86.9 \\
\hline 7 & 114 & 91 & 79.8 & 83.7 \\
\hline 8 & 106 & 88 & 83.0 & 87.9 \\
\hline 9 & 115 & 96 & 83.5 & 86.8 \\
\hline 10 & 117 & 95 & 81.2 & 87.5 \\
\hline 11 & 60 & 56 & 93.3 & 88.0 \\
\hline 12 & 149 & 127 & 85.2 & 87.5 \\
\hline 13 & 56 & 51 & 91.1 & 87.2 \\
\hline 14 & 62 & 62 & 100.0 & 87.8 \\
\hline 15 & 116 & 114 & 98.3 & 85.3 \\
\hline Average & & & 88.0 & 86.7 \\
\hline Standard Deviation & & & 1.5 \\
\hline
\end{tabular}

The average completeness value shows that more than $85 \%$ of aggregate particles can be identified as compared to the ground truth, and those particles can be segmented with over $85 \%$ accuracy. The model misses about $10 \%$ to $20 \%$ of the ground-truth labeled particles, which can be explained by its conservative behavior during the detection step. By setting the confidence threshold at 0.7 , the model only reports the aggregates with a relatively high precision. This often leads to a lower completeness rate, because non-aggregate regions are screened off. For morphological analysis, the target is to process reliable aggregate regions rather than poorly segmented ones. Hence, it would be adequate to maintain this conservative behavior and further improve the completeness of model performance by retraining the model with a more comprehensive dataset. In addition, the standard 
deviation values for completeness and precision are $7.1 \%$ and $1.5 \%$, respectively. This implies good generality and robustness of the model performance on different unseen input images.

Furthermore, as compared to existing aggregate imaging systems, the trade-off between the number of analyzed particles and the precision of segmented particle shape is noteworthy. These aggregate imaging systems are not efficient for massive evaluation because of the additional setup and human effort required to separate aggregate particles. But, the characterized shapes are of high precision, because the particles are all analyzed separately under controlled conditions. Namely, the morphological analyses in those systems provides high-precision analysis on a small sample portion of aggregates.

In contrast, this research study has developed an efficient massive analysis based on stockpile images, but the particle shapes are of medium precision because of the inevitable occlusion and overlapping effect occurring in stockpile aggregate images. This intrinsic difference is recognized, and this approach has great practical merits. The reasons are twofold. First, this new approach may better serve the tasks when quick and massive analyses of aggregate stockpiles are demanded, e.g., in a quarry or a construction site, especially during the time-sensitive quality control process. Second, the stockpile image analysis does not require additional setup and can handle in-place evaluation of small- to large-sized aggregates, while the existing systems are limited to small-sized aggregates under laboratory conditions. In addition, as for realistic representation of an entire stockpile of aggregate material, more statistical analysis is needed to determine whether a high-precision result from a small sample group or medium-precision result of the whole stockpile surface will be more representative and informative.

\subsection{SUMMARY}

This chapter presented an innovative approach for automated segmentation and morphological analyses of stockpile aggregate images based on deep-learning techniques. A task-specific stockpile aggregate image dataset was established from images collected from quarries in Illinois. Individual particles from the stockpile images were manually labeled on each image associated with particle locations and regions. A state-of-the-art object detection and segmentation framework called Mask R-CNN was then implemented to train the image-segmentation kernel, which enables userindependent segmentation of stockpile aggregate images. The segmentation results showed good agreement with ground-truth labeling and improved the efficiency of size and morphological analyses conducted on densely stacked and overlapping particle images. Based on the presented approach, stockpile aggregate image analysis can become an efficient and innovative application for field-scale and in-place evaluations of aggregate materials. 


\section{CHAPTER 6: SOFTWARE DEVELOPMENT FOR RIPRAP STOCKPILE CHARACTERIZATION}

\subsection{INTRODUCTION}

This chapter presents the software development details for the riprap stockpile image analysis program. The primary outcome of this research study is a computer vision software to conduct riprap and large-sized aggregate gradation and to shape classification in the field. The deliverables related to the computer software include: (a) a user-independent, automated segmentation algorithm exclusively developed for field imaging of riprap and large-sized aggregates; (b) a size and shape analysis algorithm to characterize riprap particles in stockpiles; (c) a software program with userfriendly Graphical User Interface (GUI) for easy input and output of data; and (d) a user manual including best practices for capturing images and demonstrating how to use the software and interpret the resulting analysis.

\subsection{REVIEW OF SOFTWARE ENGINEERING CONCEPTS}

Software development is a complicated process that requires careful planning and execution to transform the goals into a software system. Developers must react timely and aggressively to meet ever-changing requirements (Rehman and Paul 2003). Maintaining software quality hinders fastpaced software development, as many testing cycles are necessary to ensure quality products (Pusuluri 2006).

\subsubsection{Software Life Cycle}

Software development, in contrast to common thought, is not just "writing code." The life cycle of one software development project comprises many stages, which can be summarized as follows: 1 ) requirement gathering, 2) writing functional specifications, 3) creating architecture and design documents, 4) implementation and coding, 5) testing and assurance, 6) software release, and 7) documentation. All stages/activities are necessary for a project to go through in order to produce a high-quality software system (Rehman and Paul 2003).

Following the predictable path of the software development life cycle, developers first understand and evaluate the problem/goal. Next, they identify the requirements of a solution to the problem. These requirements are studied in-depth to develop the architecture of the solution that will satisfy the requirements. The solution architecture provides the organizational details for designing the individual solutions (Eddie 2007). Once the architecture and detailed solution designs have been finalized, construction/implementation is the next focus. All work should be carefully tested before integration into the software system. Any further modification to the software system will be treated as maintenance, which will start a new iteration of the software life cycle (Eddie 2007).

\subsubsection{Lifecycle Model of Software Development}

A software process is a detailed series of steps during the software development project. To facilitate reasoning about software processes following the software life cycle, it is helpful to create specific 
abstract groupings of software processes, which is commonly known as the lifecycle model (Feiler et al. 1993).

Because the software development for this project does not require a huge amount of coding, the most-used model, code-and-fix, is selected as the desirable model. The code-and-fix model involves little/no overhead, and it is applicable for small projects and short-lived prototypes. The code-and-fix model starts with sufficient requirements and system specification analysis to begin coding. Once the software system works, developers rework and add more code to the system until it meets all requirements of the project.

\subsection{DESIGN AND CONTROL LOGIC OF GRAPHICAL USER INTERFACE (GUI)}

The 2D stockpile aggregate images captured by the field-imaging kit need to be processed to yield a collection of image segments that approximately capture the individual aggregate particles. These output segments are used for determining individual aggregate particle size and imaging-based shape/morphological indices. Further, aggregate size and shape property distributions should be estimated over the entire stockpile image. The visual description of different image-processing steps is depicted in Figure 6.1, and a tentative version of the GUI is illustrated in Figure 6.2. The control logic of the GUI is divided into three panes: user input/output (I/O) panes, result display panes, and a summary pane.

\subsubsection{User I/O Panes}

One of the major differences between a research-oriented algorithm and an industry-oriented software application is the convenient handling of user input and output procedure. To ease this procedure, the input and output module of the software is designed to allow different work modes at the user's discretion.

For aggregate producer QA/QC tasks, the user may have to either process a single riprap stockpile image or, more practically, to process a batch of stockpile images taken for a specific group of riprap material. The input module of the software provides both work modes for the user. The user will enter "single" mode upon selection of a single image file or enter "batch" mode when selecting multiple image files. The diverging control logics of the "single" and "batch" modes are handled on the back end, such as different folder settings, segmentation runtime modes, interactive result illustration, etc. After choosing the input mode, the user will be asked to specify an output folder path as well. For every image selected as input, a corresponding result folder with the same name of the input image will be created automatically under the specified output folder. If the folder exists, its content will be cleared before new results are written. This design adopts a hierarchical way of folder configuration, such that all analysis results of each stockpile image are collected and well organized. 


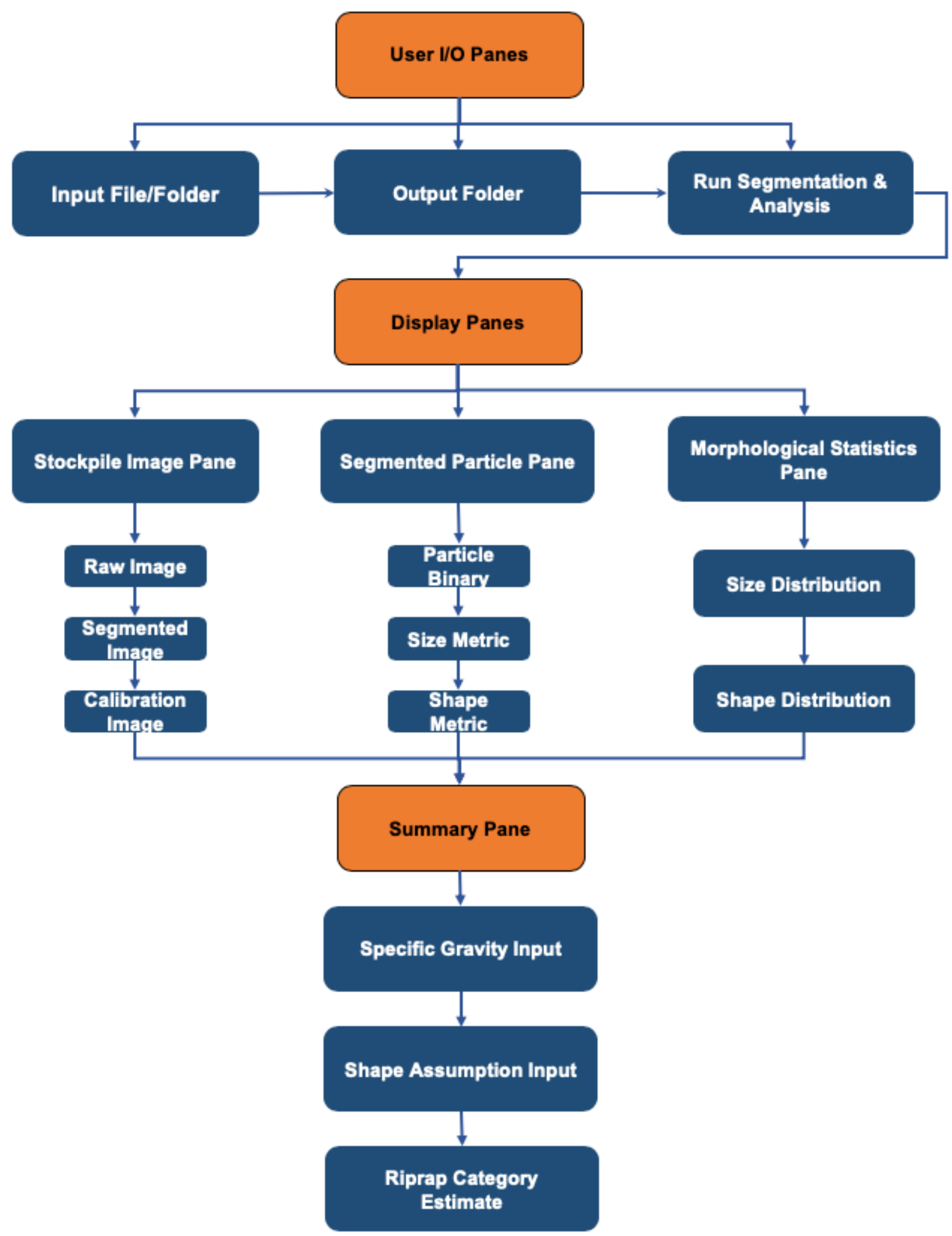

Figure 6.1. Schematic drawing of Graphical User Interface (GUI) control logic. 


\subsubsection{Result Display Panes}

After specifying the input and output folder, the user can run the segmentation and morphological analysis in one click, and all results will be displayed in separate panes for different types of information.

First, the stockpile images at different analysis stages are displayed under the stockpile image pane, as shown in Figure 6.1. The original stockpile image is displayed on the raw image tab of this pane, followed by the segmented image (with an example image shown in Figure 5.7[a]) and the calibration image. Note that the calibration image refers to the image with calibration ball identified, where the calibration data is obtained by a separate module and is used in the following morphological analysis steps. More details about the calibration module can be found in the software user manual. The user can use arrow keys to browse through the input images and get synchronous display as the image is switching.

The stockpile image pane provides an overview of the segmentation results, while the segmented particle pane displays the morphological details of each segmented particle, as shown in Figure 6.1. The binary silhouette image of each segmented particle is shown on this pane, along with particle ID, size metric, and shape metric displayed in the text box. The user can use arrow keys on this pane to scan through segmented particle information associated with the currently active input image in the stockpile image pane.

In the morphological statistics pane, grain size and shape cumulative distribution are obtained from analyzing $2 \mathrm{D}$ stockpile images by using the segmentation techniques described in Chapter 5 . The user can switch between the size and shape distributions by using arrow keys.

\subsubsection{Summary Pane}

The stockpile image pane and the result display pane offer both general and detailed views of the image segmentation and morphological analysis results, but high-level information should be extracted and summarized to provide practical outputs from an engineering perspective. Therefore, the summary pane is designed to allow certain site-specific parameter input in order to enable flexible user interaction with the image analysis data. For example, the user can input a specific gravity value in the conversion from $2 \mathrm{D}$ size to riprap weight; the user can also input a particle shape assumption parameter (ellipsoid shape with certain flat and elongated ratio) to further improve the weight estimation results. Default values will be set based on the single-rock volumetric study to do automatic conversion, if the user chooses not to provide site-specific data. After the site-specific information is input by the user, cumulative percentages at different weight values are calculated to facilitate the size category determination process for QA/QC purposes.

For more details, please refer to the software user manual released along with this project final report regarding the user interface screenshots, step-by-step usage instructions, output file/folder structure, etc. 


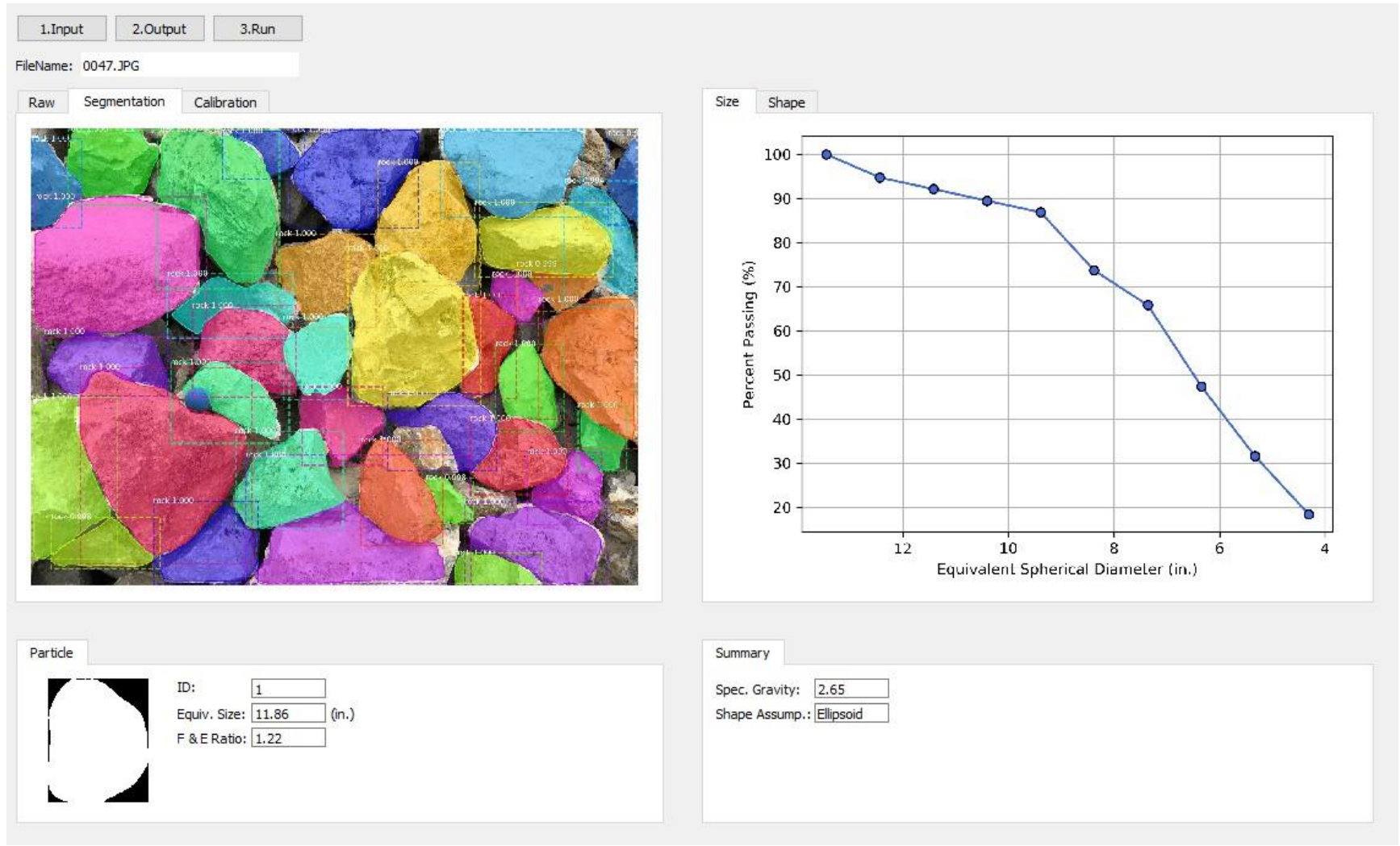

Figure 6.2. Screenshot of graphical user interface.

\subsection{SUMMARY}

This chapter summarized the software development effort towards a practical, user-independent, and user-friendly image-segmentation software targeted in this research study. A software application was developed as the riprap or large-sized aggregate evaluation kit modules for size/shape analyses of individual aggregate particles captured using the field-imaging kit. The research team reviewed and followed professional software engineering guidelines during the developmental stages. Riprap stockpile image analysis algorithms were programmed and integrated into a standalone software application with easy-to-use user interface. The design of the GUI and control logic was intended to provide a fluent workflow for use in industry engineering practice, including system directory setting of input and output file path, synchronous results display and switching, front-end and back-end interaction, user interaction, etc. A user manual will be provided along with this final report for software usage and technical support, and the functionalities of the software will be tested with IDOT engineers. 


\section{CHAPTER 7: CONCLUSIONS AND RECOMMENDATIONS}

\subsection{INTRODUCTION}

Determination of particle-size distribution or gradation of riprap and other large-sized aggregates, e.g., aggregate subgrade, is always a challenge in the field. Individual rocks are often weighed to correlate particle weight to its dimensions, which is a time-consuming and labor-intensive task that is also hard to judge by inspectors. The main benefit of this R27-182 research project is the capability to offer riprap and large-sized aggregate size and shape property testing in the field, at a quarry, or at a construction site, using imaging techniques to determine size/shape properties with improved accuracy and without the need to weigh single particles. This is a priority because IDOT engineers and field inspectors spend considerable time and effort in riprap sizing at approximately 50 sites in Illinois annually.

Implementation of the advanced field-imaging technology that was fully developed in this project will help to accurately determine if riprap material meets gradation specifications. The proposed system, which utilizes stockpile image-segmentation algorithms and the morphological analysis algorithms developed for size and shape indices, is sustainable for field applications. Better property characterization and optimized material selection can be achieved to improve designs through effective quality control, reduced costs, increased life cycle and sustainability with less environmental impacts, and minimum labor and energy consumption. Major cost savings in terms of personnel time, transportation, and laboratory equipment and facility use will be realized because samples need not be collected from the field for detailed laboratory analysis.

The primary objective of this research project was to develop a convenient, nonintrusive fieldevaluation system based on imaging, whereby an engineer or inspector can take multiple photos of the riprap and large-sized aggregates and determine the gradation (size distribution) and shape properties with the help of calibration balls used in image analyses. Imaging systems for both single riprap rock and stockpiles were developed, validated, and integrated into a software application with GUI. Both systems have demonstrated good agreement with ground-truth data and showed future potential as state-of-the-art size and shape characterization techniques for riprap and large-sized aggregates.

\subsection{SUMMARY OF FINDINGS FROM THE SINGLE-PARTICLE STUDY}

The following conclusions can be drawn related to the single-particle field-imaging system for the characterization of size and weight information of individual riprap rocks and large-sized aggregates:

- A comprehensive review of the current practice and literature showed that characterizing the volumetric properties of field riprap in a quantitative manner has not been an area that benefited from the technology advancements in image processing. The state-of-the-practice methods by engineers and practitioners rely on visual inspection and hand measurements.

- A field-imaging system was designed and built as a portable and versatile toolkit for the convenience of efficient and reliable image-acquisition needs. Image segmentation and 3D 
volumetric reconstruction algorithms were developed for single rocks with the capabilities of segmenting object information under uncontrolled field lighting conditions and reconstructing the object three-dimensionally with necessary calibration and correction.

- The robustness and accuracy of the developed algorithms were studied through field-imaging tasks at two aggregate production sites. Good agreements between the ground-truth volume/weight measurements and the image-based volumetric reconstruction results were achieved.

- Comparisons were also made between the image-based volumetric reconstruction results and the state-of-the-practice hand measurements. Significant improvements could be achieved using the proposed field-imaging system.

The findings of this study show the promising future potential of the developed field-imaging system for replacing subjective keystone and visual inspection methods or the time-consuming hand measurement process.

\subsection{SUMMARY OF FINDINGS FROM THE RIPRAP STOCKPILE STUDY}

Based on the findings of the riprap stockpile imaging study, the following conclusions can be drawn regarding the proposed stockpile aggregate image analysis approach:

- A comprehensive literature review showed that most existing aggregate imaging systems focused on separated or non-overlapping particles, yet an effective imaging approach for densely stacked or stockpile aggregates has not been well studied. No previous study was found on user-independent dense image-segmentation techniques for the characterization of aggregate material.

- This study adopted and successfully trained a neural network to accomplish the challenging stockpile aggregate image-segmentation task. By establishing an image dataset of labeled aggregate images, the trained segmentation model achieved good performance for extracting individual aggregate particles in an automated manner, thus greatly enhancing the efficiency of morphological analysis. This innovative research demonstrated the technology transfer and practical application of deep-learning techniques in stockpile aggregate imaging.

- Morphological analyses were conducted on the segmented aggregate particles to produce size and shape distribution curves. Analysis results were verified with ground-truth labeling to measure the robustness and accuracy of the segmentation approach.

- Completeness and precision analyses were conducted between the labeled images in the validation set and their segmentation results. The segmentation model exhibited precision and completeness in the stockpile image-segmentation task.

In summary, the deep-learning-based stockpile image-segmentation approach presented in this research paper is likely to advance state-of-the-art aggregate image analysis. Accordingly, this innovative technology is planned for full development to offer convenient and affordable means of quality control tasks conducted by state DOTs and aggregate producers, as well as provide field-scale 
material characterization methods for numerical simulations of granular material composition and packing.

\subsection{RECOMMENDATIONS FOR FUTURE WORK}

For the single-particle and riprap stockpile imaging studies, more advancements are envisioned and recommended for future research related to this topic. For example, the image training dataset can be further enriched to enhance the robustness and capability of the segmentation model. Because of the deep-learning nature, the model performance is likely to be scalable with increasing dataset size. For example, image data augmentation techniques (shift, flip, rotate, zoom, etc.) can be applied to the current dataset to artificially expand its size. Further, more stockpile aggregate images need to be collected and labeled for diverse geological origins and rock types (limestone, granite, sandstone, trap rock, etc.), with joint efforts by aggregate producers. Aggregate images containing various backgrounds can also be included in the database.

Moreover, the segmentation kernel presented herein could possibly serve as a versatile, generalpurpose image analysis engine for the existing and future developments of aggregate imaging systems. Upon establishing a comprehensive dataset, the kernel is likely to produce general background-independent segmentation of aggregate images. Based on the data-driven manner of deep-learning techniques, automated segmentation is expected to work under different conditions: (a) separated particles under laboratory-controlled lighting and background, (b) non-overlapping particles under natural lighting and random backgrounds, and (c) densely stacked particles in aggregate stockpiles. Hence, the setup of existing aggregate imaging systems may be greatly simplified, and the systems can be customized to have a wider range of use in research and industrial applications.

Further, intelligent methods of acquiring stockpile aggregate images can be integrated with advanced aerial photography techniques. For example, Unmanned Aircraft Systems (UAS) can greatly help with the image-acquisition step for multi-point or all-around inspection of a large stockpile, especially when intelligent route planning and attitude adjustment techniques are used.

Challenges and knowledge gaps, however, still exist for the advanced imaging techniques and their practical applications. More riprap materials from different size categories need to be studied to extend the scope of this research study. General-purpose imaging techniques will provide more benefit for tasks such as scene reconstruction using the Structure-from-Motion (SfM) approach, and single-view reconstruction based on RGB-Depth cameras. The major challenge of riprap stockpile evaluation originates from the fact that the particles in a riprap stockpile are only exposed with the particle surface. A significant amount of hidden information is in the unseen side of the particles as well as the central part of the stockpile. For the unseen side of the particles, more advanced 3D stockpile reconstruction techniques, such as those based on the point cloud technology tested in preliminary development during this research project, should be leveraged to acquire and compile as much shape information as possible. For the central part of the stockpile, the problem could be solved from a different perspective. Once the riprap particles are stacked into a stockpile, most of the size and shape information is hidden inside the stockpile with only surface information available. This 
indicates that more imaging techniques should be integrated into the entire production line of aggregate producers, such as during the crushing process, conveying process, etc. This also implies that there is great future potential and benefits of the joint efforts between the transportation agencies and end users of the aggregate imaging research and the aggregate production industry. 


\section{REFERENCES}

Alata, O., and L. Quintard. Is There a Best Color Space for Color Image Characterization or Representation Based on Multivariate Gaussian Mixture Model? Computer Vision and Image Understanding, Vol. 113, No. 8, 2009, pp. 867-877.

Al-Rousan, T., E. Masad, L. Myers, and C. Speigelman. New Methodology for Shape Classification of Aggregates. Transportation Research Record: Journal of the Transportation Research Board, No. 1913, 2005, pp. 11-23. https://doi.org/10.3141/1913-02.

Al-Rousan, T., E. Masad, E. Tutumluer, and T. Pan. Evaluation of Image Analysis Techniques for Quantifying Aggregate Shape Characteristics. Journal of Construction and Building Materials, Vol. 21, 2007, pp. 978-990.

Anochie-Boateng, J. K., J. Komba, and G. Mvelase. Three-Dimensional Laser Scanning Technique to Quantify Aggregate and Ballast Shape Properties. Journal of Construction and Building Materials, Vol. 43, 2013, pp. 389-398.

Arnab, A., and P. H. Torr. Pixelwise Instance Segmentation with a Dynamically Instantiated Network. Proc., IEEE Conference on Computer Vision and Pattern Recognition, Honolulu, Hawaii, 2017, pp. 441-450.

ASTM C127: Standard Test Method for Relative Density (Specific Gravity) and Absorption of Coarse Aggregate. ASTM International, West Conshohocken, PA, 2015.

ASTM D2940: Standard Specification for Graded Aggregate Material for Bases or Subbases for Highway or Airports. ASTM International, West Conshohocken, PA, 2015.

ASTM D448: Standard Classification for Size of Aggregate for Road and Bridge Construction. ASTM International, West Conshohocken, PA, 2017.

ASTM D5519: Standard Test Methods for Particle Size Analysis of Natural and Man-Made Riprap Materials. ASTM International, West Conshohocken, PA, 2015.

ASTM D6092: Standard Practice for Specifying Standard Sizes of Stone for Erosion Control. ASTM International, West Conshohocken, PA, 2014.

Barrett, P. J. The Shape of Rock Particles, A Critical Review. Sedimentology, Vol. 27, 1980.

Bessa, I. S., V. T. C. Branco, J. B. Soares, and J. A. N. Neto. Aggregate Shape Properties and Their Influence on the Behavior of Hot-Mix Asphalt. Journal of Materials in Civil Engineering, Vol. 27, No. 7, 2014, p.04014212.

Blodgett, J. C., and C. E. McConaughy. Water-Resources Investigations Report 86-4128: Rock Riprap Design for Protection of Stream Channels Near Highway Structures-Volume 2 Evaluation of Riprap Design Procedures. U.S. Geological Survey, Sacramento, 1986, pp. 9-18.

Bradley, D., and G. Roth. Adapting Thresholding Using the Integral Image. Journal of Graphics Tools, Vol. 12, No. 2, 2007, pp. 13-21.

Busin, L., N. Vandenbroucke, and L. Macaire. Color Spaces and Image Segmentation. In Advances in Imaging and Electron Physics, Vol. 151, Elsevier, New York, 2008, pp. 65-168. 
Chen, J. Discrete Element Method (DEM) Analyses for Hot-Mix Asphalt (HMA) Mixture Compaction. PhD dissertation. University of Tennessee at Knoxville, 2011.

Cheng, H. D., X. H. Jiang, Y. Sun, and J. Wang. Color Image Segmentation: Advances and Prospects. Pattern Recognition, Vol. 34, No. 12, 2001, pp. 2259-2281.

Chiew, Y. M. Mechanics of Riprap Failure at Bridge Piers. Journal of Hydraulic Engineering, Vol. 121, No. 9, 1995, pp. 635-643.

Christine, F. M. Advanced Color Image Processing and Analysis. Springer, New York, 2013.

Cremers, D., and K. Kolev. Multiview Stereo and Silhouette Consistency via Convex Functionals over Convex Domains. IEEE Transactions on Pattern Analysis and Machine Intelligence, Vol. 33, No. 6, 2011, pp. 1161-1174.

Dietterich, T. G., L. Pratt, and S. Thrun. Machine Learning-Special Issue on Inductive Transfer. Journal Machine Learning, Vol. 28, No. 1, 1997.

Dutta, A., and A. Zisserman. The VGG Image Annotator (VIA). arXiv preprint, 2019. https://arxiv.org/abs/1904.10699. Accessed July 1, 2019.

Eddie, B. The Software Development Process. August 2007, http://sce2.umkc.edu/BIT/burrise/pl/software-process/. Accessed September 22, 2019.

Feiler, Peter H., and Watts S. Humphrey. Software process development and enactment: Concepts and definitions. Proceedings of the Second International Conference on the Software ProcessContinuous Software Process Improvement, 1993.

Gates, L., E. Masad, R. Pyle, and D. Bushee. FHWA-HIF-11-030 Report: Aggregate Imaging Measurement System 2 (AIMS2). Highways for LIFE Program Office, Federal Highway Administration, Pine Instrument Company, 2011.

Ghauch, Z. Micromechanical Finite Element Modeling of Asphalt Concrete Materials Considering Moisture Presence. PhD dissertation. Department of Civil and Environmental Engineering, University of Illinois at Urbana-Champaign, 2014.

Gonzalez, R. C., and R. E. Woods. Digital Image Processing (3rd edition). Prentice Hall, New Jersey, 2006.

He, K., G. Gkioxari, P. Dollár, and R. Girshick. Mask R-CNN. Proc., IEEE International Conference on Computer Vision, Venice, Italy, 2017, pp. 2961-2969.

Hryciw, R. D., J. Zheng, H. S. Ohm, and J. Li. Innovations in Optical Geo-Characterization. In GeoCongress 2014 Keynote Lectures: Geo-Characterization and Modeling for Sustainability, 2014, pp. 97-116. https://doi.org/10.1061/9780784413289.005.

Huang, H. Discrete Element Modeling of Railroad Ballast Using Imaging Based Aggregate Morphology Characterization. PhD dissertation. Department of Civil and Environmental Engineering, University of Illinois at Urbana-Champaign, 2010.

Huang, H., J. Luo, M. Moaveni, E. Tutumluer, J. M. Hart, S. Beshears, and A. J. Stolba. Field Imaging and Volumetric Reconstruction of Riprap Rock and Large-Sized Aggregates: Algorithms and Application. Transportation Research Record: Journal of the Transportation Research Board, 2019. 
https://doi.org/10.1177/0361198119848704.

Huang, H., M. Moaveni, S. Schmidt, E. Tutumluer, and J. M. Hart. Evaluation of Railway Ballast Permeability Using Machine Vision-Based Degradation Analysis. Transportation Research Record: Journal of the Transportation Research Board, 2018. 2672: 62-73.

Illinois Department of Transportation (IDOT). Standard Specifications for Road and Bridge Construction. Illinois Department of Transportation, 2016, pp. 759-761.

Illinois Department of Transportation (IDOT). Policy Memorandum 14-08.2, July 2018.

http://www.idot.illinois.gov/Assets/uploads/files/Doing-Business/Manuals-Guides-\&Handbooks/Highways/Materials/Aggregate/14-08\%20erosion.pdf. Accessed July 2019.

Illinois Department of Transportation (IDOT). Manual of Test Procedures for Materials, December 2017. http://www.idot.illinois.gov/Assets/uploads/files/Doing-Business/Manuals-Guides-\&Handbooks/Highways/Materials/testproceduresmanual.pdf. Accessed July 2019.

Illinois Department of Transportation (IDOT). Approved/Qualified Producer List of Aggregate Sources, September 2018. http://www.idot.illinois.gov/Assets/uploads/files/Doing-Business/SpecialtyLists/Highways/Materials/Materials-\&-PhysicalResearch/Aggregate/approvedaggregatesources.pdf. Accessed July 2019.

Jin, C., X. Yang, Z. You, and K. Liu. Aggregate Shape Characterization Using Virtual Measurement of Three-Dimensional Solid Models Constructed from X-Ray CT Images of Aggregates. Journal of Materials in Civil Engineering, Vol. 30, No. 3, 2018, pp. 04018026. https://doi.org/10.1061/(ASCE)MT.1943-5533.0002210.

Kazmee, H., E. Tutumluer, and S. Beshears. Pavement Working Platforms Constructed with Large Size Unconventional Aggregates. Transportation Research Record: Journal of the Transportation Research Board, 2016.2578: 1-11.

Killick, R., P. Fearnhead, and I. A. Eckley. Optimal Detection of Change Points with a Linear Computational Cost. Journal of the American Statistical Association, Vol. 107, No. 500, 2012, pp. 1590-1598.

Komba, J., J. Anochie-Boateng, and W. van der Merwe Steyn. Analytical and Laser Scanning Techniques to Determine Shape Properties of Aggregates. Transportation Research Record: Journal of the Transportation Research Board, No. 2335, 2013, pp. 60-71. https://doi.org/10.3141/2335-07.

Kothari, R. What Are the Differences between RGB, HSV and CIE-Lab?, March 2018.

https://www.quora.com/What-are-the-differences-between-RGB-HSV-and-CIE-Lab. Accessed July 20, 2018.

Lagasse, P. F., P. E. Clopper, L. W. Zevenbergen, and J. F. Ruff. NCHRP Report 568: Riprap Design Criteria, Recommended Specifications, and Quality Control. Transportation Research Board, Washington, D.C., 2006.

Lagasse, P. F., L. W. Zevenbergen, J. D. Schall, and P. E. Clopper. FHWA Report NHI-01-003, Hydraulic Engineering Circular No. 23: Bridge Scour and Stream Instability Countermeasures. Federal Highway Administration, Washington, D.C., 2001. 
LeCun, Y., Y. Bengio, and G. Hinton. Deep Learning. Nature, Vol. 521, No. 7553, 2015, p.436.

Lin, T. Y., M. Maire, S. Belongie, J. Hays, P. Perona, D. Ramanan, P. Dollár, and C. L. Zitnick. Microsoft COCO: Common Objects in Context. Proc., European Conference on Computer Vision, Zurich, Switzerland, 2014, pp. 740-755.

Lippert, D. L. Illinois Department of Transportation. Inspection of Stone for Erosion Protection, Sediment Control, and Rockfill, June 2012.

http://www.idot.illinois.gov/Assets/uploads/files/Doing-Business/Manuals-Guides-\&Handbooks/Highways/Materials/Aggregate/14-08.1erosion.pdf. Accessed March 30, 2018.

Liu, Y., D. S. Lane, and W. Sun. No. FHWA/VTRC 19-R15: Influence of Aggregate Morphology and Grading on the Performance of 9.5-mm Stone Matrix Asphalt Mixtures. Federal Highway Administration, 2019.

Long, J., E. Shelhamer, and T. Darrell. Fully Convolutional Networks for Semantic Segmentation. Proc., IEEE Conference on Computer Vision and Pattern Recognition, Boston, Massachusetts, 2015, pp. 3431-3440.

Lutton, R. J., B. J. Houston, and J. B. Warriner. No. WES/TR/GL-81-8: Evaluation of Quality and Performance of Stone as Riprap or Armor. Army Engineer Waterways Experiment Station Vicksburg MS Geotechnical Lab, 1981.

Maerz, N. H., and T. C. Palangio. WipFrag System II-Online Fragmentation Analysis. Proceedings of the FRAGBLAST 6 Sixth International Symposium for Rock Fragmentation by Blasting, Johannesburg, South Africa, 1999, pp. 111-115.

Maerz, N. H., T. C. Palangio, and J. A. Franklin. WipFrag Image Based Granulometry System. Proceedings of the FRAGBLAST 5 Workshop on Measurement of Blast Fragmentation, Montreal, Quebec, Canada, 1996, pp. 91-99.

Masad, E. NCHRP-IDEA Project 77 Report: The Development of a Computer Controlled Image Analysis System for Measuring Aggregate Shape Properties. Transportation Research Board, Washington, D.C., 2003.

Masad, E., T. Al-Rousan, M. Bathina, J. McGahan, and C. Spiegelman. Analysis of Aggregate Shape Characteristics and its Relationship to Hot Mix Asphalt Performance. International Journal of Road Materials and Pavement Design, Vol. 8, No. 2, 2007, pp. 317-350.

Moaveni, M., S. Wang, J. M. Hart, E. Tutumluer, and N. Ahuja. Evaluation of Aggregate Size and Shape by Means of Segmentation Techniques and Aggregate Image Processing Algorithms.

Transportation Research Record: Journal of the Transportation Research Board, No. 2335, 2013, pp. 50-59. https://doi.org/10.3141/2335-06.

Obaidat, M. T., K. A. Ghuzlan, and M. M. Alawneh. Analysis of Volumetric Properties of Bituminous Mixtures Using Cellular Phones and Image Processing Techniques. Canadian Journal of Civil Engineering, Vol. 44, No. 9, 2017, pp. 715-726.

Otsu, N. A. Threshold Selection Method from Gray-Level Histograms. IEEE Transactions on Systems, Man, and Cybernetics, Vol. 9, No. 1, 1979, pp. 62-66. 
Pan, T., E. Tutumluer, and J. Anochie-Boateng. Aggregate Morphology Affecting Resilient Behavior of Unbound Granular Materials. Transportation Research Record: Journal of Transportation Research Record, No. 1952, 2006, pp. 12-20. https://doi.org/10.3141/1952-02.

Polat, R., M. M. Yadollahi, A. E. Sagsoz, and S. Arasan. The Correlation between Aggregate Shape and Compressive Strength of Concrete: Digital Image Processing Approach. International Journal of Structure and Civil Engineering Research, Vol. 2, No. 3, 2013, pp. 62-80.

Prince, S. J. Computer Vision: Models, Learning, and Inference. Cambridge University Press, New York, 2012.

Pusuluri, N. R. Software Testing Concepts and Tools. Dreamtech Press, 2006.

Qamhia, I., E. Tutumluer, and H. Ozer. FHWA-ICT-18-016 Report: Field Performance Evaluation of Sustainable Aggregate By-product Applications. Illinois Center for Transportation/Illinois Department of Transportation, 2018.

Qian, Y. Integrated Computational and Experimental Framework for the Assessment of Railroad Ballast Life-Cycle Behavior. PhD dissertation. Department of Civil and Environmental Engineering, University of Illinois at Urbana-Champaign, 2015.

Quiroga, P. N., and D. W. Fowler. ICAR Research Report No. 104-1F: The Effects of Aggregate Characteristics on the Performance of Portland Cement Concrete. International Center for Aggregates Research, 2004.

Rao, C. Development of 3-D Image Analysis Techniques to Determine Shape and Size Properties of Coarse Aggregate. PhD dissertation. Department of Civil and Environmental Engineering, University of Illinois at Urbana-Champaign, 2001.

Rao, C., E. Tutumluer, and I.T. Kim. Quantification of Coarse Aggregate Angularity based on Image Analysis. Transportation Research Record: Journal of the Transportation Research Board, No. 1787, 2002, pp. 117-124. https://doi.org/10.3141/1787-13.

Rehman, R. U., and C. Paul. The Linux Development Platform: Configuring, Using, and Maintaining a Complete Programming Environment. Prentice Hall Professional, 2003.

Richardson, E. V., and S. R. Davis. FHWA Report NHI-01-001, Hydraulic Engineering Circular No. 18: Evaluating Scour at Bridges. Federal Highway Administration, Washington, D.C., 2001.

Romera-Paredes, B., and P. H. S. Torr. Recurrent Instance Segmentation. Proc., European Conference on Computer Vision, Amsterdam, The Netherlands, 2016, pp. 321-329.

Senthilkumaran, N., and R. Rajesh. Edge Detection Techniques for Image Segmentation -A Survey of Soft Computing Approaches. International Journal of Recent Trends in Engineering, Vol. 1, No. 2, 2009, p.250.

Sillick, S. Montana Department of Transportation. AASHTO RAC Member Survey Results, July 2017. https://research.transportation.org/rac-survey-detail/?survey id=371. Accessed July 20, 2018.

Tutumluer, E., N. Ahuja, J. M. Hart, M. Moaveni, H. Huang, Z. Zhao, and S. Shah. Report No. Safety-27: Field Evaluation of Ballast Fouling Conditions Using Machine Vision. Transportation Research Board, Washington, D.C., 2017. 
Tutumluer, E., and T. Pan. Aggregate Morphology Affecting Strength and Permanent Deformation Behavior of Unbound Aggregate Materials. Journal of Materials in Civil Engineering, Vol. 20, No. 9, 2008, pp. 617-627.

Tutumluer, E., C. Rao, and J. A. Stefanski. FHWA-IL-UI-278 Report: Video Image Analysis of Aggregates. University of Illinois Urbana-Champaign, Urbana, Illinois, 2000.

Vincent, L., and P. Soille. Watershed in Digital Spaces: An Efficient Algorithm Based on Immersion Simulation. IEEE Transactions on Pattern Analysis and Machine Intelligence, Vol. 13, No. 6, 1991, pp. 583-598.

Wang, L., W. Sun, E. Tutumluer, and C. Druta. Evaluation of Aggregate Imaging Techniques for Quantification of Morphological Characteristics. Transportation Research Record: Journal of the Transportation Research Board, No. 2335, 2013, pp. 39-49. https://doi.org/10.3141/2335-05.

Wang, X., R. Hansch, L. Ma, and O. Hellwich. Comparison of Different Color Spaces for Image Segmentation Using Graph-Cut. Proceedings of 2014 International Conference on Computer Vision Theory and Applications, Lisbon, Portugal, 2014, pp. 301-308.

Wani, M. A., and B. G. Batchelor. Edge-Region-Based Segmentation of Range Images. IEEE Transactions on Pattern Analysis and Machine Intelligence, Vol. 16, 1994, pp.314-319.

Wnek, M. A., E. Tutumluer, M. Moaveni, and E. Gehringer. Investigation of Aggregate Properties Influencing Railroad Ballast Performance. Transportation Research Record: Journal of Transportation Research Board, 2013. 2374, 180-190.

Zhao, Z. Q., P. Zheng, S. T. Xu, and X. Wu. Object Detection with Deep Learning: A Review. IEEE Transactions on Neural Networks and Learning Systems, 2019, pp. 1-21.

Zheng, J., and R. D. Hryciw. Identification and Characterization of Particle Shapes from Images of Sand Assemblies Using Pattern Recognition. Journal of Computing in Civil Engineering, Vol. 32, No. 3, 2018, p.04018016.

Zheng, J., and R. D. Hryciw. Soil Particle Size and Shape Distributions by Stereophotography and Image Analysis. Geotechnical Testing Journal, Vol. 40, No. 2, 2017, pp. 317-328. http://dx.doi.org/10.1520/ GTJ20160165. ISSN 0149-6115.

Zheng, J., and R. D. Hryciw. Soil Particle Size Characterization by Stereo-photography. In Geo-Congress 2014: Geo-characterization and Modeling for Sustainability, 2014, pp. 64-73. https://doi.org/10.1061/9780784413272.007. 


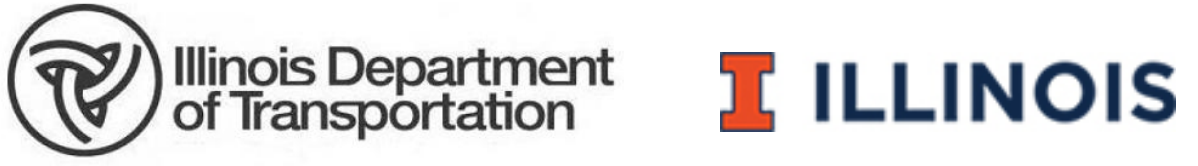

